\title{
The contributions of the Genome Project to the study of schistosomiasis
}

\author{
Adhemar Zerlotini, Guilherme Oliveira/ ${ }^{+}$ \\ Instituto de Pesquisa René Rachou-Fiocruz, Laboratório de Parasitologia Celular e Molecular, \\ Centro de Excelência em Bioinformática, Av. Augusto de Lima 1715, 30190-002 Belo Horizonte, MG, Brasil
}

In this paper we review the impact that the availability of the Schistosoma mansoni genome sequence and annotation has had on schistosomiasis research. Easy access to the genomic information is important and several types of data are currently being integrated, such as proteomics, microarray and polymorphic loci. Access to the genome annotation and powerful means of extracting information are major resources to the research community.

Key words: Schistosoma mansoni - genome - database - data integration

Genome sequencing technologies have considerably expanded our range of tools for experimental and theoretical approaches in the quest for understanding the molecular aspects of schistosomiasis and the design of new control tools.

The Schistosoma mansoni genome sequence contains over 360 million base pairs divided into seven pairs of autosomes and one pair of sex chromosomes (female $=\mathrm{ZW}$, male $=\mathrm{ZZ})($ Berriman et al. 2009).

The Wellcome Trust Sanger Institute and an international group of researchers have provided the genome sequencing assembly and annotation (Berriman et al. 2009). The latest draft version of the assembly (Release $4.0)$ is available online as contigs $(50,376)$ or supercontigs/scaffolds $(19,022)$. Almost half of the genome $(45 \%)$ was found to be composed of repetitive elements.

Both $a b$ initio and evidence based algorithms were used to perform gene prediction and the final automatically annotated sequence includes 11,809 protein-coding gene structures and 13,197 transcripts. It is worth noting that two major Brazilian transcriptome sequencing efforts provided large amounts of expressed sequence tags (EST) (Verjovski-Almeida et al. 2003, Oliveira et al. 2008) that were of critical importance for the identification of the coding regions in the genome. EST data can also be further used for the investigation of transcript variations such as in differential splicing (DeMarco et al. 2006) and alternative polyadenylation (Tian et al. 2007). To infer gene function, several computational analyses were performed using Basic Local Alignment Search Tool (BLAST) (Altschul et al. 1997) for similar-

Financial support: Fogarty International Center (5D43TW00701204), FAPEMIG (CBB-1181/0, REDE-186/08, 5323-4.01/07)

+Corresponding author: oliveira@cpqrr.fiocruz.br

Received 8 January 2009

Accepted 4 September 2009 ity searches, Gene Ontology (Harris et al. 2004) and InterPro (Mulder \& Apweiler 2008) for protein domain assignments and limited manual annotation.

SchistoDB: S. mansoni genome database - To establish a central repository for $S$. mansoni genomic data, a database, SchistoDB (Zerlotini et al. 2009) was developed. Similar to other parasite databases with the same architecture (Genomics Unified Schema (Davidson et al. 2001) such as PlasmoDB (Aurrecoechea et al. 2009), ToxoDB (Gajria et al. 2008) and CryptoDB (Heiges et al. 2006), the $S$. mansoni database provides the community wide access to the latest genome sequence, annotation and other types of data integrated with the genome information.

The genome data is structured in a robust relational database coupled with a powerful querying system so that searches can be combined to filter the information based on several criteria. The genome sequences were computationally reanalysed and integrated into a number of public genomic resources.

SchistoDB currently provides over 30 different queries and tools for analysis, retrieving or viewing the data. Users can integrate different search results using the "Query History" page, refining the original query iteratively, until a narrow list of genes of interest is obtained. The data can be downloaded in a flat file format for further analysis and each gene possesses its own record page that contains detailed information of all performed analyses (Supplementary data). GBrowse genome browser is used to display gene models, EST alignments, BLAST results, protein features etc and facilitates downloading data in various formats.

Genomic data analysis - Orthology information provided by the OrthoMCL group (Chen et al. 2006) has been integrated into SchistoDB. In this database orthologous genes from 87 species are clustered based on sequence similarity. The immediate result is the ability to infer protein function through evolutionary relationships, since orthologous genes diverged from a common ancestor owing to speciation events. Additionally orthology information allows us to directly compare $S$. mansoni genes to other species to narrow a list of candidate drug targets, for example. 
Using the complete annotated gene set, it is possible to predict the organism's metabolic pathways and gain insight into the physiology of S. mansoni. SchistoDB contains metabolic pathway prediction including approximately 607 enzymatic reactions and 112 pathways that were inferred to occur in the organism based on genome annotation and sequence similarity searches. This information can be used to extend the genome annotation and to compare $S$. mansoni with other organisms.

Several tegumental proteins have been identified as potential vaccine candidates (van Balkom et al. 2005, Braschi et al. 2006b) using proteomic approaches. Such research will benefit from the predicted proteome, not only because it enables the identification of mass fingerprints and peptides, but also because these sequences are computationally characterised to have transmembrane motifs or signal peptides and other types of annotation.

Next generation sequencing technologies have become available to $S$. mansoni research groups, allowing the generation of an extremely large sequence data set in each run. Thus, mapping transcript sequences to the genome, for example, will substantially assist intron/ exon boundary validation, thereby improving the gene models and genome assembly. Transcript sequences are also invaluable for alternative splicing, single nucleotide polymorphisms and indel studies.

Post-genomic analysis using primarily proteomic and microarray methods is currently being explored by several groups. These experimental approaches, enabled by the genome sequence, have produced essential contributions to a global understanding of how the parasites display sexual differentiation (Waisberg et al. 2008), adapt during development (Jolly et al. 2007) and, for example, how protein expression is compartmentalised (Braschi et al. 2006a). However, these data need to be fully integrated with the genome data to enable the community to make the most use of it.

One remaining challenge is identifying the function of the over $40 \%$ of unannotated sequences in the genome. Transgenesis and gene silencing by knockout or knockdown experiments will be essential in that process. These technologies remain largely unavailable. However, recent advances were made with the use of RNA interference (Geldhof et al. 2007, Ndegwa et al. 2007). These methods, in combination with the genomic data, will permit a more profound understanding of the biology of schistosomes and undoubtedly the design of new control measures.

Genome sequencing and annotation has impacted how molecular research is conducted in schistosomes. Issues related to data sharing and data standards still need to be fully resolved. However, the organisation of the information and the availability of robust querying tools, enabled by a relational genome database such as SchistoDB (http://www.schistodb.net), have provided a framework that provides faster access to the information and empowers groups that are not equipped to conduct the required computational analysis to make use of the information.

\section{REFERENCES}

Altschul SF, Madden TL, Schäffer AA, Zhang J, Zhang Z, Miller W, Lipman DJ 1997. Gapped BLAST and PSI-BLAST: a new generation of protein database search programs. Nucleic Acids Res 25: 3389-3402.

Aurrecoechea C, Brestelli J, Brunk BP, Dommer J, Fischer S, Gajria B, Gao X, Gingle A, Grant G, Harb OS, Heiges M, Innamorato F, Iodice J, Kissinger JC, Kraemer E, Li W, Miller JA, Nayak V, Pennington C, Pinney DF, Roos DS, Ross C, Stoeckert CJ Jr 2009. PlasmoDB: a functional genomic database for malaria parasites. Nucleic Acids Res 37: D539-543.

Berriman M, Haas BJ, LoVerde PT, Wilson RA, Dillon GP, Cerqueira GC, Mashiyama ST, Al-Lazikani B, Andrade LF, Ashton PD, Aslett MA, Bartholomeu DC, Blandin G, Caffrey CR, Coghlan A, Coulson R, Day TA, Delcher A, DeMarco R, Djikeng A, Eyre T, Gamble JA, Ghedin E, Gu Y, Hertz-Fowler C, Hirai H, Hirai Y, Houston R, Ivens A, Johnston DA, Lacerda D, Macedo CD, McVeigh P, Ning Z, Oliveira G, Overington JP, Parkhill J, Pertea M, Pierce RJ, Protasio AV, Quail MA, Rajandream MA, Rogers J, Sajid M, Salzberg SL, Stanke M, Tivey AR, White O, Williams DL, Wortman J, Wu W, Zamanian M, Zerlotini A, Fraser-Liggett CM, Barrell BG, El-Sayed NM 2009. The genome of the blood fluke Schistosoma mansoni. Nature 460: 352-358.

Braschi S, Borges WC, Wilson RA 2006a. Proteomic analysis of the schistosome tegument and its surface membranes. Mem Inst Oswaldo Cruz 101 (Suppl. I): 205-212.

Braschi S, Curwen RS, Ashton PD, Verjovski-Almeida S, Wilson A 2006b. The tegument surface membranes of the human blood parasite Schistosoma mansoni: a proteomic analysis after differential extraction. Proteomics 6: 1471-1482.

Chen F, Mackey AJ, Stoeckert CJ Jr, Roos DS 2006. OrthoMCL-DB: querying a comprehensive multi-species collection of ortholog groups. Nucleic Acids Res 34: D363-368.

Davidson SB, Crabtree J, Brunk B, Schug J, Tannen V, Overton GC, Stoeckert CJ Jr 2001. K2/Kleisli and GUS: experiments in integrated access to genomic data sources. IBM Systems J 40: 512-531.

DeMarco R, Oliveira KC, Venancio TM, Verjovski-Almeida S 2006. Gender biased differential alternative splicing patterns of the transcriptional cofactor CA150 gene in Schistosoma mansoni. Mol Biochem Parasitol 150: 123-131.

Gajria B, Bahl A, Brestelli J, Dommer J, Fischer S, Gao X, Heiges M, Iodice J, Kissinger JC, Mackey AJ, Pinney DF, Roos DS, Stoeckert CJJr, Wang H, Brunk BP 2008. ToxoDB: an integrated Toxoplasma gondii database resource. Nucleic Acids Res 36: D553-556.

Geldhof P, Visser A, Clark D, Saunders G, Britton C, Gilleard J, Berriman M, Knox D 2007. RNA interference in parasitic helminths: current situation, potential pitfalls and future prospects. Parasitology 134: 609-619.

Harris MA, Clark J, Ireland A, Lomax J, Ashburner M, Foulger R, Eilbeck K, Lewis S, Marshall B, Mungall C, Richter J, Rubin GM, Blake JA, Bult C, Dolan M, Drabkin H, Eppig JT, Hill DP, Ni L, Ringwald M, Balakrishnan R, Cherry JM, Christie KR, Costanzo MC, Dwight SS, Engel S, Fisk DG, Hirschman JE, Hong EL, Nash RS, Sethuraman A, Theesfeld CL, Botstein D, Dolinski K, Feierbach B, Berardini T, Mundodi S, Rhee SY, Apweiler R, Barrell D, Camon E, Dimmer E, Lee V, Chisholm R, Gaudet P, Kibbe W, Kishore R, Schwarz EM, Sternberg P, Gwinn M, Hannick L, Wortman J, Berriman M, Wood V, de la Cruz N, Tonellato P, Jaiswal P, Seigfried T, White R, Gene Ontology Consortium 2004. The Gene Ontology (GO) database and informatics resource. Nucleic Acids Res 32: D258-261. 
Heiges M, Wang H, Robinson E, Aurrecoechea C, Gao X, Kaluskar N, Rhodes P, Wang S, He CZ, Su Y, Miller J, Kraemer E, Kissinger JC 2006. CryptoDB: a Cryptosporidium bioinformatics resource update. Nucleic Acids Res 34: D419-422.

Jolly ER, Chin CS, Miller S, Bahgat MM, Lim KC, De Risi J, McKerrow JH 2007. Gene expression patterns during adaptation of a helminth parasite to different environmental niches. Genome Biol 8: R65.

Mulder NJ, Apweiler R 2008. The InterPro database and tools for protein domain analysis. In Current Protocols Bioinformatics, chapter 2, unit 2.7, John Wiley \& Sons, New Jersey.

Ndegwa D, Krautz-Peterson G, Skelly PJ 2007. Protocols for gene silencing in schistosomes. Exp Parasitol 117: 284-291.

Oliveira G, Franco G, Verjovski-Almeida S 2008. The Brazilian contribution to the study of the Schistosoma mansoni transcriptome. Acta Trop 108: 179-182.

Tian B, Pan Z, Lee JY 2007. Widespread mRNA polyadenylation events in introns indicate dynamic interplay between polyadenylation and splicing. Genome Res 17: 156-165. van Balkom BW, van Gestel RA, Brouwers JF, Krijgsveld J, Tielens AG, Heck AJ, van Hellemond JJ 2005. Mass spectrometric analysis of the Schistosoma mansoni tegumental sub-proteome. J Proteome Res 4: 958-966.

Verjovski-Almeida S, DeMarco R, Martins EA, Guimarães PE, Ojopi EP, Paquola AC, Piazza JP, Nishiyama MY Jr, Kitajima JP, Adamson RE, Ashton PD, Bonaldo MF, Coulson PS, Dillon GP, Farias LP, Gregorio SP, Ho PL, Leite RA, Malaquias LC, Marques RC, Miyasato PA, Nascimento AL, Ohlweiler FP, Reis EM, Ribeiro MA, Sá RG, Stukart GC, Soares MB, Gargioni C, Kawano T, Rodrigues V, Madeira AM, Wilson RA, Menck CF, Setubal JC, Leite LC, Dias-Neto E 2003. Transcriptome analysis of the acoelomate human parasite Schistosoma mansoni. Nat Genet 35: 148-157.

Waisberg M, Lobo FP, Cerqueira GC, Passos LK, Carvalho OS, ElSayed NM, Franco GR 2008. Schistosoma mansoni: microarray analysis of gene expression induced by host sex. Exp Parasitol 120: $357-363$.

Zerlotini A, Heiges M, Wang H, Moraes RL, Dominitini AJ, Ruiz JC, Kissinger JC, Oliveira G 2009. SchistoDB: a Schistosoma mansoni genome resource. Nucleic Acids Res 37: D579-582. 


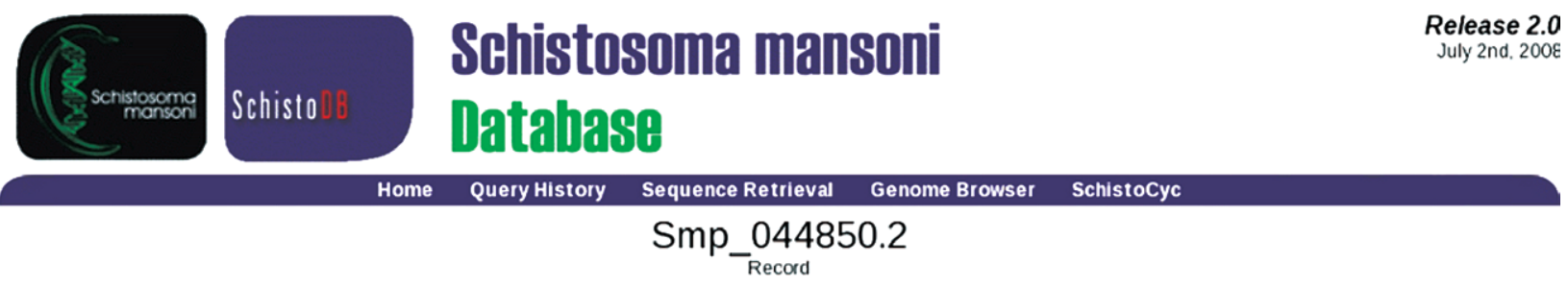

Overview

This Schistosoma mansoni gene spans positions 261868 - 266439 of contig Smp_scaff000112.

Approximate protein mol. wh. (Daltons): 39147 [computed from tav transtatan]

proauct

ribokinase. putative

Links to Other Web Pages

SchistoCyc Metabolic Pathway Database

Sanger GeneDB

Genomic Contex

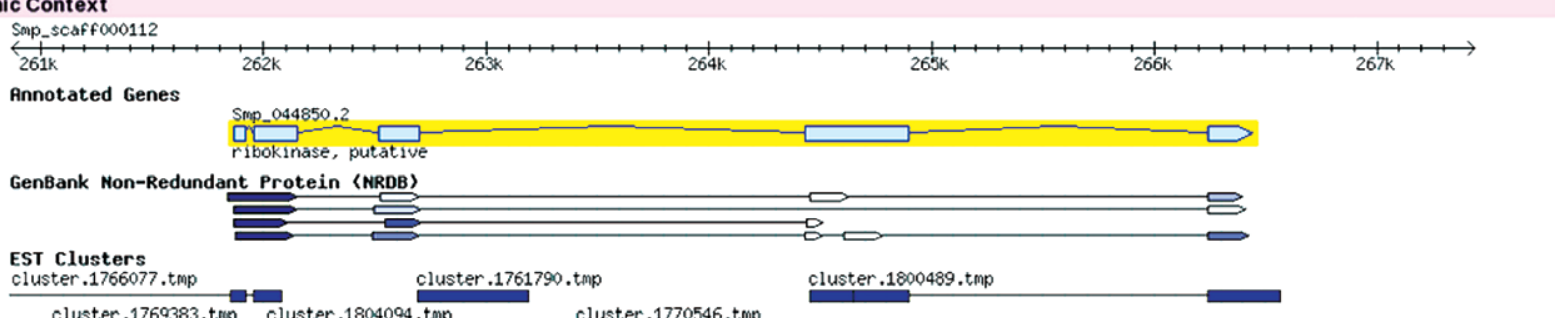

$\underset{\text { cluster.1769383.tmp cluster.1804094.tmp cluster.1761790.tmp }}{\text { cluster.1770546.tmp }}$

cluster.1804092.tmp

cluster.1804093.tmp

cluster.1804091.tmp

Ver jouski oligos

Q2_P08648

$\underset{\text { Q2_P25385 } P 06350}{\text { Q2 _P25887 }}$

ORFgt50

View in Genome Bronser

\section{EC Numbers}

Accession

2.71 .15

2.7 .11 .24

Description

Ribokinase

Mitogen-activated protein kinase

Go Terms

Ontology

F

p

GO ID

GO:0004747

Go Term Name

ribokinase activity

D-ribose metabolic process

GO:0006014
Q2_P19222

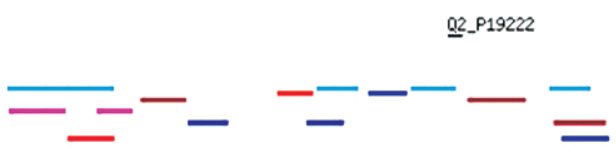

Kegg Drugs

Entry Name

D03736 Doramapimod

Synonyms Activity

CAS PubChem

Treatment of theumatoid arthrits. Crohn's disease and psoriasis

$\begin{array}{ll}\text { Source } & \text { Evidence Code } \\ \text { Interpro } & \text { IEA } \\ \text { Interpro } & \text { IEA }\end{array}$

Schistosoma mansoni Paralogs(OrthoMCL DB)

none

Find Smp_044850.2 in OrthoMCL DB

\section{Predicted Protein Features}

Smp_044850.2-1

$1020 \quad 3040 \quad 50 \quad 6070 \quad 8090100110120130140150160170180190200210220230240250260270280290300310320330340350360$

Kyte-Doolittle Hydropathy Plot

(Kyte-00olittle hydropathy plot

Interpro Donains

SSF53613 0043278

PF00294 PfKB

PROOG9O RIBOKINASE

PROO990 RIBOKINASE

PR00990 RIBOKINASE

PRO0990 RIBOKINASE

Signal Peptide 
Transnenbrane Donains

\section{BLASTP Hits}

gi|147899756|ref|NP_001088727.1| hypothetical protein LOC495991 [Xenopus laevis]• gi|109102478|ref|XP_001100564.1| PREOICTED: similar to ribokinase isoform 2 [Macaca mulatta]

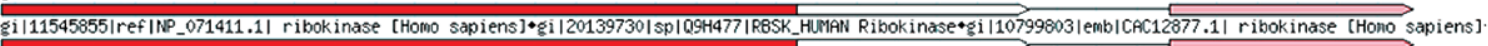
gi|33304089|gb|AAQ02552.1| ribokinase [synthet ic construct]

gi| 56757009 |gb|ARt26676.1/ SJCHGC06996 protein [Schistoscona japonicum]

\begin{tabular}{|c|c|}
\hline \multicolumn{2}{|c|}{ Similarities to Protein Data Bank (PDB) Chains } \\
\hline PDB Structure & PDB Description \\
\hline 2fV7_A & Ribokinase \\
\hline 2fv7_B & Ribokinase \\
\hline 1gqt_C & RIBOKINASE \\
\hline 1gqt_D & RIBOKINASE \\
\hline 1rk2_A & RIBOKINASE \\
\hline 1rk2_B & RIBOKINASE \\
\hline 1rk2_C & RIBOKINASE \\
\hline 1gqt_B & RIBOKINASE \\
\hline 1gqt_A & RIBOKINASE \\
\hline 1rk2_D & RIBOKINASE \\
\hline 1rks_A & PROTEIN (RIBOKINASE \\
\hline $1 \mathrm{rkd}$ & RIBOKINASE \\
\hline 1rka_A & PROTEIN (RIBOKINASE \\
\hline 1vm7_A & ribokinase \\
\hline 1vm7_B & ribokinase \\
\hline 1 tyy_A & putative sugar kinase \\
\hline 1tz3_B & putative sugar kinase \\
\hline 1tz3_A & putative sugar kinase \\
\hline 1 tyy_B & putative sugar kinase \\
\hline $1 \mathrm{tz} 6 \_\mathrm{A}$ & putative sugar kinase \\
\hline
\end{tabular}

\section{Taxon}

Homo sapiens

Homo sapiens

Escherichia coli

Escherichia coli

Escherichia coli

Escherichia coli

Escherichia coli

Escherichia coli

Escherichia coli

Escherichia coli

Escherichia coli

Escherichia coli

Escherichia coli

Thermotoga maritima MSB8

Thermotoga maritima MSB8

Salmonella typhimurium $L T 2$

Salmonella typhimurium LT2

Salmonella typhimurium LT2

Salmonella typhimurium LT2

Salmonella typhimurium LT2

\% Coverage
92
92
71
71
71
71
71
71
71
71
71
71
71
78
78
62
62
62
62
62

\section{\% Identity}

42

42

34

34

34

34

34

34

34

34

34

34

34

33

33

32

32

32

32
32

32

\section{P.value}

$1.3 \times 10^{-53}$

$1.3 \times 10^{-53}$

$5.2 \times 10^{-26}$

$5.2 \times 10^{-26}$

$52 \times 10^{-26}$

$5.2 \times 10^{-26}$

$5.2 \times 10^{-26}$

$5.2 \times 10^{-26}$

$52 \times 10^{-26}$

$52 \times 10^{-26}$

$5.2 \times 10^{-26}$

$5.2 \times 10^{-26}$

$5.2 \times 10^{-26}$

$4.7 \times 10^{-18}$

$47 \times 10^{-18}$

$2.7 \times 10^{-8}$

$2.7 \times 10^{-\varepsilon}$

$2.7 \times 10^{-\varepsilon}$

$2.7 \times 10^{-\varepsilon}$

$2.7 \times 10^{-\varepsilon}$

Predicted Protein Sequence

MKKSHVTVVGSLNDLSVTTNH IPKVCETVTGSAT LLGYGGKGANDCVASTVLGCNTALV

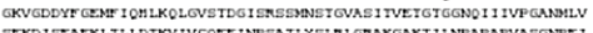

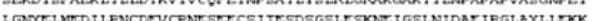

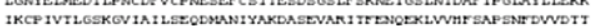

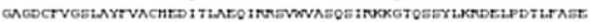

TrCosp

Sequence Length. 365 aa

Genome Sequencing and Annotation by:

Preliminary sequence data for S. mansoni genome was obtained from GeneDB website.

Haas BJ, Berriman M, Hirai H, Cerqueira GG, Loverde PT, EI-Sayed NM. Schistosoma mansoni genome: closing in on a final gene set. Exp Parasitol. 2007 Nov, 117(3) 225-8.

El-Sayed NM. Bartholomeu D. Ivens A, Johnston DA, LoVerde PT. Advances in schistosome genomics. Trends Parasitol. 2004 Apr: 20 (4):154-7.

\begin{tabular}{|ccc}
\hline SchistoDB & Schistosoma mansoni genome resource contact \\
Date: $07 / 2008$ & & For help con \\
\hline
\end{tabular}

Screenshot from SchistoDB displaying the gene record page. In the example, the gene for ribokinase is displayed. 
TABLE

Results of specific exams of 27 patients with presumptive diagnosis of schistosomal myeloradiculopathy examined in hospitals of Campinas, São Paulo (SP), Brazil between 1995-2005

\begin{tabular}{|c|c|c|c|c|c|c|c|}
\hline \multirow[b]{2}{*}{ Patient } & \multirow[b]{2}{*}{$\begin{array}{l}\text { Local where } \\
\text { infection } \\
\text { probably occurred }\end{array}$} & \multirow[b]{2}{*}{$\begin{array}{l}\text { Time of } \\
\text { follow-up }\end{array}$} & \multicolumn{5}{|c|}{ Specific examinations } \\
\hline & & & $\begin{array}{c}\text { Stool } \\
\text { examination }\end{array}$ & $\begin{array}{c}\text { Number of } \\
\text { samples }\end{array}$ & $\begin{array}{l}\text { Immune } \\
\text { reaction } \\
\text { in serum }\end{array}$ & $\begin{array}{l}\text { Immune } \\
\text { reaction } \\
\text { in CSF }\end{array}$ & $\begin{array}{c}\text { Other } \\
\text { examinations }\end{array}$ \\
\hline 1 & Campinas (SP) & 6 years & + & 4 & + & ND & $\begin{array}{c}\text { Kato-Katz } \\
\text { (8 EPG) }\end{array}$ \\
\hline 2 & Campinas (SP) & 8 years & + & 1 & ND & ND & $\begin{array}{l}\text { Kato-Katz } \\
\text { (48 EPG) }\end{array}$ \\
\hline 3 & Amparo (SP) & 6 years & - & 1 & ND & + & - \\
\hline 4 & Campinas (SP) & 5 years & - & 3 & + & ND & $\begin{array}{c}\text { Rectal mucosa } \\
\text { biopsy negative } \\
\text { for Schistosoma } \\
\text { mansoni }\end{array}$ \\
\hline 5 & Campinas (SP) & 4 years & + & 1 & ND & + & - \\
\hline 6 & Limeira (SP) & 2 years & ND & ND & ND & ND & $\begin{array}{l}\text { Positive to spinal } \\
\text { cord biopsy }\end{array}$ \\
\hline 7 & Campinas (SP) & 3 months & - & 2 & ND & + & - \\
\hline 8 & Campinas (SP) & 6 years 3 months & + & 2 & ND & ND & - \\
\hline 9 & Campinas (SP) & 5 years 8 months & + & 3 & ND & ND & $\begin{array}{l}\text { Positive to spinal } \\
\text { cord biopsy }\end{array}$ \\
\hline 10 & Campinas (SP) & 7 years 5 months & - & 1 & + & + & - \\
\hline 11 & Campinas (SP) & 10 years & - & 3 & ND & + & $\begin{array}{l}\text { Positive rectal } \\
\text { mucosa biopsy }\end{array}$ \\
\hline 12 & Campinas (SP) & 4 years & - & 1 & ND & + & - \\
\hline 13 & Limeira (SP) & 5 years 6 months & Ignored & Ignored & ND & + & - \\
\hline 14 & Campinas (SP) & 2 months & + & 2 & + & + & - \\
\hline 15 & Minas Gerais (MG) & 1 year & + & 1 & + & - & $\begin{array}{c}\text { Kato-Katz } \\
\text { (8 EPG) }\end{array}$ \\
\hline 16 & Sergipe & 2 months & + & 2 & ND & + & - \\
\hline 17 & Nova Módica (MG) & 1 year 3 months & - & 2 & ND & + & - \\
\hline 18 & Porteirinha (MG) & 3 years & - & 3 & ND & + & - \\
\hline 19 & MG & 1 year 5 months & + & 2 & ND & + & - \\
\hline 20 & Guanambi (BA) & 1 year 4 months & - & 3 & + & ND & - \\
\hline 21 & MG & 2 years 2 months & - & 2 & ND & + & - \\
\hline 22 & No information & 10 days & + & 1 & ND & ND & - \\
\hline 23 & No information & 6 years & - & 1 & ND & + & - \\
\hline 24 & Caratinga (MG) & 6 years & + & 2 & ND & ND & $\begin{array}{c}\text { Kato-Katz } \\
\text { (19 EPG) }\end{array}$ \\
\hline 25 & Alagoas & 1 year & - & 1 & - & + & - \\
\hline 26 & Curvelo (MG) & 12 years & - & 3 & ND & ND & $\begin{array}{l}\text { Positive rectal } \\
\text { mucosa biopsy }\end{array}$ \\
\hline 27 & Lajinha (MG) & 4 years & - & 1 & + & ND & - \\
\hline
\end{tabular}

EPG: eggs per gram; CSF: cerebrospinal fluid; ND: not done. 


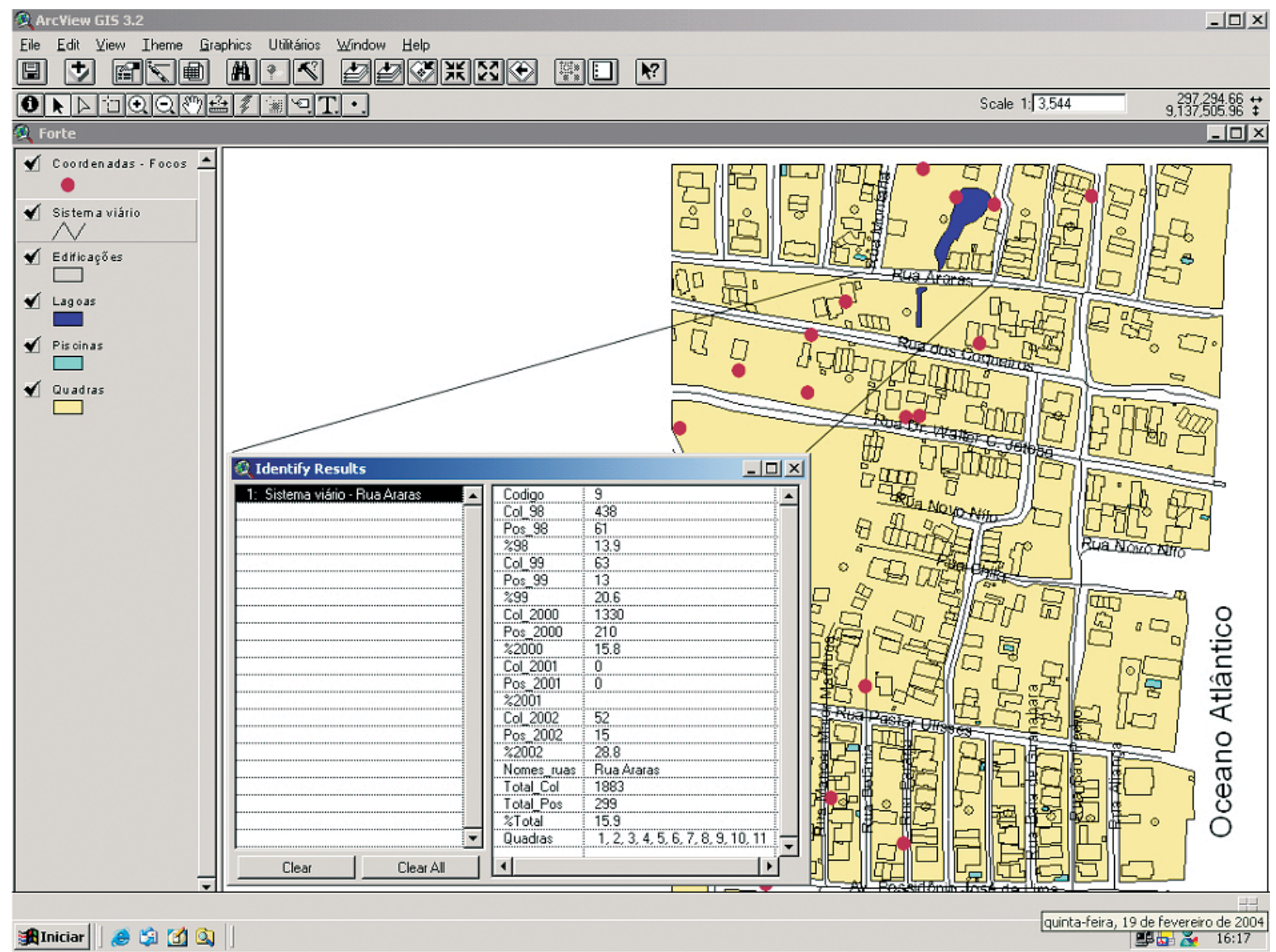

Fig. 5: application for monitoring the foci of schistosomiasis vectors at Forte beach, Itamaracá, Pernambuco, Brazil (KC Araújo, unpublished observations). 


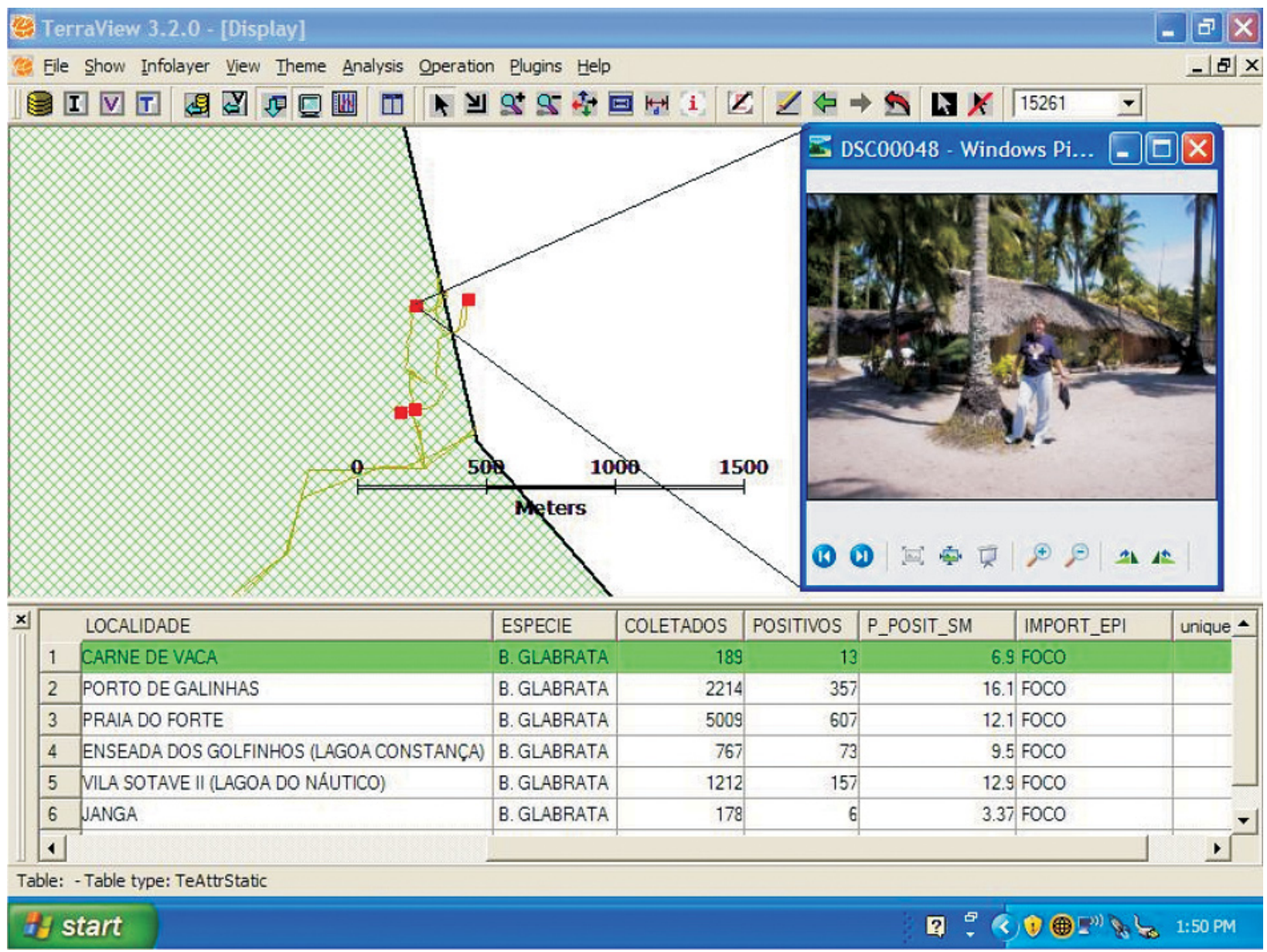

Fig. 6: application for monitoring the foci of schistosomiasis vectors at Carne de Vaca, Goiana, Pernambuco, Brazil (KC Araújo, unpublished observations). 


\begin{abstract}
$>$ SmBr18 (DQ137590.1)
ACTTACATGCATTACACACACTAGAACATCACAACGCACACAACCTGAACAACGGAAACATAACAGGGAACACCACTCCTCCCCA TAACCATCATTCACCAAACATTCAAACACCACTATTACAACGAAAAACAATCAACACATAATCAACCCAACACACTATATCCCAC ATTCACACACACACACACACACAAACACACTCCTTCATCAACATGTAGACAGAAAATGGAACACGACTACGCAATCAACATCGTC GTCCAACGAGAAATCTGTCCAACCATCAATGTCAACTCTCATTACCACCCACACATATGTTAACAAACAACAAGTGGACTTGGTT GTAGATGTACTTACCATGCATCTA
\end{abstract}

NCBI :

DQ137590.1 Schistosoma mansoni clone 169AAT microsatellite sequence

DQ137504.1 Schistosoma mansoni clone 082AAT microsatellite sequence DQ137605.1 Schistosoma mansoni clone 016CA microsatellite sequence

DQ137539.1 Schistosoma mansoni clone 118AAT microsatellite sequence

DQ137489.1 Schistosoma mansoni clone 067AAT microsatellite sequence

DQ137526.1 Schistosoma mansoni clone 105AAT microsatellite sequence

DQ137525.1

DQ137461.1

Schistosoma mansoni clone 104AAT microsatellite sequence

DQ137520.1

DQ137585.1

DQ137567.1

DQ137537.1

DQ137466.1

Schistosoma mansoni clone 038AAT microsatellite sequence Schistosoma mansoni clone 099AAT microsatellite sequence Schistosoma mansoni clone 164AAT microsatellite sequence Schistosoma mansoni clone 146AAT microsatellite sequence Schistosoma mansoni clone 116AAT microsatellite sequence Schistosoma mansoni clone 044AAT microsatellite sequence

$\begin{array}{lllll}\underline{673} & 673 & 100 \% & 0.0 & 100 \% \\ \underline{538} & 538 & 99 \% & 5 \mathrm{e}-150 & 93 \% \\ \underline{534} & 534 & 99 \% & 7 \mathrm{e}-149 & 93 \% \\ \underline{529} & 529 & 94 \% & 3 \mathrm{e}-147 & 94 \% \\ \underline{514} & 514 & 90 \% & 9 \mathrm{e}-143 & 94 \% \\ \underline{501} & 501 & 86 \% & 7 \mathrm{e}-139 & 95 \% \\ \underline{496} & 496 & 91 \% & 3 \mathrm{e}-137 & 93 \% \\ \underline{490} & 490 & 86 \% & 2 \mathrm{e}-135 & 94 \% \\ \underline{481} & 481 & 99 \% & 9 \mathrm{e}-133 & 91 \% \\ \underline{466} & 466 & 92 \% & 3 \mathrm{e}-128 & 92 \% \\ \underline{457} & 457 & 94 \% & 2 \mathrm{e}-125 & 90 \% \\ \underline{449} & 449 & 87 \% & 3 \mathrm{e}-123 & 92 \% \\ \underline{366} & 366 & 66 \% & 3 \mathrm{e}-98 & 93 \%\end{array}$

\title{
Alignment: NCBI
}

DQ137504

DQ137520

DQ137590

DQ137605

DQ137537

DQ137526

DQ137585

DQ137489

DQ137461

DQ137466

DQ137525

DQ137539

CONTIG-O

DQ137504

DQ137520

DQ137590

DQ137605

DQ137537

DQ137526

DQ137585

DQ137489

DQ137461

DQ137466

DQ137525

DQ137539

CONTIG-O

DQ137504

DQ137520

DQ137590

DQ137605

DQ137537

DQ137526

DQ137585

DQ137489

DQ137461

15

$1 \ldots$

5

15

25

35

45

$.1 \ldots$

$.1 \ldots$

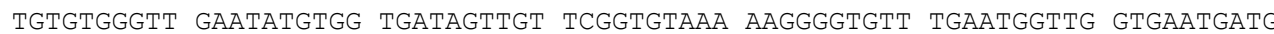

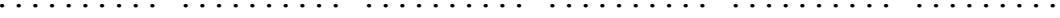

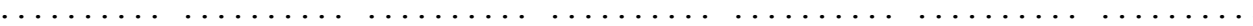

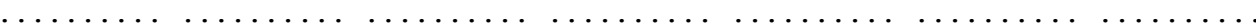

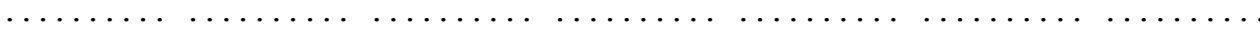

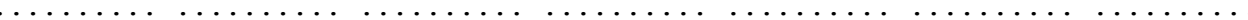

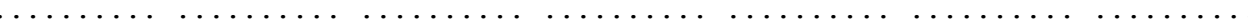

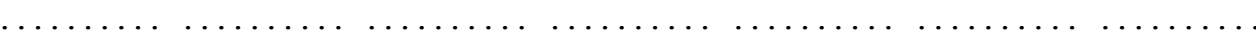

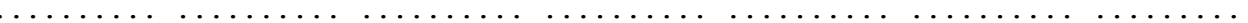

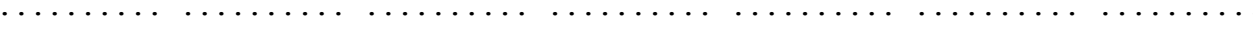

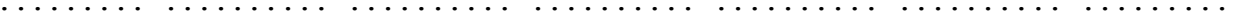

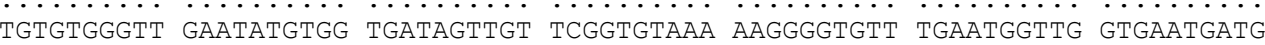

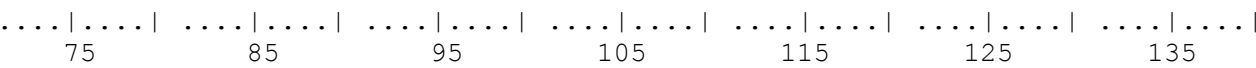

GGTTATGAgG AGGAGTGGTG TTCCCTGTTA TGTCTCCGTT GTTCAG-GTT GCGT-GTGTT GTGTG-TGTT

$\ldots \ldots \ldots \ldots \ldots \ldots \ldots \ldots \ldots \ldots \ldots \ldots \ldots$. $\ldots \ldots \ldots$ GTGTCGTGTT GTGTGGTGTT

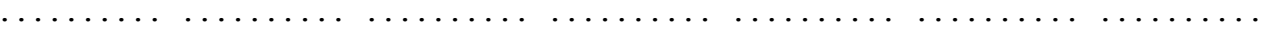

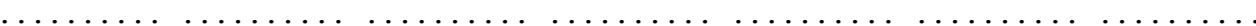

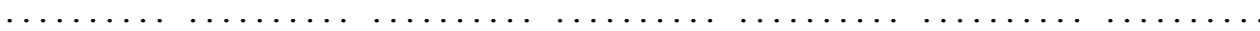

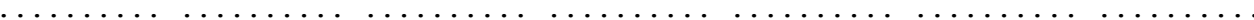

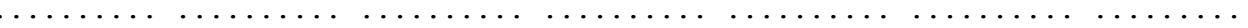

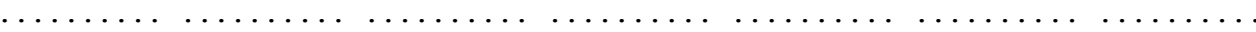

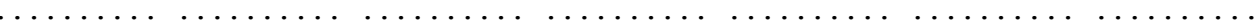

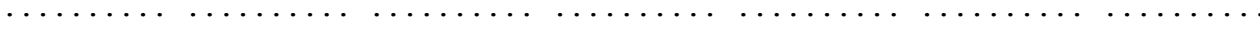

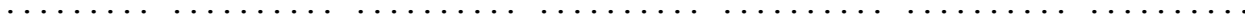

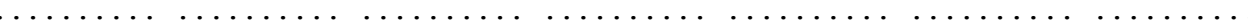

GGTTATGAGG AGGAGTGGTG TTCCCTGTTA TGTCTCCGTT GTTCAGTGTT GCGTCGTGTT GTGTGGTGTT

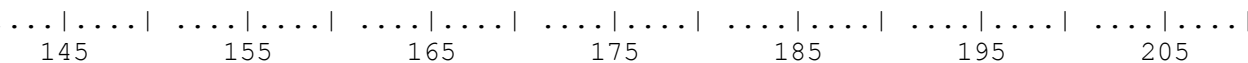

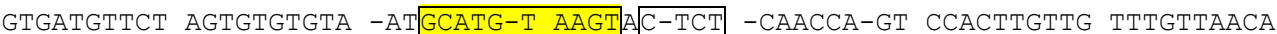
GTGATGTTCT AGTGTGTGTA -ATGCATG-T AA-TA C-TCT -CAACCA-GT CCACTTGTTG TTTGTTAACA

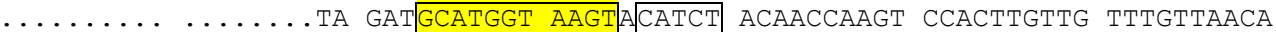

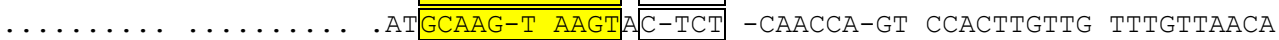
$\ldots \ldots \ldots \ldots \ldots \ldots \ldots \ldots \ldots$. . . . . . . . $\ldots \ldots \ldots \ldots \ldots \ldots \ldots \ldots \ldots$ C $\ldots \ldots \ldots \ldots \ldots \ldots \ldots \ldots \ldots \ldots \ldots \ldots \ldots \ldots \ldots$. $\ldots \ldots \ldots$. $\ldots \ldots$ CCACTTGTTG TTTGTTAACA $\ldots \ldots \ldots \ldots \ldots \ldots \ldots \ldots \ldots \ldots \ldots \ldots \ldots \ldots \ldots$ CCACTTGTTG TTTGTTAACA $\ldots \ldots \ldots \ldots \ldots \ldots \ldots \ldots \ldots \ldots \ldots \ldots \ldots \ldots \ldots \ldots \ldots \ldots \ldots \ldots$ 
$\mathrm{DQ} 137466$ DQ137525 DQ137539 CONTIG-0

DQ137504 DQ137520 DQ137590 DQ137605 DQ137537 DQ137526 DQ137585 DQ137489 DQ137461 DQ137466 DQ137525 DQ137539 CONTIG-0

DQ137504 DQ137520 DQ137590 DQ137605 DQ137537 DQ137526 DQ137585 DQ137489 DQ137461 DQ137466 DQ137525 DQ137539 CONTIG-O

DQ137504 DQ137520 DQ137590 DQ137605 DQ137537 DQ137526 DQ137585 DQ137489 DQ137461 DQ137466 DQ137525 DQ137539 CONTIG-0

DQ137504 DQ137520 DQ137590 DQ137605 DQ137537 DQ137526 DQ137585 DQ137489 DQ137461 DQ137466 DQ137525 DQ137539 CONTIG-0

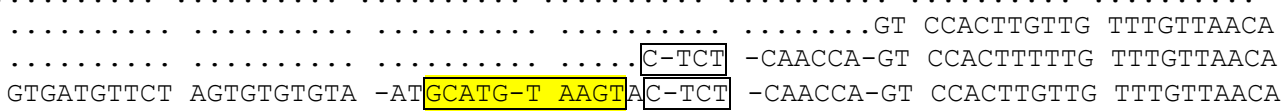
215
225
235
245
255
265
275 TATGT-GTGG GTG-TAATGA GAGT-GACAT TGATGGTTGG ACAGATTTCT CGTTGGACGA CGATGTTGAT TATGT-GTGG GTGGTAATGA GAGTTGACAT TGATGGTTGG ACAGATTTCT CGTTGGACGA CGATGTTGAT TATGT-GTGG GTG-TAATGA GAGT-GACAT TGATGGTGGG ACAGATTTCT CGTTGGACGA CGATGTTGAT TATGT-GTGG GTG-TAATGA GAGT-GACAT TGATGGTTGG ACAGATTTCT CGTTGGACGA CGATGTTGAT TATGT-GTGG GTG-TAATGA GAGT-GACAT TGAAGGTTGG ACAGATTTCT CGTTGGACGA AGATGTTGAT TATGT-GTGG GTG-TAATGA GAGT-GACAT TGATGGTTGG ACAGATTTCT CGTTGGACGA CGATGTTGAT TATGT-GTGG GTG-TAATGA GAGT-GACAT TGATGGTTGG ACAGATTTCT CGTTGGACGA CGATGTTGAT TATGTTGTGG GTG-TAATGA GAGT-GACAT TGATGGTTGG ACAGATTTCT CGTTGGACGA CGATGTTGAT

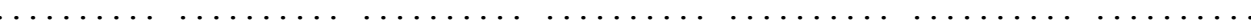
TATGT-GTGG TTG-TAATGT GAGT-GACAT TGTTGGTTGG ACAGATTTCT CGTTGGACGA CGATGTTGAT TATGT-GTGG GTG-TAATGA GAGT-GACAT TGATGGTTGG ACAGATTTCT CGTTGGACGA CGATGTTGAT TATGT-GTGG GTG-TAATGA GAGT-GACAT TGATGGTTGG ACAGATTTCT CGTTGGACGA CGATGTTGAT

$$
\begin{aligned}
& \ldots|\ldots| \ldots|\ldots| \ldots|\ldots| \ldots|\ldots| \ldots|\ldots| \ldots|\ldots| \ldots|\ldots| \ldots|\ldots| \ldots|\ldots| \\
& 285 \\
& 305
\end{aligned}
$$$$
295
$$

315

325

335

345 TTGCGTAG-T C-GTGTTCCA TTTTCTGTCT ACATG-TTGA TGAAGGAGTG TGTTTGTGTG TGTGTGTG-TTGCGTAG-T C-GTGTTCCA TTTTCTGTCT ACATG-TTGA TGAAGGAGTG TGT--GTGT- - T-TGTG-T-GCGTAG-T C-GTGTTCCA TTTTCTGTCT ACATG-TTGA TGAAGGAGTG TGTT $\overline{T G T G T G}$ TGTGTGTGTG TTGCGTAG-T C-GTGTTCCA TTTTCTGTCT ACATG-TTGA TGAAGGAGTG TGTTTGTGTG TGTGTGTGTG TTGCGTAG-T C-GTGTTCCA TTTTCTGTCT ACATGGTTGA TGAAGGAGTG TGTT TGTGTG TGTGTGTGTG TTGCGTAG-T C-GTGTTCCA TTTTCTGTCT ACATG-TTGA TGAAGGAGTG TGTT $\overline{\text { TGTGTG TGTGTGTGTG }}$ TTGCGTAG-T C-GTGTTCCA TTTTCTGTCT ACATG-TTGA TGAAGGAGTG TGT--GTGT- $--T-T G T G--$ TTGCGTAG-T C-GTGTTCCA TTT-CTGTCT ACATG-TTGA TGAAGGAGCG TGTT TGTGTG TGTGTGTGTG TTGCGTAG-T C-GTGTTCCA TTTTCTGTCT ACATG-TTGA TGAAGGAGTG TGTTTGTGTG TGTGTGTGTG ..GCGTAGAT CAGTGTTCCA ATTTCTGTCT ACATG-TTGA TGAAGGAGTG TGTTTGTGTG TGTGTGTGTG TTGCGTAG-T C-GTGTTCCA TTTTCTGTCT ACATG-TTGA TGAAGGAGTG TGTT TGTGTG TGTGTGT--G TTGCGTAG-T C-GTGTTCCA TTTTCTGTCT ACATG-TTGA TGAAGGAGTG TGTTTGTGTG TGTGTGTGTG TTGCGTAG-T C-GTGTTCCA TTTTCTGTCT ACATG-TTGA TGAAgGAgTG TGTT TGTGTG TGTGTGTGTG

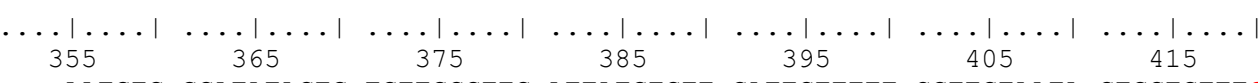
355
365
375
385
395
405
415

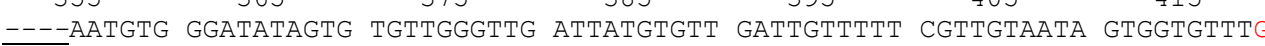
----AACGTG GGATATAGTG TGTTGGGTTG ATTATGTGTT GATTGTTTTT CGTTGTAATA GTGGTGTTTG TGTGAATGTG GGATATAGTG TGTTGGGTTG ATTATGTGTT GATTGTTTTT CGTTGTAATA GTGGTGTTTG ---AATGTG GGATATAGTG TGTTGGGTTG ATTATGTGTT GATTGTTTTT CGTTGTAATA GTGGTGTTTG TG--AATGTG GGATATAGTG TGTTGGGTTG GTTATGTGTT GATTGTTTC- CGTTGTAATA TTGGTGTTGG TG--AATGTG GGATATAGTG TGTTGGGTTG ATTATGTGTC GATTGTTTTT CGTTGTAATA GTGGCATTTG ----AACGTG GGATATAgTG TGTTGGGTTG ATTATGTGTT GATTGTTTTT CGTTGTAATA GTGGTGTTTG TG--AACGTG GGATATAGTG TGTTGGGTTG ATTATGTGTT GATTGTTTTT CGTTGTAATA GTGGTGTTTG

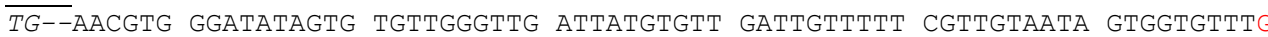
TG--AATGTG G-ATATAGTG TGTTGGGTTG ATTATGTGTT GATTGTTTTT CGTTGTAATA GTGGTGTTTG

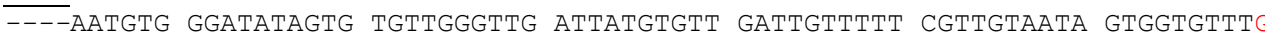
----AATGTG GGATATAGTG TGTTGGgTTG ATTATGTGTT GATTGTTTTT CGTTGTAATA GTGGTGTTTG

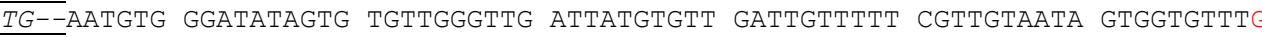

$$
\ldots|\ldots| \ldots|\ldots| \ldots|\ldots| \ldots|\ldots| \ldots|\ldots| \ldots|\ldots| \ldots|\ldots| \ldots|\ldots| \ldots \mid
$$

425

435

445

455

465

475

485 GT-TCAGGTT AATGTTTGGT GAATGATGGT TA-TGGGGAg GAGTG-GTGT TCCCTGTTAT GTTTCC-GTT GT-TCAGGTT AATGTTTGGT GAATGATGGT TA-TGGGGAG GAGTG-GTGT TCCCTGTTAT GTTTCC-GTT GT-TCAGGTT AATGTTTGGT GAATGATGGT TAATGgGGA AATTGTgTGT CCCCTGTAA- GTTTCCCGT- GTGTCAGGTT AATGTTTGGT GAATGATGGT TA-TGGGGAG GAGTG-GTGT TCC-TGTTAT GTTTCC-GTT GT-TCAGGTT AATGTTTGgT GAATGATGGT TA-TGGGGAg GAGTG-GTGT TCCCTGTTAT GTTTCC-GTT GT-TCAGGTT AATGTTTGGT GAATGATGGT TA-TGGGGAG GAGTG-GTGT TCCCTGTTAT GTTTCC-GTT GT-TCAGGTT AATGTTTGGT GAATGATGGT TA-TGGGGA GAGTG-GTGT TCCCTGTTAT GTTTCC-GTT GT-TCAGGTT AATGTTTGGT GAATGATGGT TA-TGGGGAG GAGTG-GTGT TCCCTGTTAT GTTTCC-GTT GT-TCAGGTT AATGTTTGGT GAATGATGGT TA-TGGGGAg GAGTG-GTGT TCCCTGTTAT GTTTCC-GTT GT-TCAG $\overline{\overline{G T T}}$ AATGTTTGGT GAATGATGgT TA-TGGGGAg GAGTG-GTGT TCCCTGTTAT GTTTCC-GTT GT-TCAGGTT AATGTtTGGT GAATGATGG TA-TGGGGAg GAGTG-GTGT TCCCTGTTAT GTTTCC-GTT GT-TCAGGTT

$$
\ldots|\ldots| \ldots|\ldots| \ldots|\ldots| \ldots|\ldots| \ldots|\ldots| \ldots|\ldots| \ldots|\ldots| \ldots|\ldots| \ldots|\ldots| \ldots|\ldots|
$$
495
505
515
525
535
545
555

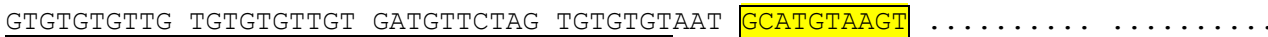

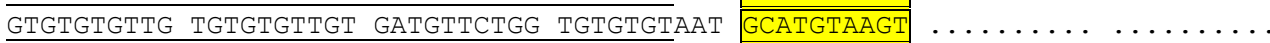
GTGT---- --- GCGTTGT GATGTTCTAG TGTGTGTAAT GCATGTAAGT $\ldots \ldots \ldots \ldots \ldots$ GTGTGTGTTG TGTGTGTTGT GATGTTCtAg TGTGTATAAT GCATGTAAGT $\ldots \ldots \ldots \ldots \ldots$ 
DQ137537 DQ137526 DQ137585 DQ137489 DQ137461 DQ137466 DQ137525 DQ137539 CONTIG-0

DQ137504 DQ137520 DQ137590 DQ137605 DQ137537 DQ137526 DQ137585 DQ137489 DQ137461 DQ137466 DQ137525 DQ137539 CONTIG-0

DQ137504 DQ137590 DQ137605 DQ137537 DQ137526 DQ137585 DQ137489 DQ137461 DQ137466 DQ137525

DQ137539 CONTIG-0 DQ137520

GTGTGTGTTG TGTGTGTTG.

GTGTGTGTTG TGTGTGTTGT GATGTTCTAG TG......

GTGTGTGTTG TGTGTGTTGT GATGTTCTAG TGTGTGTAAT GCATGT....

GTGTGTGTTG TGTGTGTTGT GATGTTCTAG TGTGTGTAAT GCATGTAAAT

GTGTGTGTTG TGTGTGTTGT GATGTTATAG TGTGTGTAAT

GTGTGTGTTG TGTGTGTTGT GATGTTCTAG TGTGTGTAAT GCATGTAAGT

GTGTGTGTTG TGTGTGTTGT GATGTTCTAG TGTGTGTAAT GCATGTAAGC

GTGTGTGTTG TGTGTGTTGT GATGTTCTAG TGTGTGTAAT GCATGTAAGT

GTGTGTGTTG TGTGTGTTGT GATGTTCTAG TGTGTGTAAT

GCATGTAAGT A CTCTCAACC AGTCCACTTG

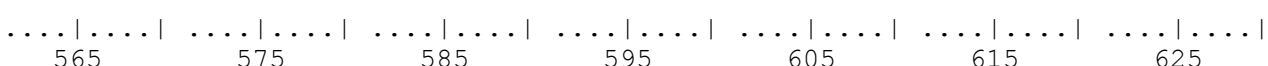

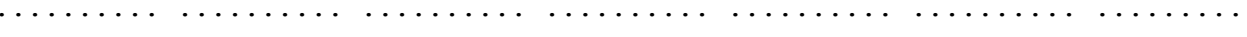

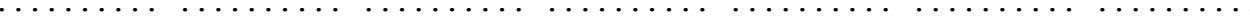

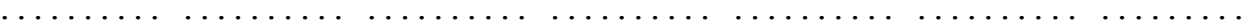

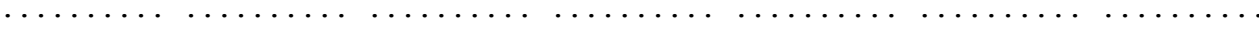

$\ldots \ldots \ldots+\cdots \cdots \cdots \cdots$

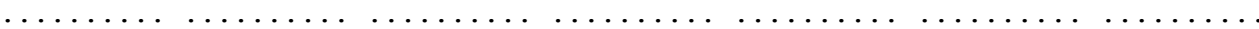

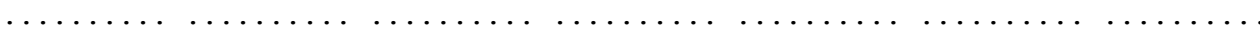

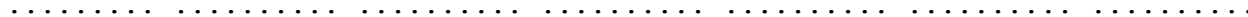

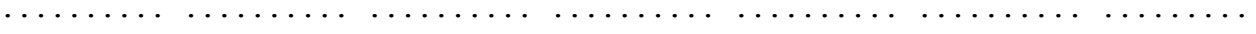

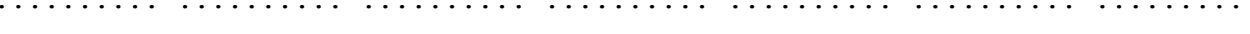

TTGTTTGCTA ACATATGTGT GGGTGTAATG AGAGTGTTCC CTGTTATGTT TCCGTTGTTC AGGTTGTGTG

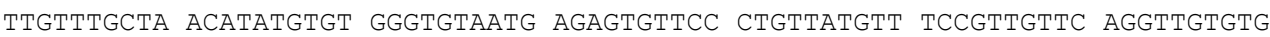

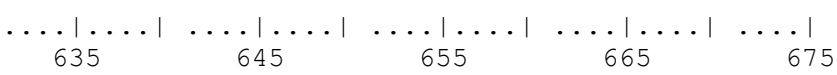

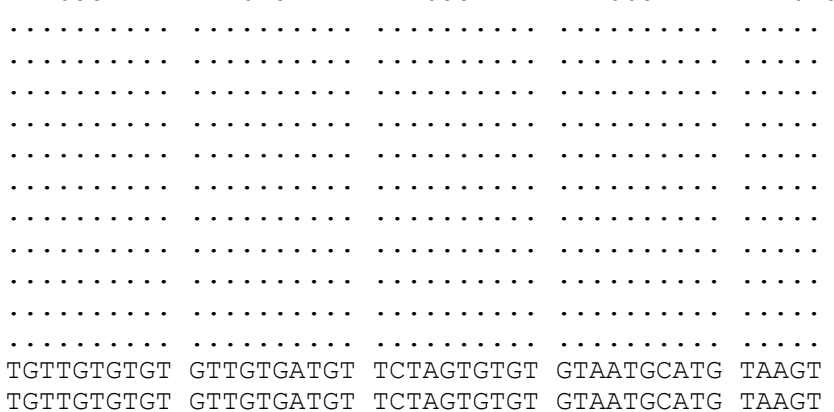

geneDB:

shisto $4743 \mathrm{c} 05 . \mathrm{p} 1 \mathrm{k}$

$14827.9 e-61 \quad 1$

S. mansoni predicted proteins [wublastx], for query: SmBr18 Sm02551

$2492.1 e-21 \quad 1$

S. mansoni predicted genes (coding sequences) [wublastn], for query: SmBr18 $\underline{\mathrm{Sm} 02551}$

$15595.3 e-66$

TIGR :

s mansoni|TC34704 homologue to UP|EGG3_SCHMA (P13396) Egg... 1491 2.0e-63 1
$4743 c 05$. Sm02551 TC34704 Contig-0

\section{Alignment: Genedb-TIGR}

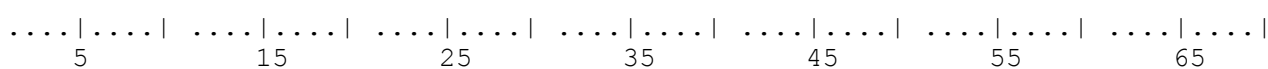

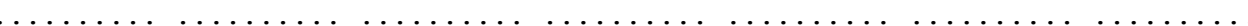

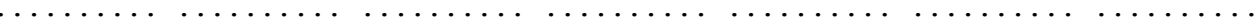
ACTATTACAG TGAAGAACAA TCAACACATA ATCAACCCAA CACACAATAT CCCACATTCA CACACACACA ACTATTACAG TGAAGAACAA TCAACACATA ATCAACCCAA CACACAATAT CCCACATT $\overline{\text { CA CACACACACA }}$

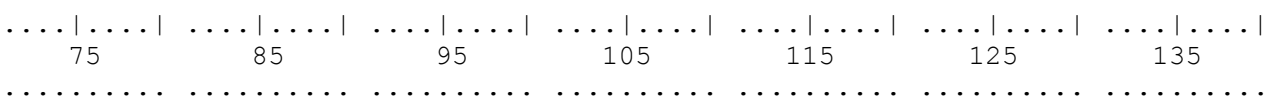
$\ldots \ldots \ldots \ldots \ldots \ldots \ldots \ldots \ldots \ldots \ldots \ldots \ldots$. $\ldots \ldots \ldots C-A \ldots A C A-$ CACACAACCT CACACAAACA CACTCCTTCA TCAACATGTA GACAGAAAAT GGAACACGAC TACGCAA-AT CA-ACATCGT CACACAAACA CACTCCTTCA TCAACATGTA GACAGAAAAT GGAACACGAC TACGCAACAT CACACAACCT 
$4743 c 05$. Sm02551 TC34704 Contig-0

$4743 c 05$. Sm02551 TC34704 Contig-0

$4743 c 05$. Sm02551 TC34704 Contig-0

$4743 c 05$. Sm02551 TC34704 Contig-0

$4743 c 05$. Sm02551 TC34704 Contig-0

$4743 \mathrm{c} 05$. Sm02551 TC34704 Contig-0

$4743 c 05$. Sm02551 TC34704 Contig-0

$4743 \mathrm{c} 05$. Sm02551 TC3470 4 Contig-0

$4743 \mathrm{c} 05$. Sm02551 TC34704 Contig-0

$4743 \mathrm{c} 05$. Sm02551 TC34704 Contig-0

$$
\ldots|\ldots| \ldots|\ldots| \ldots|\ldots| \ldots|\ldots| \ldots|\ldots| \ldots|\ldots| \ldots|\ldots| \ldots|\ldots| \ldots \mid
$$
145
155
165
175
185
195
205

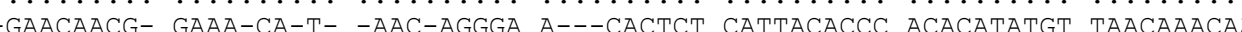
- GAAA-CA-T- -AAC-AGGGA A---CACTCT CATTACACCC ACACATATGT TAACAAACA CGTCCAACGA GAAATCTGTC CAACCATC-A ATGTCACTCT CATTACACCC ACACATATGT TAACAAACAA CGAACAACGA GAAATCAGTC CAACCAGCGA ATGTCACTCT CATTACACCC ACACATATGT TAACAAACAA

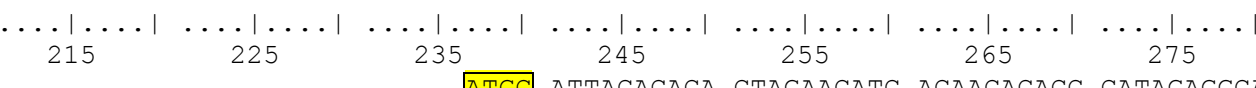

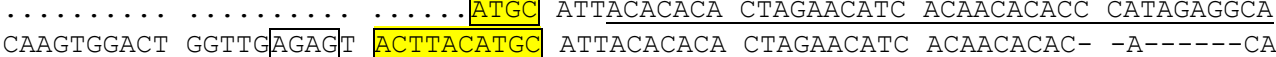
CAAGTGGACT GGTTGAGATT ACTTACATGC ATTACACACA CTAGAACATC ACAACACAC- -A-----CA CAAGTGGACT GGTTG AGAGT ACTTACATGC ATTACACACA CTAGAACATC ACAACACAC- -A------CA

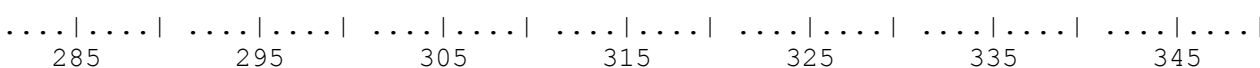
285
295
305
315

AC-C---- - ---GAACAA CGGAAACA-A ACAGGGAACA CC-CTACACG CCCATAACCA TCATTCACCA ACACACACAA GCCTGAACAA CGGAAACATA ACAGGGAACA CCACTCCTC- CCCATAACCA TCATTCACCA AC-C----- ---TGAACAA CGGAAACATA ACAGGGAACA CCACTCCTC- CCCATAACCA TCATTCACCA AC-C----- -- TGAACAA CGGAAACATA ACAGGGAACA CCACTCCTC- CCCATAACCA TCATTCACCA

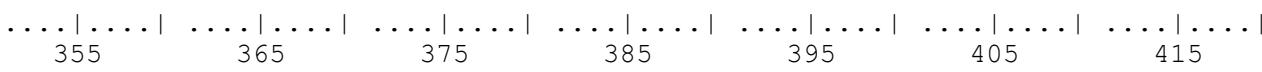

AACATTCAAA CACCACGATT ACAACGAAAA ACAATCAACA CATAATCAAC CCAACACACT ATATCCCACA AACATTCAAA CACCACTATT ACAACGAAAA ACAATCAACA CATAATCAAC CCAACACACT ATATCCCACA AACATTCAAA CACCACTATT ACAACGAAAA ACAATCAACA CATAATCAAC CCAACACACT ATATCCCACA AACATTCAAA CACCACTATT ACAACGAAAA ACAATCAACA CATAATCAAC CCAACACACT ATATCCCACA

$$
\ldots|\ldots| \ldots|\ldots| \ldots|\ldots| \ldots|\ldots| \ldots|\ldots| \ldots|\ldots| \ldots|\ldots| \ldots|\ldots| \ldots \mid
$$

$$
\begin{array}{lllllll}
425 & 435 & 445 & 455 & 465 & 475 & 485
\end{array}
$$

TTCACACACA CACACACACA AACACACTCC TTCATCAACA TGTAGACAGA AAATGGAACA CGACTACGCA TTCACACACA CACACACA-A A-CACACTCC TTCATCAACA TGTAGACAGA AAATGGAACA CGACTACGCA TTCACACACA CACACACACA AACACACTCC TTCATCAACA TGTAGACAGA AAATGGAACA CGACTACGCA TT CACACACA CACACACACA AACACACTCC TTCATCAACA TGTAGACAGA AAATGGAACA CGACTACGCA

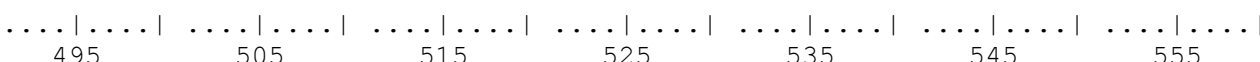
AATCAACATC GTCGTCCAAC GAGAAATCTG TCCAACCATC AATGTCACTC TCATTACACC CATACATATG AATCAACATC GTCGTCCAAC GAGAAATCTG TCCAACCATC AATGTCACTC TCATTACACC CACACATATG AATCAACATC GTCGTCCAAC GAGAAATCTG TCCAACCATC AATGTCACTC TCATTACACC CACACATATG AATCAACATC GTCGTCCAAC GAGAAATCTG TCCAACCATC AATGTCACTC TCATTACACC CACACATATG

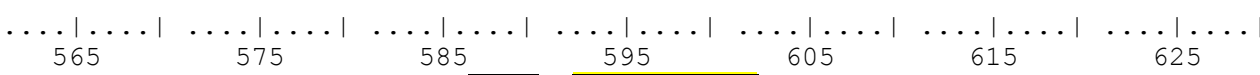

TTAACAAACA ACAAGTGGAC TGGTTG AGAG TACTTACATG CATTACACAC ACTAGAACAT CACAA----TTAACAAACA ACAAGTGGAC TGGTTG AGAG TACTTACATG CATTACACAC ACTAGAACAT CACAACACAC TTAACAAACA ACAAGTGGAC TGGTTGAGAG TACTTACATG CATTACACAC ACTAGAACAT CACAACACAC TTAACAAACA ACAAGTGGAC TGGTTGAGA TACTTACATG CATTACACAC ACTAGAACAT CACAACACAC

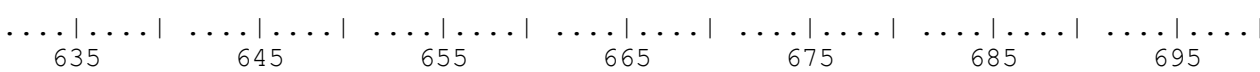
-------- ---CACACAC AACCTGAACA ACGGAAACAT AACAGGGAAC ACCACTCCTC CCCATAACCA ACAACACACA CAAC-C---- --- TGAACA ACGGAAACAT AACAGGGAAC ACCACTCCTC CCCATAACCA ACAACACACA CAACACACAC AACCTGAACA ACGGAAACAT AACAGGGAAC ACCACTCCTC CCCATAACCA ACAACACACA CAACACACAC AACCTGAACA ACGGAAACAT AACAGGGAAC ACCACTCCTC CCCATAACCA $\ldots|\ldots| \ldots|\ldots| \ldots|\ldots| \ldots|\ldots| \ldots|\ldots| \ldots|\ldots| \ldots|\ldots| \ldots|\ldots| \ldots|\ldots|$
705
715
725
735
745
755
765

TCATTCACCA AACATTCAAA CACCACGATT ACAACGAAAA ACAATCAACA CATAATCAAC CCAACACACT TCATTCACCA AACATTCAAA CACCACTATT ACAACGAAAA ACAATCAACA CATAATCAAC CCAACACACT TCATTCACCA AACATTCAAA CACCACTATT ACAACGAAAA ACAATCAACA CATAATCAAC CCAACACACT TCATTCACCA AACATTCAAA CACCACTATT ACAACGAAAA ACAATCAACA CATAATCAAC CCAACACACT

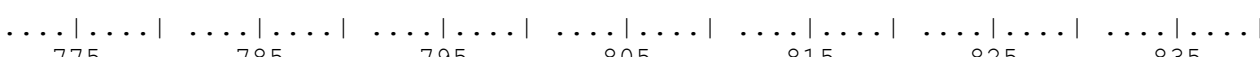
775
785
795
805
815
825
835

ATATCCCACA TTCACACACA CACACACACA AACACACTCC TTCATCAACA TGTAGACAGA AAATGGAACA ATATCCCACA TT CACACACA CACACACACA AACACACTCC TTCATCAACA TGTAGACAGA AAATGGAACA ATATCCCACA TTCACACACA CACACACA-A A-CACACTCC TTCATCAACA TGTAGACAGA AAATGGAACA ATATCCCACA TT CACACACA CACACACACA AACACACTCC TTCATCAACA TGTAGACAGA AAATGGAACA

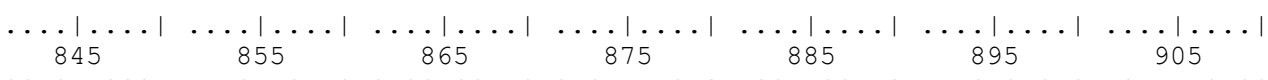
CGACTACGCA AATCAACATC GTCGTCCAAC GAGAAATCTG TCCAACCATC AATGTCACTC TCATTACACC CGACTACGCA AATCAACATC GTCGTCCAAC GAGAAATCTG TCCAACCATC AATGTCACTC TCATTACACC 
TC34704

Contig-0

$4743 c 05$. Sm02551 TC34704 Contig-0

$4743 c 05$. Sm02551 TC34704 Contig-0

$4743 c 05$. Sm02551 TC34704 Contig-0

$4743 c 05$. Sm02551 TC34704 Contig-0

$4743 c 05$. Sm02551 TC34704 Contig-0

$4743 c 05$. Sm02551 TC34704 Contig-0

$4743 c 05$. Sm02551 TC34704 Contig-0

$4743 c 05$ Sm02551 TC34704 Contig-0

$4743 c 05$. $\mathrm{Sm} 02551$ TC34704 Contig-0

$4743 c 05$. Sm02551 TC34704 Contig-0

CGACTACGCA AATCAACATC GTCGTCCAAC GAGAAATCTG TCCAACCATC AATGTCACTC TCATTACACC CGACTACGCA AATCAACATC GTCGTCCAAC GAGAAATCTG TCCAACCATC AATGTCACTC TCATTACACC

$$
\ldots|\ldots| \ldots|\ldots| \ldots|\ldots| \ldots|\ldots| \ldots|\ldots| \ldots|\ldots| \ldots|\ldots| \ldots|\ldots| \ldots|\ldots| \ldots|\ldots|
$$
915
925
935
945
955
965
975

CACACATATg tTAACAAACA ACAAgtgGAC TGgtTgAgA TACTTACATG CATTACACAC ACtAgAACAT CACACATATg tTAACAAACA ACAAGTGgAC TGgtTgAgA t ACTTACATG CATTACACAC ACTAGAACAT CACACATATG TTAACAAACA ACAAGTGGAC TGGTTG AGAG TACTTACATG CATTACACAC ACTAGAACAT

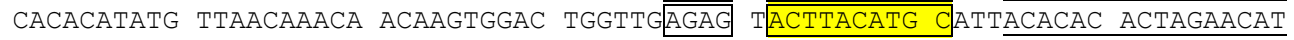

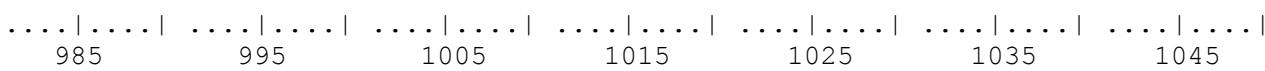

CACAACACAC ACAACACACA CAACCTGAAC AACGGAAACA TAACAGGGAA CACCACTCCT --C-CCCATA CACAACACAC ACAACACACA CAACCTGAAC AACGGAAACA TAACAGGGAA CACT-CTCAT TACACCCACA CACAACACAC ACAACACACA CAACCTGAAC AACGGAAACA TAACAGGGAA CACCACTCCT --C-CCCATA CACAACACAC ACAACACACA CAACCTGAAC AACGGAAACA TAACAGGGAA CACCACTCCT --C-CCCATA

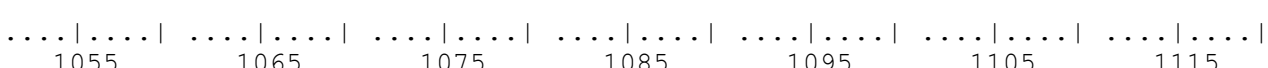
1055
1065
1075
1085
1095
1105
1115

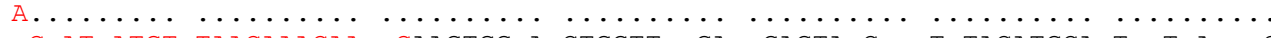
-C-AT-ATGT TAACAAACAA -CAAGTGG-A CTGGTT--GA -GAGTA-C-- T-TACATGCA T--T-A--C ACCATCAT-T CACCAAACAT TCAAACACCA CTA-TTACAA CGAAAAACAA TCAACA--CA TAATCAACCC ACCATCATGT CAACAAACAA TCAAACACCA CTAGTTACAA CGAAAAACAA TCAACATGCA TAATCAACCC

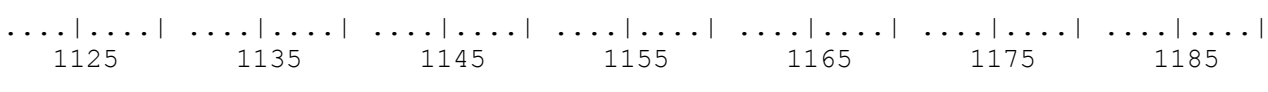
A-CACACTAG A----ACAT- CACA-ACACA CACA-ACAC- ---ACA-A-- CCTGAA-CAA CG-G-AAACA AACACACTAT ATCCCACATT CACACACACA CACACACACA CACACACACT CCTTCATCAA CATGTAGACA AACACACTAG ATCCCACATT CACACACACA CACACACACA CACACACACT CCTGAATCAA CATGTAAACA

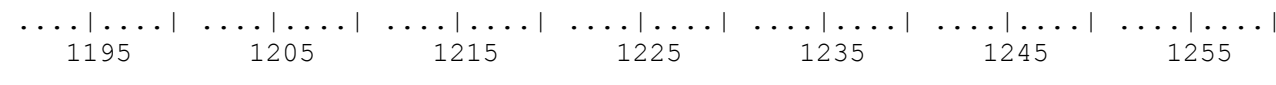
1195
1205
1225
1235
1245

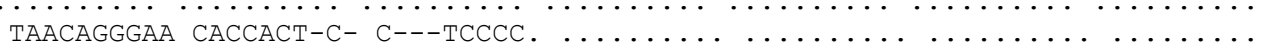

GAAAATGGAA CACGACTACG CAAATCAACA TCGTCGTCCA ACGAGAAATC TGTCCAACCA TCAATGTCAC GAAAAgGgAA CACCACTACG CAAATCAACA TCGTCGTCCA ACGAGAAATC TGTCCAACCA TCAATGTCAC

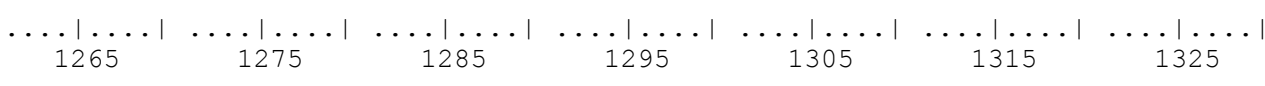

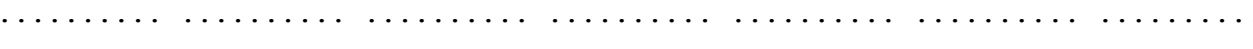

тCтCAтTACA CCCACACATA TGTTAACAAA CAACATGTG ACTGGTTGAG AGTACTTACA TGCATTACAC TCTCATTACA CCCACACATA TGtTAACAAA CAACATGtgG ACTGgtTgAg AGt ACTTACA TGCATTACAC

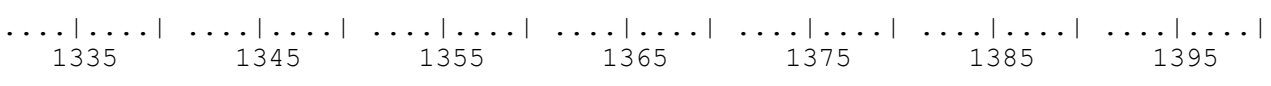

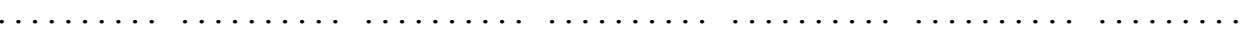

ACACTAGAAC ATCATCACAC CCAGTACAAC AACACCAACA ATTTGAAAAA CGAAACAGTC ACTCACACTC ACACTAGAAC ATCATCACAC CCAGTACAAC AACACCAACA ATTTGAAAAA CGAAACAGTC ACTCACACTC

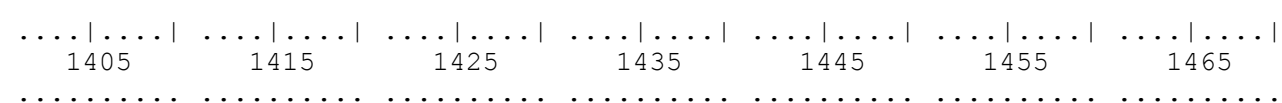

\begin{tabular}{|c|c|c|c|c|c|c|}
\hline$\cdots \mid{ }_{1475}{ }^{\prime}$ & $\cdots \mid{ }_{1485}{ }^{\prime}$ & $\cdots \mid{ }_{1495}$ & $\cdots\left|{ }_{1505} \cdots\right|$ & $\cdots \mid \begin{array}{l}1515 \\
15 \mid\end{array}$ & $\cdots \mid \begin{array}{l}\cdots \\
1525\end{array}$ & $\cdots\left|{ }_{1535} \cdots\right|$ \\
\hline & & 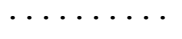 & $\cdots$ & $\cdots$ & $\cdot$ & \\
\hline
\end{tabular}

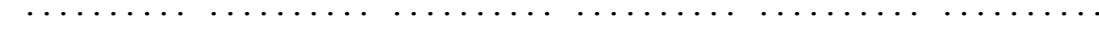

GTCTTCTTAG TAGCCATTGG TTACGCCACC GCCTACACCA CATCACATGA GTCTTCTTAG TAGCCATTGG TTACGCCACC GCCTACACCA CATCACATGA CTATTCGgGt GgGTACGGTG

\begin{tabular}{|c|c|c|c|c|c|}
\hline$\cdots+{ }_{1545} \cdots \mid$ & $\cdots||_{1555} \ldots \mid$ & $\cdots \underset{1565}{ }$ & $\cdots \mid \begin{array}{l}1575 \\
151\end{array}$ & $\cdots\left|{ }_{1585} \cdots\right|$ & $\begin{array}{c}\cdots|\ldots| \\
1595\end{array}$ \\
\hline
\end{tabular}

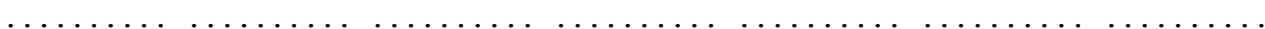

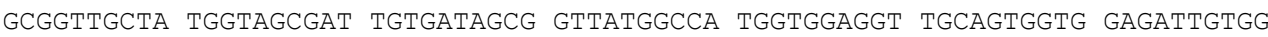

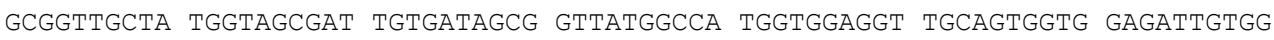

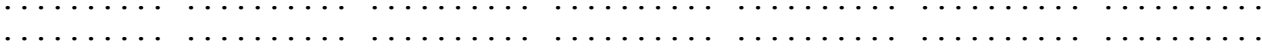

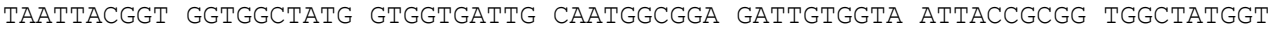

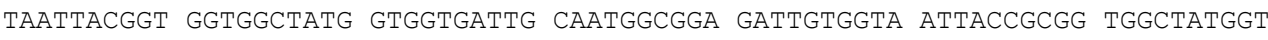

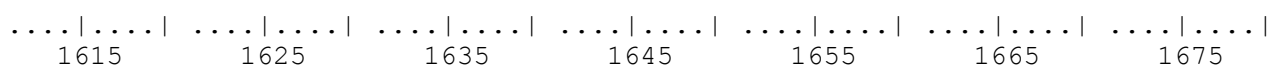


$4743 c 05$. $\mathrm{Sm} 02551$ TC34704 Contig-0

$4743 c 05$. Sm02551 TC34704 Contig-0

$4743 c 05$. Sm02551 TC34704 Contig-0

$4743 c 05$. Sm02551 TC34704 Contig-0

$4743 c 05$. Sm02551 TC34704 Contig-0

$4743 \mathrm{c} 05$. Sm02551 TC34704 Contig-0

NCBI GenedbTI Contig-0

NCBI GenedbTI Contig-0

NCBI

GenedbTI Contig-0

NCBI

GenedbTI Contig-0

NCBI GenedbTI Contig-0

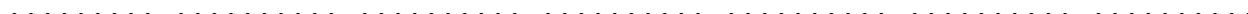

GGTGGGAATG GTGGACCCTG CTTTTTTGAC ACCCTCGCCC CGGCTTCGAT GAGGCCTTCC CTGCCCCCTA

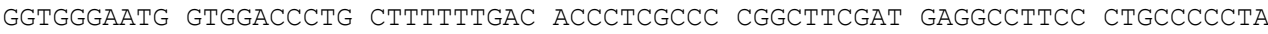

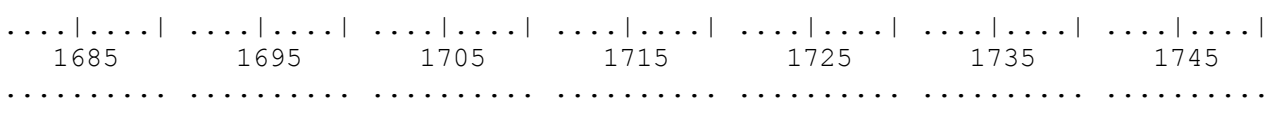

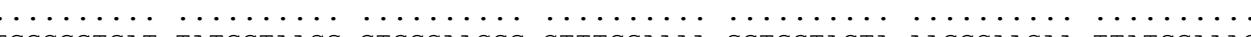
TGGCGGTGAT TATGGTAACG GTGGCAACGG CTTTGGAAAA GGTGGTAGTA AAGGCAACAA TTATGGAAAG

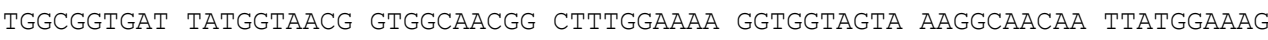

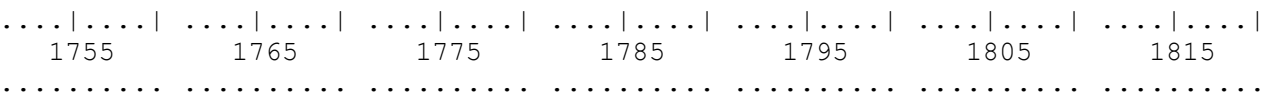
GgTTATGGCG GTGGTAGCGG TAAGGGTAA GGTGGTGGCA AAGGTGGCAA AgGCGGCAAA GGTGGCACTT

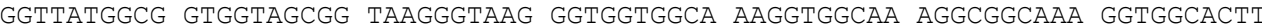

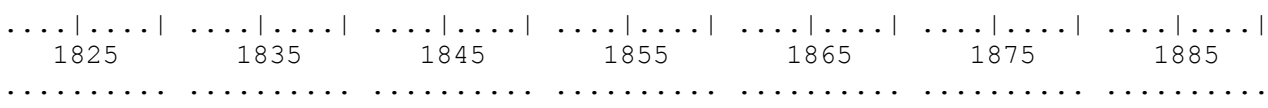

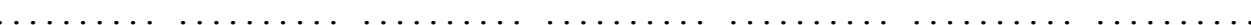
ACAAACCCAG CCATTATGGA GGCGGTTACT GAGGCACCAG TTGAGTTGTG GATCATTCTA ATTTGTTTGT ACAAACCCAG CCATTATGGA GgCGgTtACT GAgGCACCAG TTGAgTTGTG GATCATTCTA ATTTGTTTGT

\begin{tabular}{|c|c|c|c|c|c|c|}
\hline $\begin{array}{c}\ldots|\ldots| \\
1895\end{array}$ & $\begin{array}{c}\cdots|\ldots| \\
1905\end{array}$ & $\begin{array}{c}\ldots|\ldots| \\
1915\end{array}$ & $\begin{array}{c}\ldots|\ldots| \\
1925\end{array}$ & $\begin{array}{c}\ldots|\ldots| \\
1935\end{array}$ & $\begin{array}{c}\cdots|\ldots| \\
1945\end{array}$ & $\begin{array}{c}\ldots|\ldots| \\
1955\end{array}$ \\
\hline & & $\cdots$ & $\cdots \cdots$ & $\cdots \cdots$ & $\cdots \cdots$ & \\
\hline$\ldots$ & CA & $\ddot{C T A C}$ & $\ddot{\cdots T A}$ & & $\ddot{A T A}$ & \\
\hline
\end{tabular}
GTCACACTCT CCACTGTCCT ATTTTTCTAC ACACCTCTCA ATTCAACTCA CTGTAATATA GTCGTGTTTG

\section{Alignment: NCBI/Genedb/Tiger}

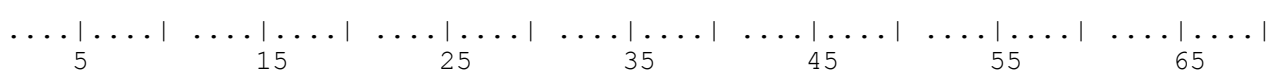
ACTATTACAG TGAAGAACAA TCAACACATA ATCAACCCAA CACACAATAT CCCACATTCA CACACACACA ACTATTACAg TGAAGAACAA TCAACACATA ATCAACCCAA CACACAATAT CCCACATTCA CACACACACA

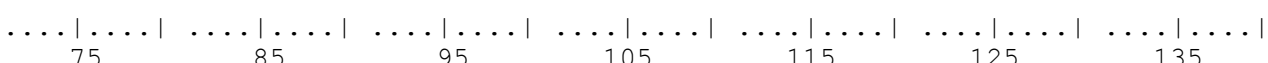
75
85
95
115
125
135

...... ACTTA CATGCATTAC AC--ACACTA GAACAT--CA CAAC-AC-AC ACAACA-CAC CACACAAACA CACTC-CTT- CAT-CA--AC ATGTAGAC-A GAAAATGGAA CA-CGACTAC GCAACATCAC CACACAAACA CACTCACTTA CATGCATTAC ACGTACACTA GAAAATGGAA CAACGACTAC ACAACATCAC

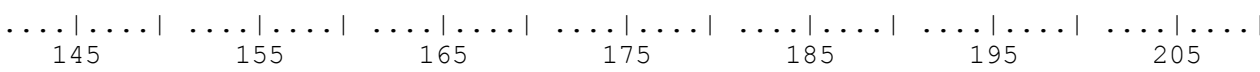

ACAACCT-GA ACAACG-GAA A-CA-T--AA C-AGGGAA-- -CACTCTCAT TACACCCACA CATATGTTAG ACAACCTCGA ACAACGAGAA ATCAGTCCAA CCAGCGAATG TCACTCTCAT TACACCCACA CATATGTTAA ACAACCTCGA ACAACGAGAA ATCAGTCCAA CCAGCGAATG TCACTCTCAT TACACCCACA CATATGTTAA

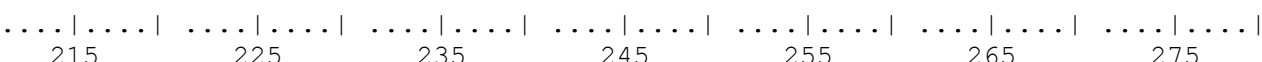

AAACAACAA GTGGACTGGT TGAGAGTACT TACATGCATT ACACACACTA GAACATCACA ACACACACAA CAAACAACAA GTGGACTGGT TGAGAGT ACT TACATGCATT ACACACACTA GAACATCACA ACACACACAA CAAACAACAA GTGGACTGGT TGAGAT ACT TACATGCATT ACACACACTA GAACATCACA ACACACACAA

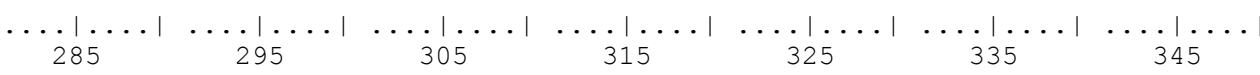

CACACACAAC CTGAACAACG GAAACATAAC AGGGAACACC ACTCCTCCCC ATAACCATCA TTCACCAAAC C-C------ -TGAACAACG GAAACATAAC AGGGAACACC ACTCCTCCCC ATAACCATCA TTCACCAAAC CACACACAAC CTGAACAACG GAAACATAAC AGGGAACACC ACTCCTCCCC ATAACCATCA TTCACCAAAC 
NCBI

GenedbTI

Contig-0

NCBI

GenedbTI

Contig-0

NCBI

GenedbTI

Contig-0

NCB I

GenedbTI

Contig-0

NCBI

GenedbTI

Contig-0

NCBI

GenedbTI

Contig-0

NCBI

GenedbTI

Contig-0

NCBI

GenedbTI

Contig-0

NCBI

GenedbTI

Contig-0

NCBI

GenedbTI Contig-0

NCBI

GenedbTI Contig-0

NCBI

GenedbTI

Contig-0

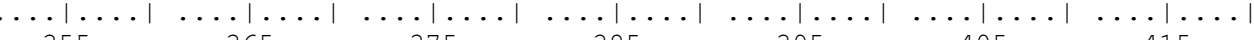
$405 \quad 415$ ATTCAAACAC CACTATTACA ACGAAAAACA ATCAACACAT AATCAACCCA ACACACTATA TCCCACATTC ATTCAAACAC CACTATTACA ACGAAAAACA ATCAACACAT AATCAACCCA ACACACTATA TCCCACATTC ATTCAAACAC CACTATTACA ACGAAAAACA ATCAACACAT AATCAACCCA ACACACTATA TCCCACATT $\underline{\underline{C}}$

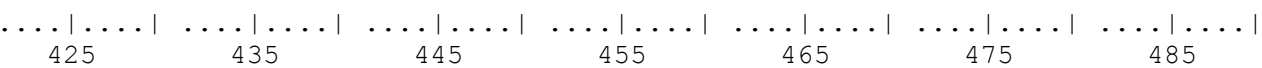
ACACACACAC ACACACAAAC ACACTCCTTC ATCAACATGT AGACAGAAAA TGGAACACGA CTACGCAAAT ACACACACAC ACACACAAAC ACACTCCTTC ATCAACATGT AGACAGAAAA TGGAACACGA CTACGCAAAT ACACACACAC ACACACAAAC ACACTCCTTC ATCAACATGT AGACAGAAAA TGGAACACGA CTACGCAAAT

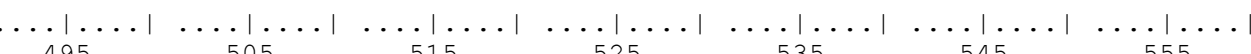
505
515
525
555

CAACATCGTC GTCCAACGAG AAATCTGTCC AACCATCAAT GTCACTCTCA TTACACCCAC ACATATGTTA CAACATCGTC GTCCAACGA AAATCTGTCC AACCATCAAT GTCACTCTCA TTACACCCAC ACATATGTTA CAACATCGTC GTCCAACGAG AAATCTGTCC AACCATCAAT GTCACTCTCA TTACACCCAC ACATATGTTA

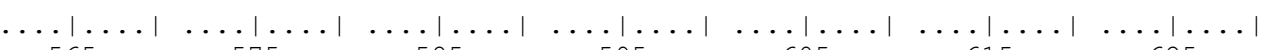
565
575
585
595
605
615
625

ACAAACAACA AgTGGACTGg tTGAGAGT AC TTACATGCAT TACACACACT AGAACATCAC AACAC-CACA ACAAACAACA AGTGGACTGG TTGAGAGT AC TTACATGCAT TACACACACT AGAACATCAC AACACACACA ACAAACAACA AGTGGACTGG TTGAGAT AC TTACATGCAT TACACACACT AGAACATCAC AACACACACA

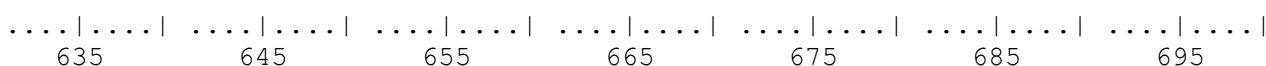
645
665
675
695

-CA-ACACGA CGCA-ACA-C -TGAACAACG GAGACATAAC AGGGAACACC ACTCCTCCTC ATAACCCATC ACACACACAA CACACACAAC CTGAACAACG GAAACATAAC AGGGAACACC ACTCCTCCCC ATAACC-ATC ACACACACAA CACACACAAC CTGAACAACG GAAACATAAC AGGGAACACC ACTCCTCCCC ATAACCCATC

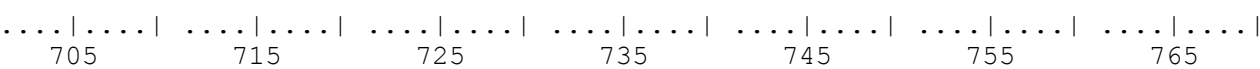
ATTCACCAAC CATTCAAACA CCCCTTTTTA CACCGAACAA CTATCACCAC ATATTCAACC CA-CACA... ATTCACCAAA CATTCAAACA CCACTATT-A CAACGAAAAA CAATCAACAC ATAATCAACC CAACACACTA ATTCACCAAA CATTCAAACA CCACTATTTA CAACGAAAAA CAATCAACAC ATAATCAACC CAACACACTA

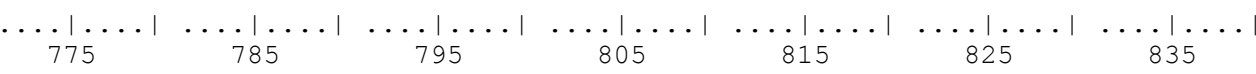

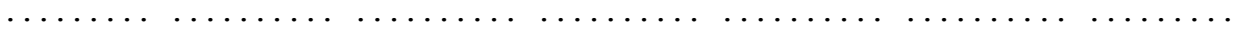
TATCCCACAT TCACACACAC ACACACACAA ACACACTCCT TCATCAACAT GTAGACAGAA AATGGAACAC TATCCCACAT TCACACACAC ACACACACAA ACACACTCCT TCATCAACAT GTAGACAGAA AATGGAACAC

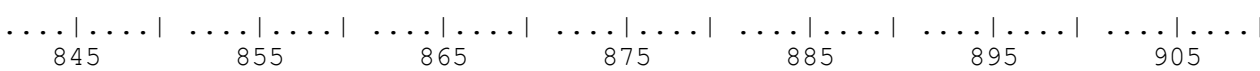

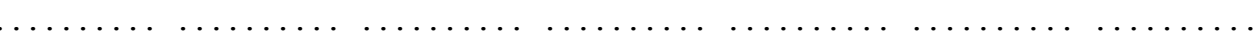
GACTACGCAA ATCAACATCG TCGTCCAACG AGAAATCTGT CCAACCATCA ATGTCACTCT CATTACACCC GACTACGCAA ATCAACATCG TCGTCCAACG AgAAATCTGT CCAACCATCA ATGTCACTCT CATtACACCC

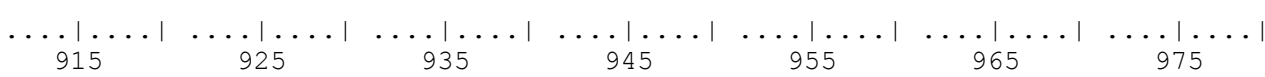

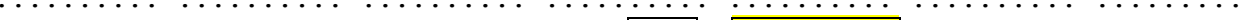
ACACATATGT TAACAAACAA CAAGTGGACT GgTTGAgAG ACTTACATGC ATTACACACA CTAGAACATC ACACATATGT TAACAAACAA CAAGTGGACT GGTTGAGAT ACTTACATGC ATTACACACA CTAGAACATC

\begin{tabular}{|c|c|c|c|c|c|}
\hline$\cdots\left|{ }_{985} \ldots\right|$ & $\cdots{ }_{995} \ldots$ & $\begin{array}{c}\cdots|\cdots| \\
1005\end{array}$ & $\begin{array}{c}\cdots|\ldots| \\
1015\end{array}$ & $\begin{array}{c}\cdots|\ldots| \\
1025\end{array}$ & $\begin{array}{c}\cdots|\ldots| \\
1035\end{array}$ \\
\hline
\end{tabular}

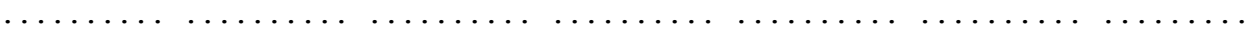
ACAACACACA CAACACACAC AACCTGAACA ACGGAAACAT AACAGGGAAC ACCACTCCTC CCCATAACCA ACAACACACA CAACACACAC AACCTGAACA ACGGAAACAT AACAGGGAAC ACCACTCCTC CCCATAACCA

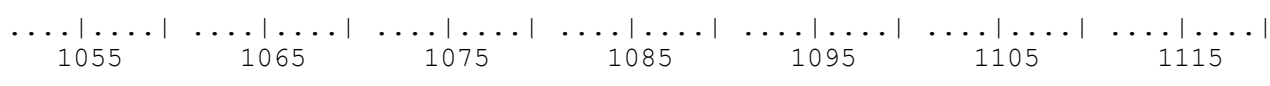

TCATGTCAAC AAACAATCAA ACACCACTAG TTACAACGAA AAACAATCAA CATGCATAAT CAACCCAACA TCATGTCAAC AAACAATCAA ACACCACTAG TTACAACGAA AAACAATCAA CATGCATAAT CAACCCAACA

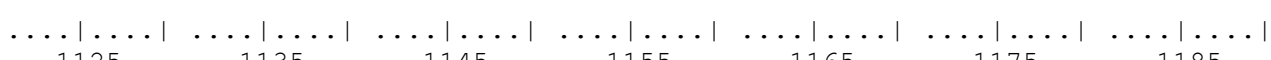
1125
1135
1145
1155
1165
1175
1185

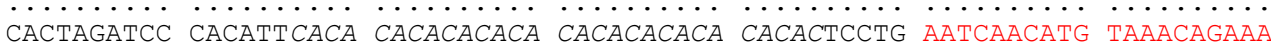
CACTAGATCC CACATTCACA CACACACACA CACACACACA CACACTCCTG AATCAACATG TAAACAGAAA

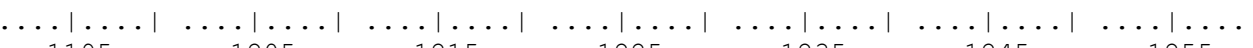

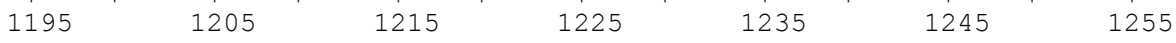


NCBI

GenedbTI Contig-0

NCBI

GenedbTI Contig-0

NCBI

GenedbTI

Contig-0

NCB I

GenedbTI

Contig-0

NCBI

GenedbTI Contig-0

NCBI

GenedbTI Contig-0

NCBI

GenedbTI

Contig-0

NCB I

GenedbTI

Contig-0

NCBI

GenedbTI Contig-0

NCBI

GenedbTI

Contig-0

NCBI

GenedbTI

Contig-0

NCBI

GenedbTI

Contig-0
AGGGAACACC ACTACGCAAA TCAACATCGT CGTCCAACGA GAAATCTGTC CAACCATCAA TGTCACTCTC AGGGAACACC ACTACGCAAA TCAACATCGT CGTCCAACGA GAAATCTGTC CAACCATCAA TGTCACTCTC

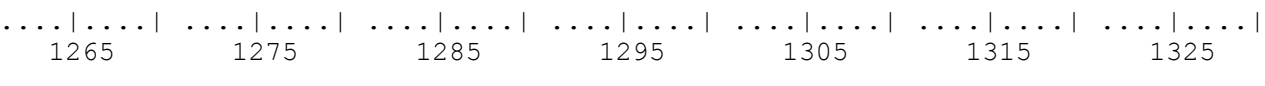
ATtACACCCA CACATATGTt AACAAACAAC ATgtggactg GTtg AgAG A CTTACATGCA TTACACACAC ATTACACCCA CACATATGTT AACAAACAAC ATgTgGACTG GTTGAGAGT A CTTACATGCA TTACACACAC

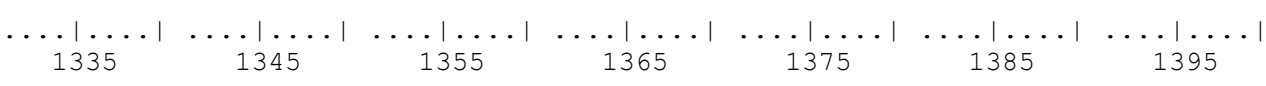

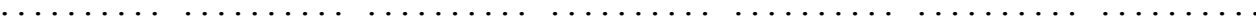
TAGAACATCA TCACACCCAG TACAACAACA CCAACAATTT GAAAAACGAA ACAGTCACTC ACACTCGTCT TAGAACATCA TCACACCCAG TACAACAACA CCAACAATTT GAAAAACGAA ACAGTCACTC ACACTCGTCT

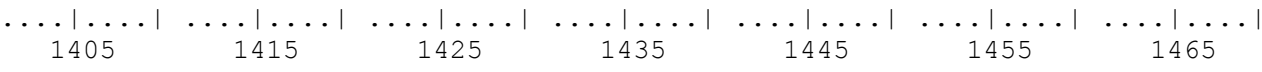

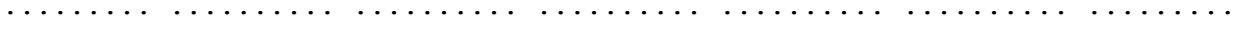
TCTTAGTAGC CATTGGTTAC GCCACCGCCT ACACCACATC ACATGACTAT TCGgGTGGGT ACGGTGGCGG TCTTAGTAGC CATTGGTTAC GCCACCGCCT ACACCACATC ACATGACTAT TCGgGTGGgt ACGGTGGCGG

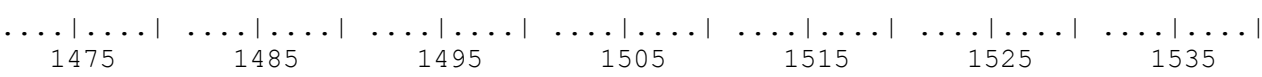
$\ldots \ldots \ldots \ldots \ldots \ldots \ldots \ldots \ldots \ldots \ldots \ldots$

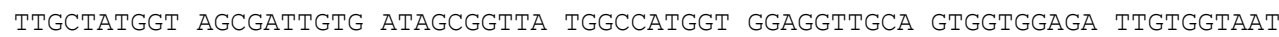

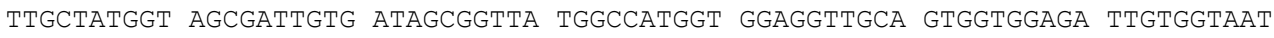

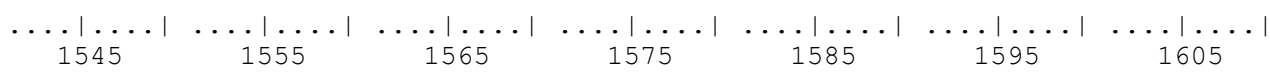

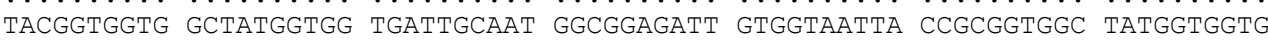

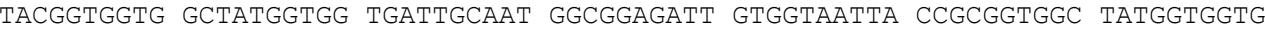

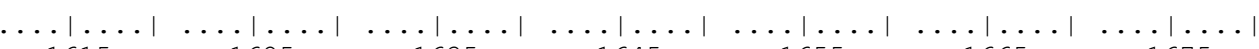
1615
1625
1635
1645
1655
1665
1675

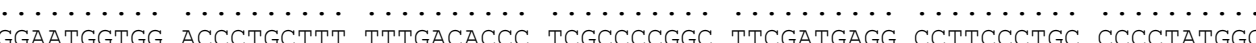

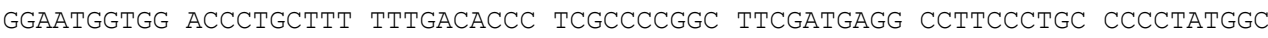

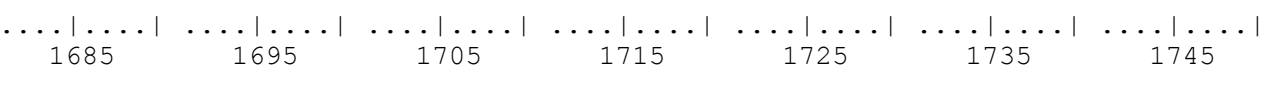

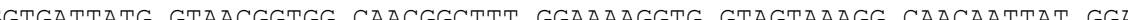

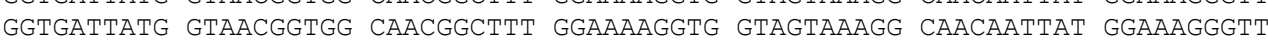

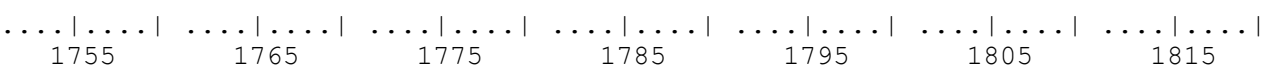

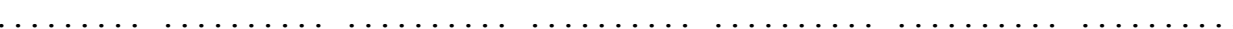

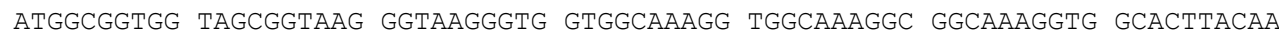

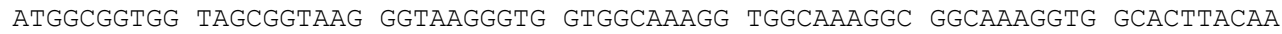 $\ldots \mid \ldots \ldots$
1825
1845
1855
1875

1835

1865

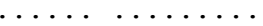
ACCCAGCCAT TATGGAGGCG GTTACTGAGg CACCAGTTGA GTTGTGGATC ATTCTAATTT GTTTGTGTCA

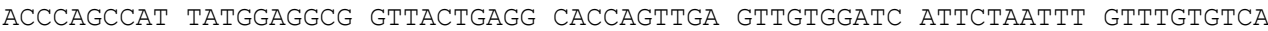

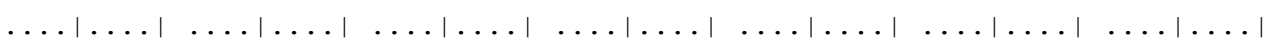

$\begin{array}{llllll}1895 & 1905 & 1915 & 1925 & 1935 & 1945\end{array}$

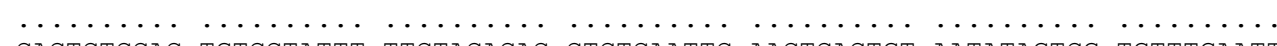
CACTCTCCAC TGTCCTATTT TTCTACACAC CTCTCAATTC AACTCACTGT AATATAGTCG TGTTTGAATT CACTCTCCAC TGTCCTATTT TTCTACACAC CTCTCAATTC AACTCACTGT AATATAGTCG TGTTTGAATT

$$
\cdots 1 \ldots 1 \cdots
$$

CGAGATGAAT

CGAGATGAAT AAA 
>Smp_scaff000011

TTCCĀGTATTTAAAATTCATCAGGGGAAATAATATATGTGGTCTTTTAATGCTACAGTTTGTATTGTATACTTAGGCTGACAATT TTCAACTTGTTAGAATTTTGCTTAAATTAGGAATAATTTGTTCTTGTTCTTGTGGTTGTGTGGTTTCATTCAGTTTCATATATA TCTATTCTTTATTGGACTGAGCTTCAACCTTGCTAGTCGAATCCCAGTTATTTGGTAATTTAATCTGGGTCGCATTTGTTTCTTG GTGTTTGTTAGTTGAAATTGGAAGCCACAAGTATTTGATTTATGTAATTCAGTATTATTTTGCAGTTTGGCATTGGTGCGAAATC AGTGCATTCTTCAACAAAACCCACCTGTCGTTTCACCTGTTTCCTCAAGTCAGCACAATCCTTTGATTTCATCTCCGAGCGTTCG TGGATTAATGTTACCAAATATCCAACCCAATGATTTATCTCTTTCCTCATACCTTTCACGTTTTCCTGTTCAACCAACAAATGCC GAGTCTAAGCAGTCTGTTAGTCTGTCATCAACTATTATACCGTGTTCAAAACAACCATCAGGATTCGTCGCTTCACTCGAGGAAG TGTCCACAGTTAAAGAAAGCGACCCAAACCGTGGTGGTTGGATGACAGATTATGAAGCAATTGGTGTGTTATTGATTCAGTTGCG TCCTTTAATGGTATCTAATCCCTATGTCCAGGTCAGTTTCTTATTTTCTGAATGAGTTTCTAATTTACTACACTTTTAGTTGATT ATGTGATTCTTAAGTAGCAGAGTTGCTTGAACATTAATGCCTCTGATCACTGAATGATTTTGTCGGCTGGATCGTTTATGTCTTT TCATTCACTGTAAGTGAAACATAGGAGCTGTAGTTCTTAGCACTTGATGAAAATTAATCAAGGTTAATAGTTCCGTGATAAGTCA CTTATTTACAGCAGTATGAAGCACCACATTATTAGGGCTATGCGGTAATAAAAATGTAGGTCAAACCTCTCCATATATCTGTTTA GTGGATATAATTTGGGCGAAATTGCCTGTTTTGAAACGTCTTTTAATACAGTTTGAAAGTTGATACACCATATATTTATCATCAA AATGATGGAATTCGACATCAAAATACTCGTTCTTTGAATTTCAGGTTCAATGAAATCCCACTTGTTTCGGATGATTTCCTTCAAG AATAATTTGCTATAACTTCTGAGGATTTTGGCACAGTTGATAGTTTTTTCAACGGTTAATTGTAATAAATAGTTGTCTATTTATC TCAAATTATGTATTCAAATTTCACATAACAAATAATCAAATTCTAACAAACAATTATTAGTTAGCCTATAATTTATTATGGGCAA ATATGTATGGTTGTACAGCTTTGAAAATGAATTATTTGCTGAGTTAATCATTTTATTATTCAAGAAGTGTTTTTTAGAATTAATT TCCGAGCTAATATAGATTGAATTTCATGTATGTGTCCTTAAGACCATGAATTTCCATGGCTTGTTTATTCATACTCAGATCAATG TCTCTTTTGATTTTGAACTTTCTATGTAAAAGTGACCAGGAACTTCCGAGGCTTATTCATTTCTATCTTGAATAACCGAAATGCA TCGGTGTCCATAACTGAGTAATACCCAGAATGTTGATTCTGTTACCAGTTGTTCCGTCATTTGACTTAAAATGCATTACTGCTTG ATATACTGTAATTCAGTTAATTTCTGTGTCATGAGTGGAAAATGTAGTGGTTGTTTTATTCAGACAAATTTTTAGTTGTTAGTTT AACGGTATAGTAGTTTCTCCATTTCGAGACATAATCTCACTGTTATAGTTGTTTTTAATCTCCCAGCTATACAACAAAATAAAAA TAAATAACATCCGAAAATTGGCTAATTTCTAAGTTTGAATTTTGTTTAGGTATACGTTTATTGCTGTCAATGTACCTGAGCGTCT TTCTTTTTGTCGTGAACAAGTCTAATAATGATATCGTACACTGACTAGTTATTCAAGGACTACTAACCATTATTTCTCTTGCCAG GATTATTATTTTGCATCTCAATGGCTGCGACGAATGAATGCTATTCAAGCTAAGCATATTTCAAAACATAAAATGAACACTACAA CTTCACCAGCAATAGTGCAGCTTCCTATTCCTATACCATTGGAAAGCTTGAGCGATTCCAATTCATCATATCAAGTAACTCTAAA ACATAAACTTGTTATTCCGTTTCGTGCTGTGAATCGCAATCCTGGCTCTGACCAAAATGTGGATGAAAGCTGTGGCAGCTTAGAA AAAAAAAGTGTATGTTCAGAAACTCCTACATCTGAATCAAGTAATGCACTGGGTCGTCCTACTAGATCTAATGTTCATCGACCCC GTGTAGTAGCAGAGTTATCTCTGGCGTCCGCGATTTCATCTACTTCAGAAAACTCTTATCCAATGATACACATCGATAACCATTC ACTGAATAGAAAAGTAAGTCACACAATATAATACAAAATAATCAATACAATAATAAACACGGAAAATGCGCATCAATTTCTCCCG GAGTGTGCGAAATGTTCAAGTCTTATCAGAACATGAATGAAGGATATTGTGTGTTGGGTTGATTATATGTTGATTGTTTTTCGTT GTAATAGTGGTGTTTGAATGTTTGGTGAATGATGGTTATGGGGAGGAGTGGTGTTCCCTGTTATGTTTCCGTTGTTCAGGTTGTG TGTGTTGTGTGTGTTGTGTGTGTTGTGATGTTCTAGTGTGTGTAATGCATGTAAGTACTCTCGACCAGTCCACTTGTTGTTTGTT AACATATGTGTGGGTGTAATGAGAGTGACATTGATGGTTGGACAGATTTCTCGTTGGACGACGATGTTGATTTGCGTAGTCGTGT TCCATTTTCTGTCTACATGTTGATGAAGGAGTGTGTTTGTGTGTGTGTGTGTGTGTGCATGTGGGATATAGTGTGTTGGGTTGAT TATGTGTTGATTGTTTTTCGTTGTAATAGTGGTGTTTGAATGTTTGGTGAATGATGGTTATGGGGAGGAGTGGTGTTCCCTGTTA TGTTTCCGTTGTTCAGGTTGTGTGTGTTGTGTGTGTTGTGATGTTCTAGTGTGTGTAATGCATGTAAGTACTCTCAACCAGTCCA CTTGTTGTTTGTTAACATATGTGTGGGTGTAATGAGAGTGACATTGATGGTTGGACAGATTTCTCGTTGGACGACGATGTTGATT TGCGTAGTCGTGTTCCATTTTCTGTCTACATGTTGATGAAGGAGTGTGTTTGTGTGTGTGTGTGTGTGTGAATGTGGGATATTGT GTGTTGGGTTGATTATGTGTTGATTGTTTTTCGTTGTAATAGTGGTGTTTGAATGTTTGGTGAATGATGGTTATGGGGAGGAGTG GTGTTCCCTGTTATGTTTCCGTTGTTCAGGTTGTGTGTGTTGTGTGTGTTGTGTGTGTTGTGATGTTCTAGTGTGTGTAATGCAT GTAAGTACTCTCAACCAGTCCAATTGTTGTTTGTTAACATATGTGTGGGTGTAATGAGAGTGACATTGATGGTTGGACAGATTTC TCGTTGGACGACGATGTTGATTTGCGTAGTCGTGTTCCATTTTCTGTCTACATGTTGATGAAGGAGTGTGTGTGTGTGTGTGTGA ATGTGGGATATAGTGTGTTGGGTTGATTATGTGTTGATTGTTTTTCGTTGTAATAGTGGTGTTTGAATGTTTGGTGAATGATGGT TATGGGGAGGAGTGGTGTTCCCTGTTATGTTTCCGTTGTTCAGGTTGTGTGTGTTGTGTGTGTTGTGATGTTCTAGTGTGTGTAA TGCATGTAAGTACTCTCAACCAGTCCACTTGTTGTTTGTTAACATATGTGTGGGTGTAATGAGAGTGACATTGATGGTGGACAGA

$>$ Smp scaff000068

TGTTGATTGTTTTTCGTTGTATAGTGGTGTTTGAATGTTTGGTGAATGATGGTTATGGGGAGGAGTGGTGTTCCCTGTTATGTTT CCGTTGTTCAGGTTGTGTGTGTTGTGTGTGTTGTGTGTGTTGTGATGTTCTAGTGTGTGTAATGCATGTAAGTACTCTCAACCAG TCCACTTGTTGTTTGTTAACATATGTGTGGGTGTAATGAGAGTGACATTGATGGTTGGACAGATTTCTCGTTGGACGACGATGTT GATTTGCGTAGTCGTGTTCCATTTTCTGTCTACATGTTGATGAAGGAGTGTGTTTGTGTGTGTGTGTGTGTGTGAATGTGGGATA TAGTGTGTTGGGTTGATTATGTGTTGATTGTTTTTCGTTGTAATCGTGGTGTTTGAATGTTTGGTGAATGATGGTTATGGGGAGG AGTGGTGTTCCCTGTTATGTTTCCGTTGTTCAGGTTGTGTGTGTTGTGTGTGTTGTGTGTGTTGTGATGTTCTAGTGTGTGTAAT GCATGTAAGTACTCTCAACCAGTCCACTTGTTGTTTGTTAACATATGTGTGGGTGTAATGAGAGTGACATTGATGGTTGGACAGA TTTCTCGTTGGACGACGATGTTGATTTGCGTAGTCGTGTTCCATTTTCTGTCTACATGTTGATGAAGGAGTGTGTTTGTGTGTGT GTGTGTGAATGTGGGATATAGTGTGTTGGGTTGATTATGTGTTGATTGTTTTTCGTTGTAATAGTGGTGTTTGAATGTTTGGTGA ATGAAGGCTGTGGGTTTCTTTGTTTTCTTGTATTTAGTTCTGATTTTATTTCTGTTTGTCTTTTTTTTATGTTTGCGGAGTGTTT TCTTTTTTGTATTTTTTTTGGTCTCTAATATTGTTATTAATTTATTTTCTTTTTTCGTGTTTTATTTGTTCTCTCTTTTAGGTT GATGATGAAGATTACATTCCTTCTCGTTCTGAGCCATCTGATGTGAAGTACAATCGTCGTCGTTTGCTTATTCTTGCTCAAGTGG AACGTGTATGTTTTTTTCTATTGTATAGTACTTGTTATATATATATTTTTTATCGTGTTTGTGTCGTAGATCGTTTTTCATTTGT TGTAACTTTGTAGGTCAAAAATGATCCTCTCTCCGTCGTTTTATTCGTTGCTTTGTTAATTACACTTTTGACTTTTCTTTTACGA TTGTTTCTATTTTTTTGACAGATAATTCCCTTTTAAACATTCGAAATTAAAAATACGAAGTTGAGCGAAGAGATCTCATTTCAAC GAATTTTTATCAAGCTGTACAATCGTACTAATATGGAAAATTGTTTTTTTGTTAAAGTACTAATGGGAACACAAATAACCGAGA TGATGGAAAATAATCTTTCTATTGAATCATCTTGGTTATTCGTGTGCACTTTAAGGGCTGAATTTATAACAGAAGTTTGCTGAAA CGGCCTCAAATCATTTAGTTTTTCCCTTGTTAATAAATGGCAGTTCTGATAATTAACATAACCCATTGTGTTATTGGATAAGAAT 
AAAGAGAAGTCAGCGAAGAAAATTTTCTTTTCCACTAAATCGTTTACTTAAGCTGTACATTCTTGTTTATAATCATACGGAAAAT ACATGTCTAGAGAAGGATAGAAAAGATTGCATTATAAATGGATTCGAGACTCTTATTATCAAATGAGGTTTCGCAATAACCAATG GGTAAGGAAGGAATATAACGATGAAAATAATAATTCACGGGTCAGATAATTGTCCAATTGACAGGTATGAAGAAAAATGACAAAC ATGAAGGTCATAATAAAAACTGAATAATAATCATGAAAACTTGTAATAATAATAATAATAATAACTGATTAGAGTTTAAAATTAC AAAACAGTTATCTAAAAAATCAATAATAACCGAAGAAAAACGAAGAAAAAAGGGAGAAAAACCGAAACATACTACTAAAGAGAAT CAGGGGCAAGGAAGGATGAGAGGGGTTGGACCAGTCGTTTCTGTACACAAAGTTCGGGTCTGAGTTCGTGGATTGCCAATGCTCC GGCAATGCATAAAATATTGATTCAATTTCCCTTCGATCCAATTCATTTGACTGTGTAGATTACTTTATGAGAAAACTCTTTTGCA GTGATGTGTTCACAGTTAATTAAATGCTCCTGTATAGAACTAGTAATTGTTTTACATTCTCCTTTTAAAAGCCATGTCGGATAGT GTTCTGAGATGCGTTTTGGAAAGTGCTCGCTTTGTGCGGCCAATGTAGCCTGCCCCACACTAGCAAGTAAATTTATAGATACACG TTGATGTGGCCCAAAGAGGAAGTTTATACTTAACACATCGGAGAATGGTGGGCCGGCTAGAGAAAACAATTCTGAGCTTAGCTGA GTAGAACGTTTTATTCAACGATTTTGAAATACGTTTATTTAAGATTTCAGCAGTCGTATCTCCCTTAAATTCCATATTCATAAAT AGAATTTTCTTTTGTACTGTTGGTGCTTTGTCAGGATGACGTCTAGCTGTTAAGTTGCTACCGACGAATCTAGGTGGATAACCAT TCCCAAAGAGAATCTGTCGACGTCGAAGTAGTTCCTCATCTATTGTGTCAGTAGGACATACTCGCCTGATCCTTGAGGATAAAGA GTGAATCAAGTTCCTCTTTCTACTCAGTGGTACCCGGCTATGGAAATTCGTTTACTGCCCATTCCAGGTCGCTTTTCTATACATC AAAAAAAAGAAATTAATTGTTTGCCTCTGCTTCCAAAGTGAATTGAATAGAGGGGTGATAATTACTGAAACTTCAAAATTTCATT GAGGTTTATATCTTCTTCACAAATAATGAAAGTATCATCCATAGATCACCTATATAAGTGAAATCTCTCAATTATTGGTCGAAGT TAGGTATTTTCTAGATTGGCCATGAAACAATCAGCCAAGAGGAGAACCAATAGAGAACCCATTGCAACTCTATCCATTTGTCTGT AGGAATCATCATTAAATCTAAGTTGCACGTTGAGAGTACTTCGTAGAAGTAGCTCTTCTAGATAATGTTTTGGGATACAAATATC AAGGTTATTGTTTTCAATATAGTTACATAAAAAACATAGTTTCTGTGAGAGGAACATTTGTAGAAAGAGAAGTAACATCCAGTGA AATCATTTTCCTATCATTTATATTGACATTTTTAATTGAGTCGACAAAGTCAAAGGAATCAAGAGAATTGTATTTATTGATATGA TTTTTAACGGATTGTAAAATATCAACTGGCCATTTAGCAATCAGATGATACAGAGAGTCGTCCATATCGAGCATAGGATGTAGAG GGACATTATAACTATGAACTTTTGTGAGTCCATATAGTCTTGGTATAACGGAACCCGATGGTCTAATATGTTCATAGAGGTCACA ATTGATAGTAAACTCATCTTTCATCTTCTTTAGGATTTTAATAAGCATCTGTTCCGTTTTTTTCAGTTGTCTTTTTGAACGGTTT CTTTGGAGAATTTGACAGGATCATATAATATCGAACACAATTTGGTGACATAGTCAAGACGATCCACAAAGACGATTCTCAGACC CTTGTCTAGGAGACACAACACTAAATCCTGATTTTTCGGAAGATCAGATAATTGAGATCTATGCTCTTTTGTGAGTAATCGACTA ATTGTAGGTATTTTCTTCACATATTGATAACAACAGTCTACAAGAGTTGTTTTGAAATGCTCATAATCTTTATTAGATACTGGAA TAAAGTCACTTGTCTGATGAATGAGGCTTTCAACCTAGATTTTAGCGTTGAGTTCATTAATGTTAGTTGAAGTACTACAAAATTT AGGAACAACATTTATCTATAAATTTACTTGCTAGTGTGGGGCAGGCTACATTGGCCGCACAAAGCGAGCACTTTCCAAAACGCAT CTCAGAACACTATCCGACATGGCTTTTAAAAGGAGAATGTAAAACAATTACTAGTTCTATACAGGAGCATTTAATTAACTGTGAA CACATCACTGCAAAAGAGTTTTCTCATAAAGTAATCTACACAGTCAAATGAATTGGATCGAAGGGAAATTGAATCAATATTTTAT GCATTGCCGGAGCATTGGCAATCCACGAACTCAGACCCGAACTTTGTGTACAGAAACGACTGGTCCAACCCCTCTCATCCTTCCT TGCCCCTGATTCTCTTTAGTGGTAGTTCTGATTTTCAAGGTAGCCCACTCTAATAACTTTCCAATTCATATTTGCTTACTGATCC TTGTTGTTATTTGATTGATTGTTCTCGTTAGGTATTTTTTATCAAATGTCTCAAGTCCATTTTCTTGATTATTAACAGAATTGTC CCATTAAATTTTCGATTTCATCGAATGACAATTTTTTTCCTGAGTTCTACACTTTTTCTCATCAATCAAGGATTATTCTTGAACT ATCGTGTATATGTCGTACGGATTTGTTCGCGTGAATTGCATTTCGCCCTAAAAACAGCTTGCTGTCGTTCGTAATTCTTAATTAT CATAGCTTTCACAAAAGTGAACGAGACTGTTGGGAAGCTTTGAAATTATGTAAATAATATCATTATATACATTATCTATATATAT ATGAAGATGGTTATTTATAGTGTTTAGTGATTTTTATGTTTTCTCTATTTTTTCCTCTCATATCTGTTTAGATGTTTTCAATATT ATTGATCTTAGATGAAGTTTCCTTATCTCTCAAAAGAGTTGTTGTTCAAAATGACGCGTAAGTGTACCCTATACTTTTTGTAGAA CTATGACTTTTTGACTTTCGGGTCTGATTTTTCACTTGAGAGCTGTACTGTTTTGTATTGTATCGCTCTAAAGTTCCCGTTTATA ATCCAAAACGTGAAAACATTTAAATAAAACACTCGAAAAATAAATCAAACCTAGTGACCAAATATGCAATGAAGACCATTCATAT CACGATTCTGTTCCAGAAA GTAATAAAATCCAGGGTTTTATCACTGCGAAAT GATCAGTATTGAACCCTTTAAAATCTTTAAAAT TATTAGAGTGTTTGAGGTCAGTGTTTCAATACGTATATTTATTCATGGAAATGTCATCTACATGATGGTTGAGAGTGTATCATAT TATTACATCATTACTAGTAAGTTAATTCTACATTTGTTCCAGGTCAATCCATTCTTCTAGATATTCTTTAAAATTGTTAAAAGGC TTGATATTTACCCACCTCAAAAAACATTCCACTATTATTCGCTACTATTAAATTAGATAATCTGCATCTGCTTTTAGAAAAAGCT ACAGGATTCTGTCACTTCCATCTATTTAAACCTTAATTATTACTAATATCCCTAATATCTTATATGAATTTTTGTGCAAGTTTTC ATGTCATGGACCTAGGCATACAAAAGTTTTATAATTCAATAAAACAGAACGATCTTTAAGAAATAGAAACTCCACAATTGCATTG TGTTTATAGGTGTTGCTTTGACGTTTTGTGAATTCGTATACTACTTGGATAGTTTTTTTCTTCTTCATAGTATCTTGTGACTTCA ATTAATGTTATTCCAAACTTTTCGGCATATTGACAAACATATTTGAATATAGAGCCTATTTGGATTTATTTTTTACAAAAAAATA TAAAAAGTATTTGTGTATATATTCCTTGATTTGATTTCTTCTTCCCATAGACGTTCTCGACTGTTAGATTATCAACAAGAATTGA TTAATCAACTAATCA

\section{Alignment: SmBr18/EST Contig/scaff000011/scaff000068 showing contig sequence}

$\operatorname{SmBr} 18$ scaff000011 EST Contig scaff000068 Contig-0

$\operatorname{SmBr} 18$ scaff000011 EST Contig

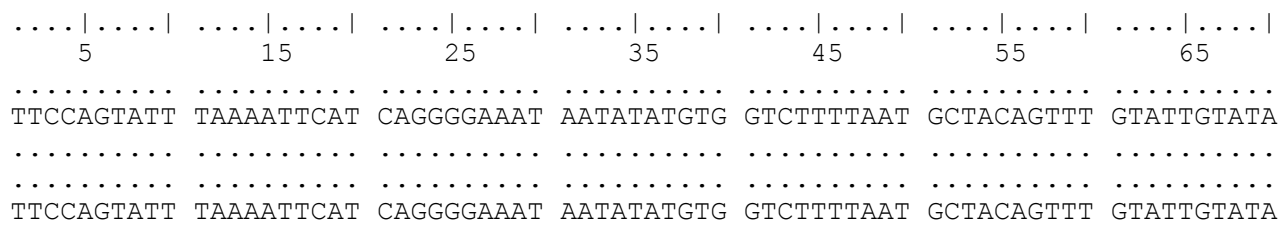

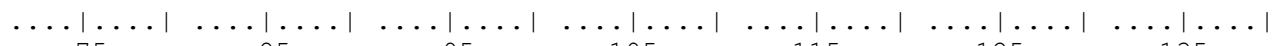
75 85 95 115 135

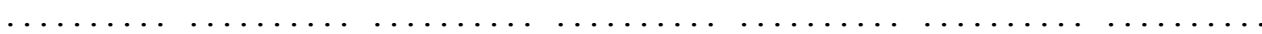

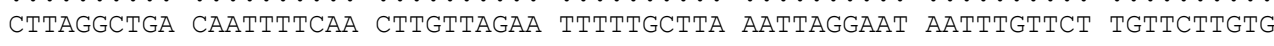

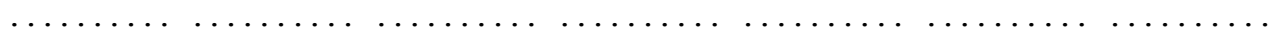


scaff000068 Contig-0

SmBr 18 scaff000011 EST Contig scaff000068 Contig-0

SmBr 18 scaff000011 EST Contig scaff000068 Contig-0

SmBr 18 scaff000011 EST Contig scaff000068 Contig-0

SmBr 18 scaff000011 EST Contig scaff000068 Contig-0

SmBr 18 scaff000011 EST Contig scaff000068 Contig-0

SmBr 18 scaff000011 EST Contig scaff000068 Contig-0

SmBr 18 scaff000011 EST Contig scaff000068 Contig-0

SmBr 18 scaff000011 EST Contig scaff000068 Contig-0

SmBr 18 scaff000011 EST Contig scaff000068 Contig-0

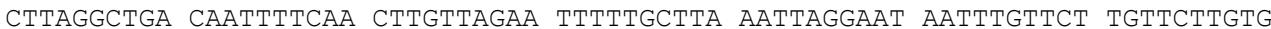

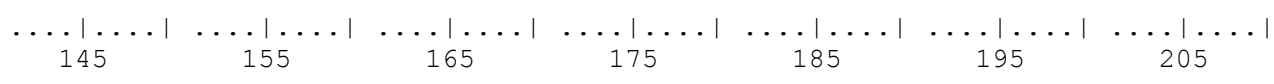

GTTGTGTGGT TTCATTCAGT TTCATATATA TCTATTCTTT ATTGGACTGA GCTTCAACCT TGCTAGTCGA

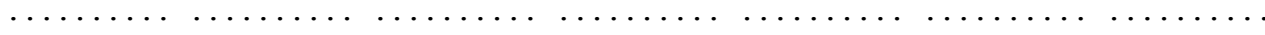
GTTGTGTGGT

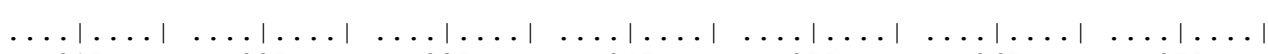
215
225
235
245
255
265
275

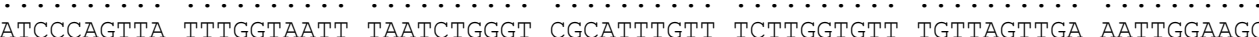

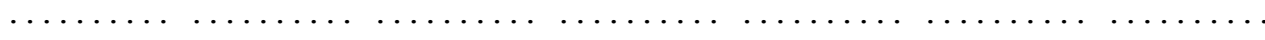
ÄCCCAGTTA TTTGGTAATT TÄATCTGGGT CGCATTTGTT $\ldots|\ldots| \ldots|\ldots| \ldots|\ldots| \ldots|\ldots| \ldots|\ldots| \ldots|\ldots| \ldots|\ldots| \ldots|\ldots| \ldots|\ldots| \ldots \mid$
285
295
305
315
325
335
345

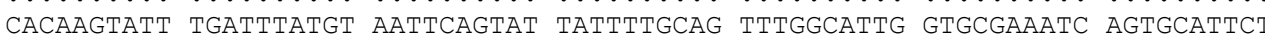

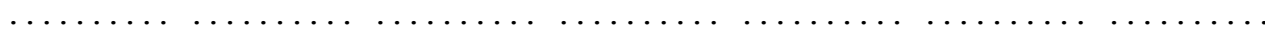

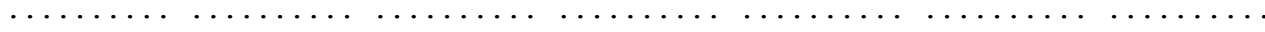

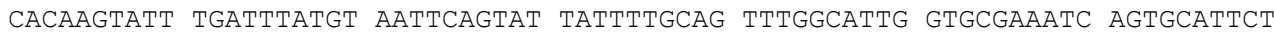
$\ldots|\ldots| \ldots|\ldots| \ldots|\ldots| \ldots|\ldots| \ldots|\ldots| \ldots|\ldots| \ldots|\ldots| \ldots|\ldots| \ldots|\ldots| \ldots \mid$
355
365
375
385
395
405
415

TCAACAAÄं

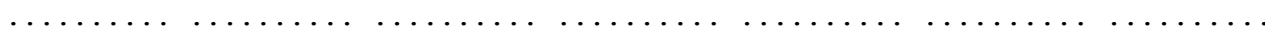

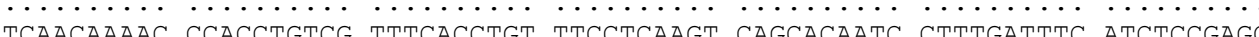
TCAACAAAAC CCACCTGTCG TTTCACCTGT TTCCTCAAGT CAGCACAATC CTTTGATTTC ATCTCCGAGC

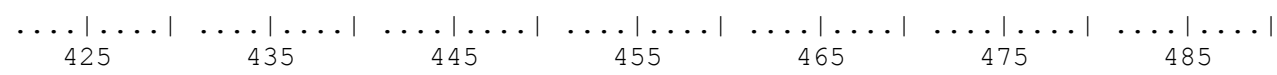

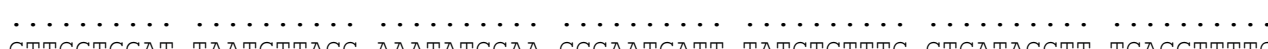

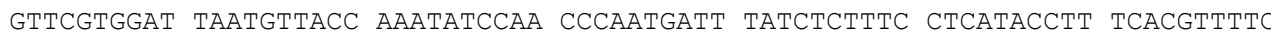

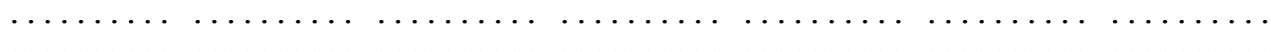
GTTCGTGGAT TAATGTTACC AAATATCCAA CCCAATGATT TATCTCTTTC CTCATACCTT TCACGTTTTC

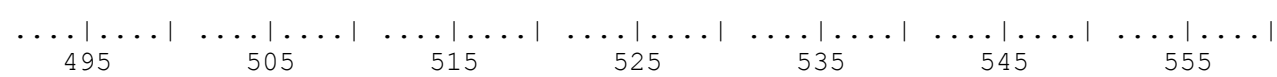

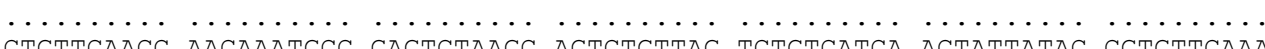
CTGTTCAACC AACAAATGCC GAGTCTAAGC AGTCTGTTAG TCTGTCATCA ACTATTATAC CGTGTTCAAA

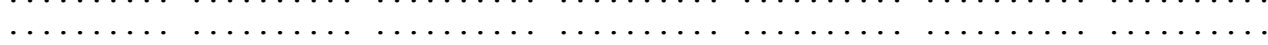

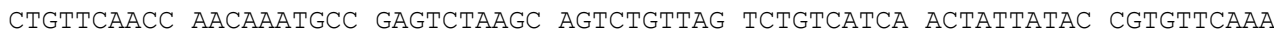

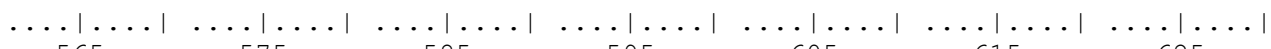

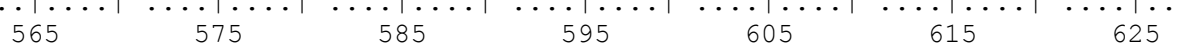
ACAACCATCA GGATTCGTCG

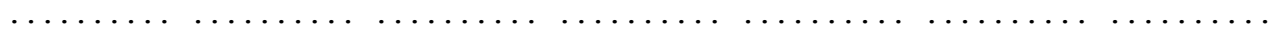
ACAACCATCA GGATTCGTCG CTTCACTCGA GGAAgTgTCC ACAGTTAAAG AAAGCGACCC AAACCGTGGT

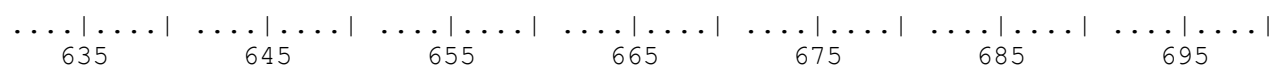

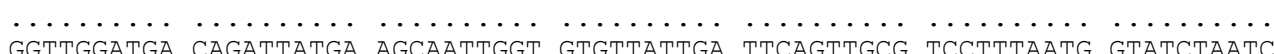

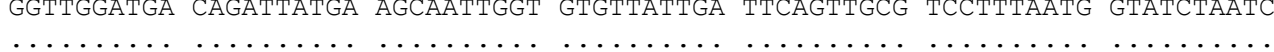

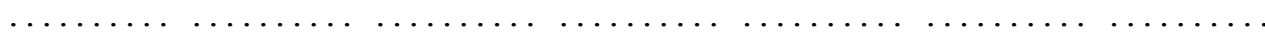

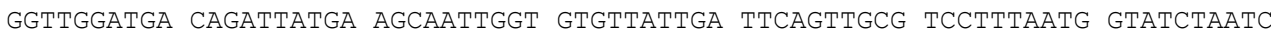

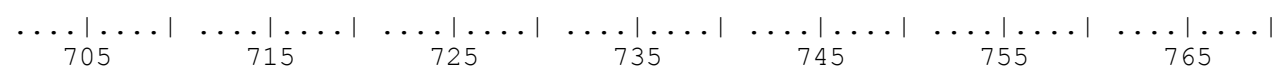
CCTATGTCCA GGTCAGTTTC TTATTTTCTG AATGAGTTTC TAATTTACTA C̈ACTTTTAGT TGATTATGTG

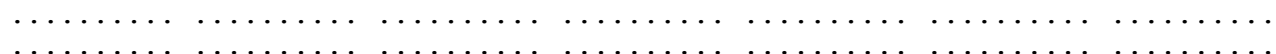

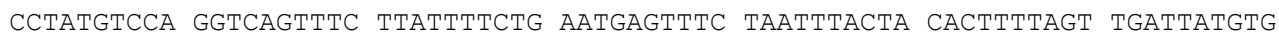


$\operatorname{SmBr} 18$ scaff000011 EST Contig scaff000068 Contig-0

$\operatorname{SmBr} 18$

scaff000011 EST Contig scaff000068 Contig-0

$\operatorname{SmBr} 18$ scaff000011 EST Contig scaff000068 Contig-0

$\operatorname{SmBr} 18$ scaff000011 EST Contig scaff000068 Contig-0

SmBr18 scaff000011 EST Contig scaff000068 Contig-0

SmBr18 scaff000011 EST Contig scaff000068 Contig-0

SmBr18 scaff000011 EST Contig scaff000068 Contig-0

SmBr18 scaff000011 EST Contig scaff000068 contig-0

SmBr18 scaff000011 EST Contig scaff000068 Contig-0

SmBr18 scaff000011 EST Contig $\begin{array}{rrrrrrr}775 & 785 & 795 & 805 & 815 & 825 & 835\end{array}$ 5 ATTCTTAAGT AGCAGAGTTG CTTGAACATT AATGCCTCTG ATCACTGAAT GATTTTGTCG GCTGGATCGT

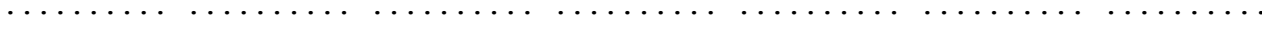
ATTCTTAAgt AgCAGAgtTg CTTGAACATt AATGCCTCTG ATCACTGAAT GATTTTGTCG GCTGGAtCGt

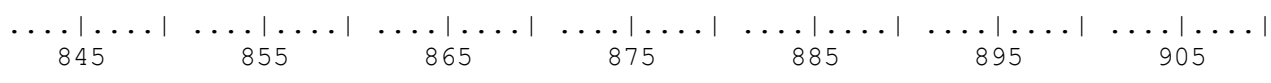

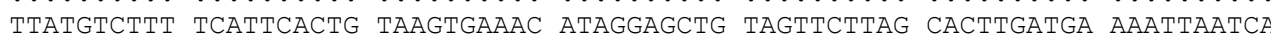

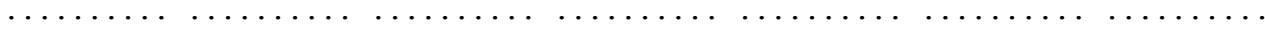

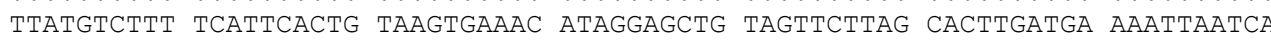

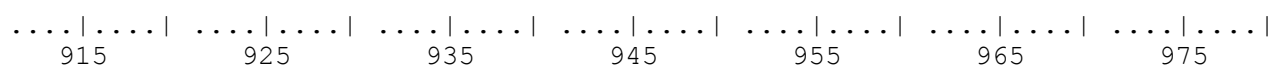

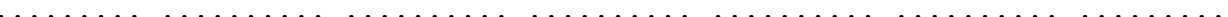

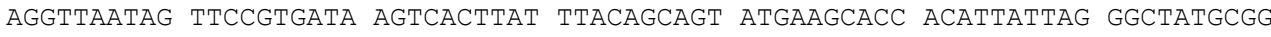
$\cdots \cdots \cdots \cdots \cdots \cdots \cdots \cdots \cdots \cdots$

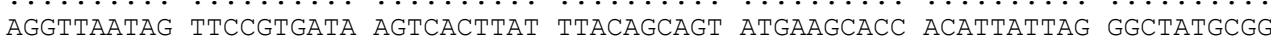

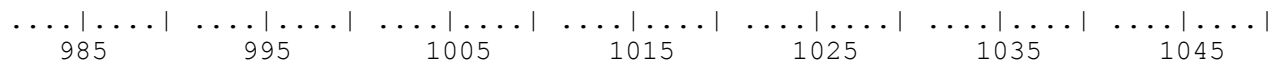

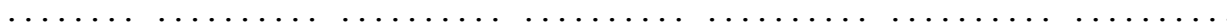
TAATAAAAAT GTAGGTCAAA CCTCTCCATA TATCTGTTTA GTGGATATAA TTTGGGCGAA ATTGCCTGTT

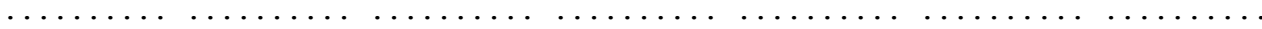
TAATAAAAAT GTAGGTCAAA CCTCTCCATA TATCTGTTTA GTGGATATAA TTTGGGCGAA ATTGCCTGTT

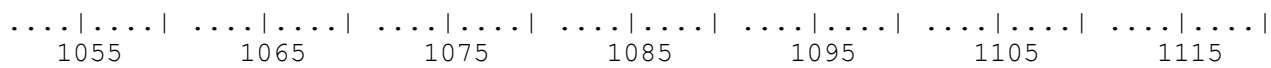

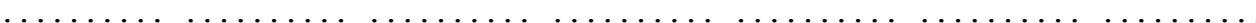
TTGAAACGTC TTTTAATACA GTTTGAAAgT TGATACACCA TATATTTATC ATCAAAATGA TGGAATTCGA

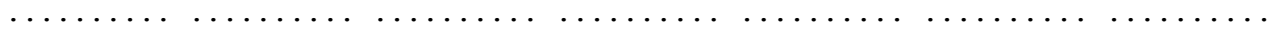
TTGAAACGTC TTTTAATACA GTTTGAAAGT TGATACACCA TATATTTATC ATCAAAATGA TGGAATTCGA

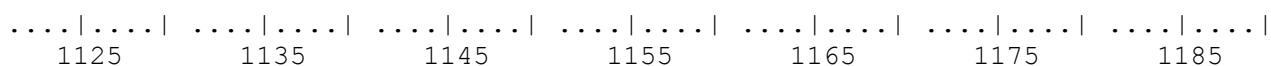

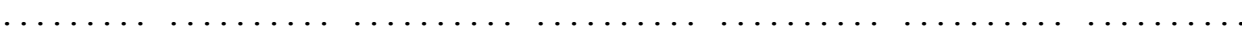
CATCAAAATA CTCGTTCTTT GAATtTCAgG TTCAATGAAA TCCCACTTGT TTCGGATGAT TTCCTTCAAg

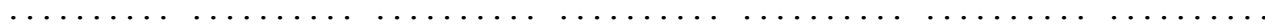
CATCAAAATA CTCGTTCTTT GAATTTCAGG TTCAATGAAA TCCCACTTGT TTCGGATGAT TTCCTTCAÄ

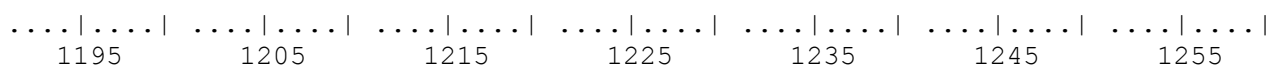

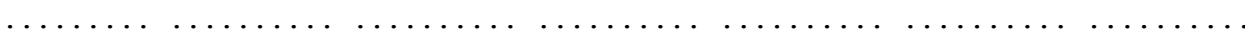
AATAATTTGC TATAACTTCT GAGGATTTTG GCACAGTTGA TAGTTTTTTC AACGGTTAAT TGTAATAAAT

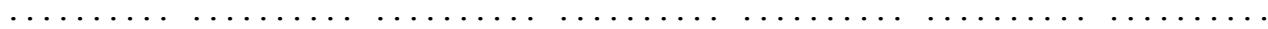
ÄATATTTGC TATAACTTCT GAGGATTTTG GCACAGTTGA TAGTTTTTTC ÄACGGTTAÄT TGTAATAAAT

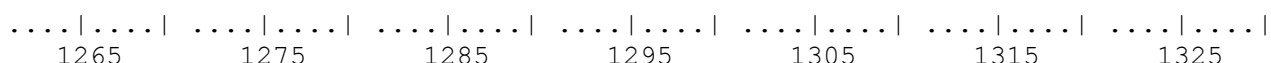

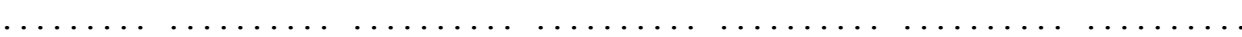
AgtTGTCTAT TTATCTCAAA TTATGTATTC AAATTTCACA TAACAAATAA TCAAATTCTA ACAAACAATt

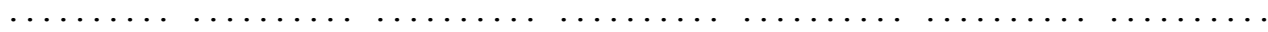
AGTTGTCTAT tTATCTCAAA tTATGTATTC AAATTTCACA TAACAAATAA TCAAATTCTA ACAAACAATt

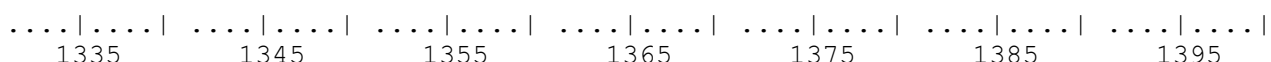

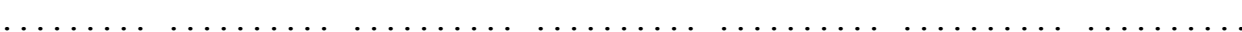

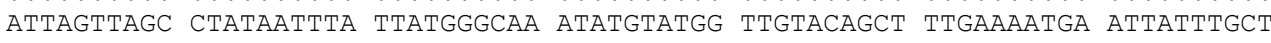

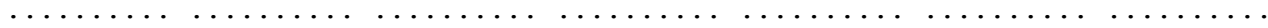

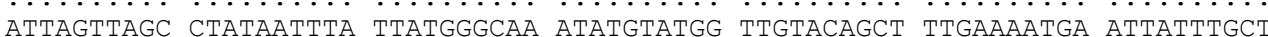

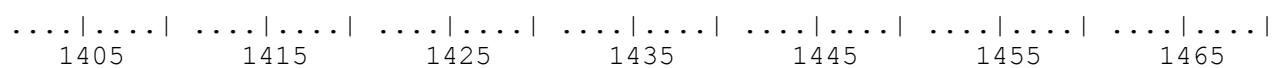

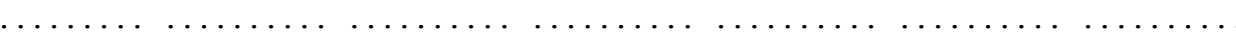

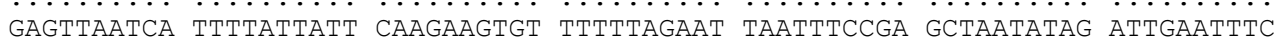


scaff000068 Contig-0

SmBr 18 scaff000011 EST Contig scaff000068 Contig-0

SmBr 18 scaff000011 EST Contig scaff000068 Contig-0

SmBr 18 scaff000011 EST Contig scaff000068 Contig-0

SmBr 18 scaff000011 EST Contig scaff000068 Contig-0

SmBr 18 scaff000011 EST Contig scaff000068 Contig-0

SmBr 18 scaff000011 EST Contig scaff000068 Contig-0

SmBr 18 scaff000011 EST Contig scaff000068 Contig-0

SmBr 18 scaff000011 EST Contig scaff000068 Contig-0

SmBr 18 scaff000011 EST Contig scaff000068 Contig-0
GAGTTAATCA TTTTATTATT CAAGAAGTGT TTTTTAGAAT TAATTTCCGA GCTAATATAG ATTGAATTTC

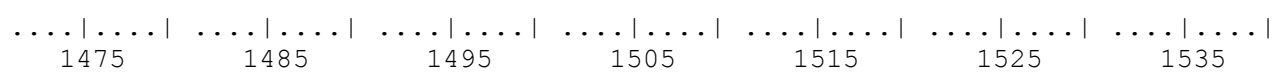

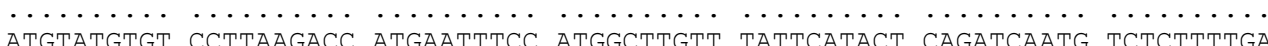

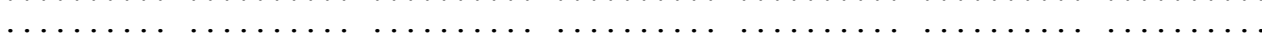

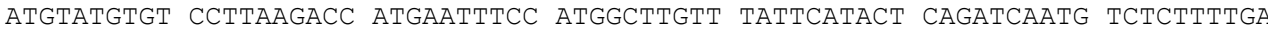
$\ldots|\ldots| \ldots|\ldots| \ldots|\ldots| \ldots|\ldots| \ldots|\ldots| \ldots|\ldots| \ldots|\ldots| \ldots|\ldots| \ldots|\ldots| \ldots|\ldots|$
1545
1555
1565
1575
1585
1595
1605

TTTTGAACTT TCTATGTAAA AGTGACCAGG AACTTCCGAG GCTTATTCAT TTCTATCTTG AATAACCGAA

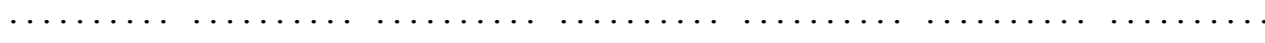
TTTTGÄСТ $\ldots|\ldots| \ldots|\ldots| \ldots|\ldots| \ldots|\ldots| \ldots|\ldots| \ldots|\ldots| \ldots|\ldots| \ldots|\ldots| \ldots|\ldots| \ldots \mid$

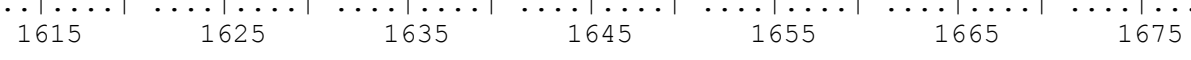

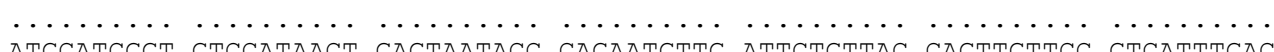
ATGCATCGGT GTCCATAACT GAGTAATACC CAGAATGTTG ATTCTGTTAC CAGTTGTTCC GTCATTTGAC

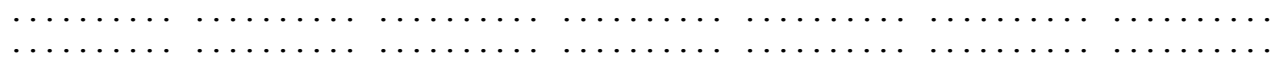
AtgCATCGgT GTCCATAACT GAGTAATACC CAGAATGTTG ATTCTGTTAC CAGTTGTTCC GTCATTTGAC $\ldots|\ldots| \ldots|\ldots| \ldots|\ldots| \ldots|\ldots| \ldots|\ldots| \ldots|\ldots| \ldots|\ldots| \ldots|\ldots| \ldots|\ldots| \ldots \mid$

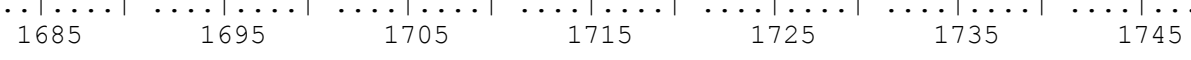

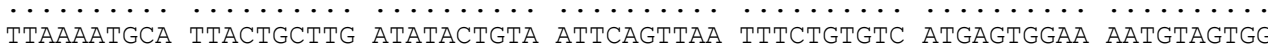

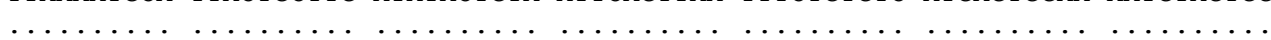

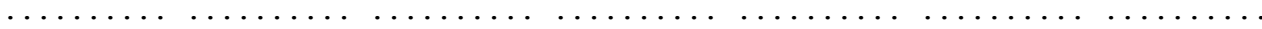

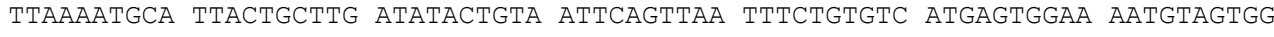
$\ldots|\ldots| \ldots|\ldots| \ldots|\ldots| \ldots|\ldots| \ldots|\ldots| \ldots|\ldots| \ldots|\ldots| \ldots|\ldots| \ldots|\ldots| \ldots \mid$

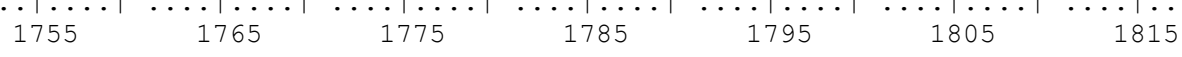
TTGTTTTATT CAGACAAATT TTTAGTTGTT AGTTTAACGG TATAGTAGTT TCTCCATTTC GAGACATAAT

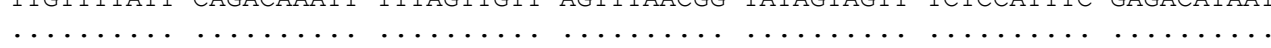

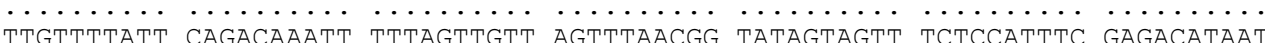
$\ldots|\ldots| \ldots|\ldots| \ldots|\ldots| \ldots|\ldots| \ldots|\ldots| \ldots|\ldots| \ldots|\ldots| \ldots|\ldots| \ldots|\ldots| \ldots|\ldots|$
1825
1835
1845
1855
1865
1875
1885

CTCACTGTTA TAGTTGTTTT TAATCTCCCA GCTATACAAC AAAATAAAAA TAAATAACAT CCGAAAATTG

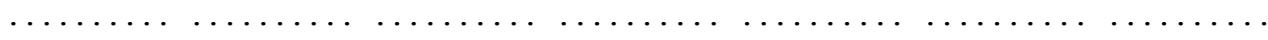

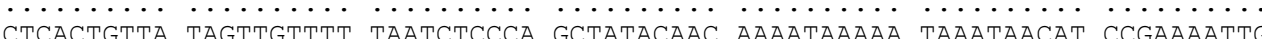
$\ldots|\ldots| \ldots|\ldots| \ldots|\ldots| \ldots|\ldots| \ldots|\ldots| \ldots|\ldots| \ldots|\ldots| \ldots|\ldots| \ldots|\ldots| \ldots \mid$ $\begin{array}{ccccccc}1895 & 1905 & 1915 & 1925 & 1935 & 1945 & 1955\end{array}$ GCTAATTTCT AAGTTTGAAT TTTGTTTAGG TATACGTTTA TTGCTGTCAA TGTACCTGAG CGTCTTTCTT

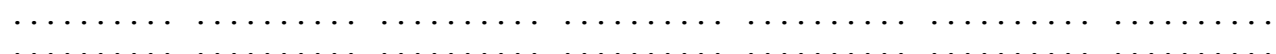
GCTAATTTCT AAGTTTGAAT TTTGTTTAGg TATACGTTTA TTGCTGTCAA TGTACCTGA CGTCTTTCTT

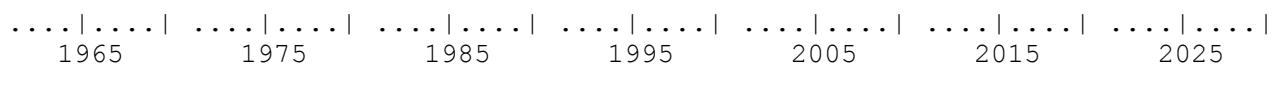
TTTGTCGTGA ACAAGTCTAA TAATGATATC GTACACTGAC TAGTTATTCA AGGACTACTA ACCATTATTT

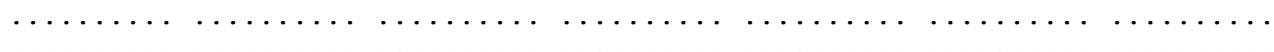
TTTGTCGTGA ACAAGTCTAA TAATGATATC GTACACTGAC TAGTTATTCA AgGACTACTA ACCATTATTT

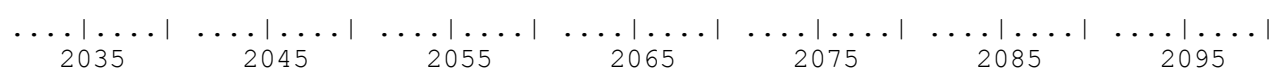

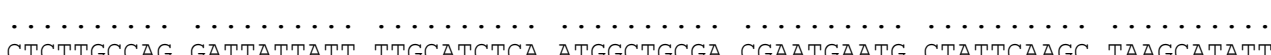

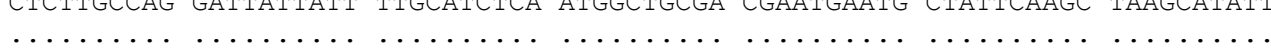

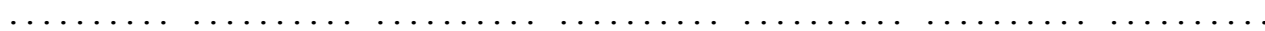

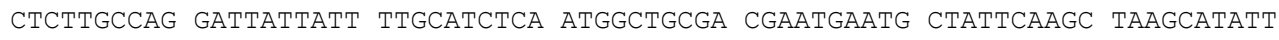
$\ldots|\ldots| \ldots|\ldots| \ldots|\ldots| \ldots|\ldots| \ldots|\ldots| \ldots|\ldots| \ldots|\ldots| \ldots|\ldots| \ldots|\ldots|$ 
SmBr 18 scaff000011 EST Contig scaff000068 Contig-0

SmBr 18 scaff000011 EST Contig scaff000068 Contig-0

SmBr 18 scaff000011 EST Contig scaff000068 Contig-0

SmBr 18 scaff000011 EST Contig scaff000068 Contig-0

SmBr 18 scaff000011 EST Contig scaff000068 Contig-0

SmBr 18 scaff000011 EST Contig scaff000068 Contig-0

SmBr 18 scaff000011 EST Contig scaff000068 Contig-0

SmBr 18 scaff000011 EST Contig scaff000068 Contig-0

SmBr 18 scaff000011 EST Contig scaff000068 Contig-0

SmBr 18 scaff000011 EST Contig
2105
2115
2125
2135
2145
2155
2165 .

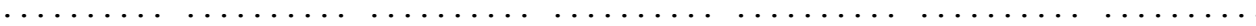
TCAAAACATA AAATGAACAC TACAACTTCA CCAGCAATAG TGCAgCtTCC TATtCCtAtA CCATtGgAAA $\ldots|\ldots| \ldots|\ldots| \ldots|\ldots| \ldots|\ldots| \ldots|\ldots| \ldots|\ldots| \ldots|\ldots| \ldots|\ldots| \ldots|\ldots|$
2175
2185
2195
2205
2215
2225
2235

CCTAGCGA TTCCAATCA T-CATATCAA ............ ... TTA TTCATCTC-- G-AA-T-TCA AACACGA-CT --ATATTACA GT---GAG-T GCTTA $\cdots \cdots \cdots \cdots \cdots$ $\ldots|\ldots| \ldots|\ldots| \ldots|\ldots| \ldots|\ldots| \ldots|\ldots| \ldots|\ldots| \ldots|\ldots| \ldots|\ldots| \ldots|\ldots| \ldots|\ldots|$

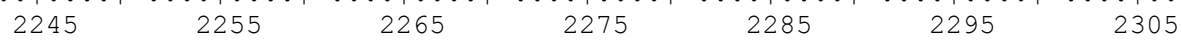

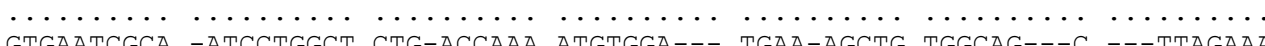
-TGAATTG-A GA----GG-T GTGTAGAAAA ATA-GGACAg TGGAGAG-TG TGACACAAAC AAATTAGAAT

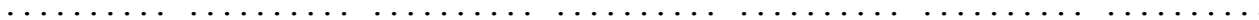
GTGAATCGCA GATCCTGGCT CTGTACAAAA ATATGGACAG TGAAGAGCTG TGACACAAAC AAATTAGAAA

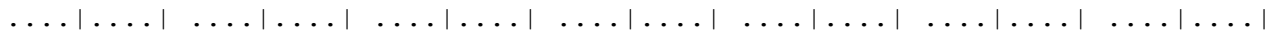

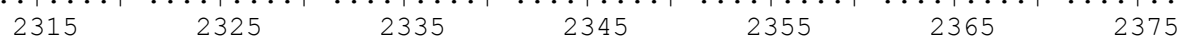

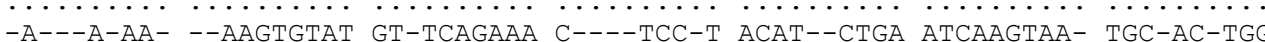
GATCCACAAC TCAACTGG-T GCCTCAGTAA CCGCCTCCAT A-ATGGCTGG GTTT-GTAAG TGCCACCTTT

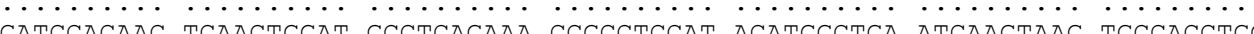
GATCCACAAC TCAACTGGAT GCCTCAgAAA CCGCCTCCAT ACATGgCtGA ATCAAGtAAg TGCCACCTGG

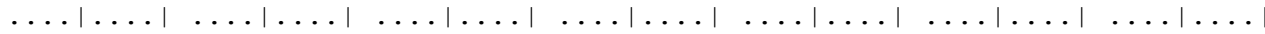

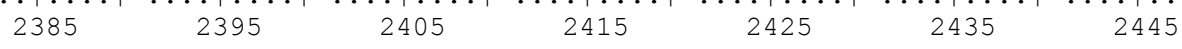

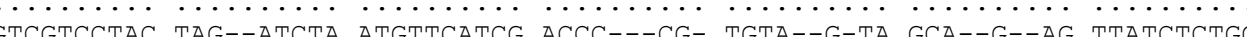
GCCG-CCTT- T-GCCACCTT -TGC-CACC- ACCCTTACCC T-TACCGCTA CCACCGCCA- TAACC-CT-

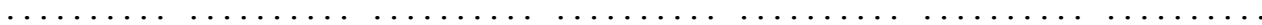
GCCGTCCtAC tAgCCACCTA AtgCtCACCG ACCCTtACCC TGtACCGCTA CCACCGCCAg tAACCTCtgG $\ldots|\ldots| \ldots|\ldots| \ldots|\ldots| \ldots|\ldots| \ldots|\ldots| \ldots|\ldots| \ldots|\ldots| \ldots|\ldots| \ldots \mid$ $\begin{array}{lllllll}2455 & 2465 & 2475 & 2485 & 2495 & 2505 & 2515\end{array}$

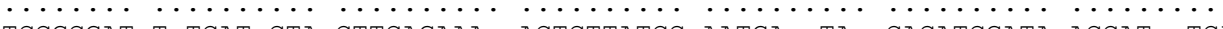
CGTCCGCGAT T-TCAT-CTA CTTCAGAAA- ACTCTTATCC AATGA--TA- CACATCGATA ACCAT--TCA T-TCCATAAT TGT--TGC-- CTTTACTACC AC-CTTTTCC AAAGCCGTTG C-CACCGTTA -CCATAATCA $\ldots \ldots \ldots \ldots \ldots \ldots \ldots \ldots \ldots \ldots \ldots \ldots \ldots \ldots \ldots \ldots \ldots \ldots \ldots$ CGTCCACAAT TGTCATGCTA CTTCACAAAC ACTCTTAtCC AAAgACGTAg CACACCGAtA ACCATAATCA $\ldots|\ldots| \ldots|\ldots| \ldots|\ldots| \ldots|\ldots| \ldots|\ldots| \ldots|\ldots| \ldots|\ldots| \ldots|\ldots| \ldots \mid$ $\begin{array}{lllllll}2525 & 2535 & 2545 & 2555 & 2565 & 2575 & 2585\end{array}$ $\cdots \cdots \cdots \cdots$ CCGCCATAGG GGGCAGGGAA GGCCTCATCG AAGCCGgGGC GAGGGTG-T- CAAAAAAGCA GGGTCCACCA

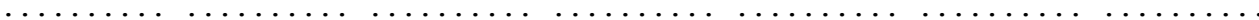

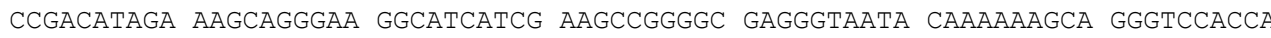

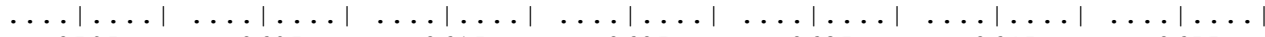

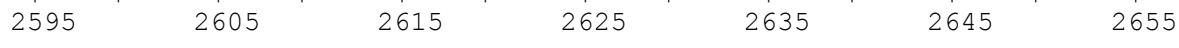

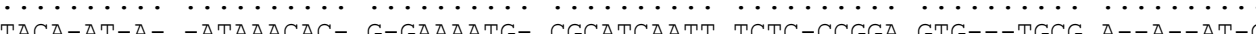
TTCCCACCAC CATAGCCACC GCGGTAATTA C-CA-CAATC TC-CGCC--A TTGCAAT-C- ACCACCATAG

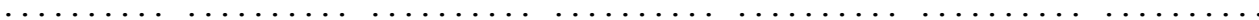
TACACACCAC CATAAACACC GCGAAAATGA CGCATCAATC TCTCGCCGGA GTGCAATGCG ACCACCATAG

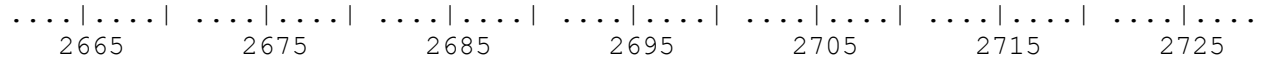

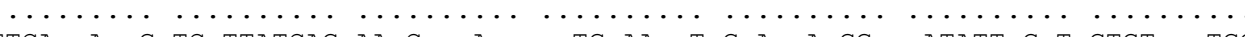
TTCA--A--G TC-TTATCAG AA-C---A-- --TG-AA--T G-A--A-GG- -ATATT-G-T GTGT---TGG C-CACCACCG TAATTACCAC AATCTCCACC ACTGCAACCT CCACCATGGC CATAACCGCT ATCACAATCG

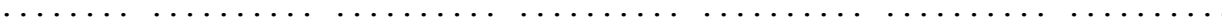
CTCACCACCG TAATTACCAC AATCTCCACC ACTGCAACCT CCACCATGGC CATAACCGCT ATCACAATCG

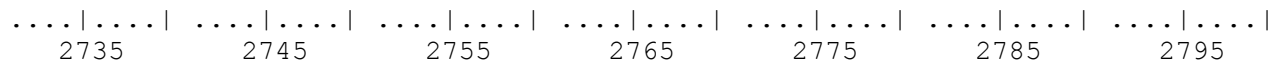

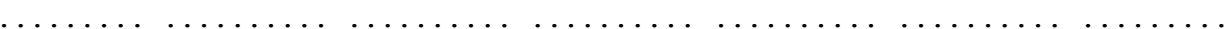
GTTGATTATA TGTT---G-- ATTGTTTTT- CGTTGTAATA GTGGTGTTTG AATGT--T-T -GG---TG-CT--ACCATA -GCAACCGCC ACCGTACCCA CCC-G-AATA GTCATG--TG A-TGTGGTGT AGGCGGTGGC 
scaff000068 Contig-0

SmBr18 scaff000011 EST Contig scaff000068 Contig-0

SmBr 18 scaff000011 EST Contig scaff000068 Contig-0

SmBr18 scaff000011 EST Contig scaff000068 Contig-0

SmBr18 scaff000011 EST Contig scaff000068 Contig-0

SmBr18 scaff000011 EST Contig scaff000068 Contig-0

SmBr18 scaff000011 EST Contig scaff000068 Contig-0

SmBr 18 scaff000011 EST Contig scaff000068 Contig-0

SmBr18 scaff000011 EST Contig scaff000068 Contig-0

SmBr 18 scaff000011 EST Contig scaff000068 Contig-0
CTTGACCATA TGCAACCGCC ACCGTACCCA CCCTGTAATA GTCATGTTTG AATGTGGTGT AGgCGGTGGC

$$
2855 \quad 2865
$$

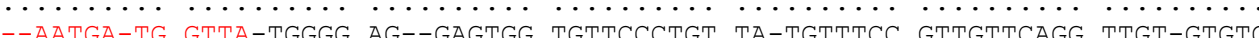
GTAACCAATG GCTACTAAGA AGACGAGTG- TGAG---TG- -ACTGTTTC- GTTTTTCAAA TTGTTG-GTG

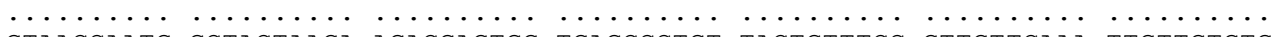
GTAACCAATG GCTACTAAGA AgACGAgTGg TGAGCCCTGT TACTGTTTCC GTTGTTCAAA TTGTTGTGTG $\ldots|\ldots| \ldots|\ldots| \ldots|\ldots| \ldots|\ldots| \ldots|\ldots| \ldots|\ldots| \ldots|\ldots| \ldots|\ldots| \ldots|\ldots| \ldots \mid$
2875
2885
2895
2905
2915
2925
2935

.T AGATGCATGG TAAGTA CATC TACAACCAAG ITGTGTGTGT TGTGTGTGTt G-TGATGTTC TAGTGTGTGT A-ATGCATG- TAAGTAC-TC T-CGACCA-G TTGT-TGTAC TG-G-GTGT- GATGATGTTC TAGTGTGTGT A-ATECATG- TAAGTAC-TC T-CAACCA-G

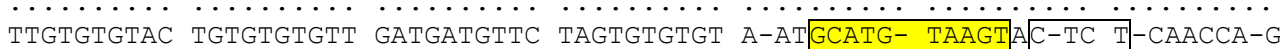

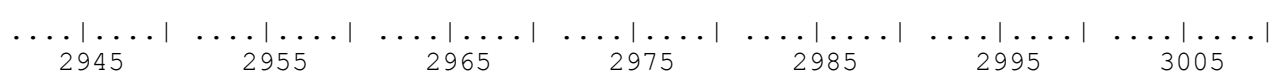

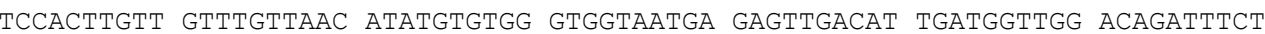
TCCACTTGTT GTTTGTTAAC ATATGTGTGg GTG-TAATGA GAGT-GACAT TGATGGTTGG ACAGATTTCT TCCACATGTT GTTTGTTAAC ATATGTGTGg GTG-TAATGA GAGT-GACAT TGATGGTTGg ACAGATTTCT TCCACTTGTT GTTTGTTAAC ATATGTGTGG GTG-TAATGA GÄT-GACAT TGATGGTTGG ACAGATTTCT

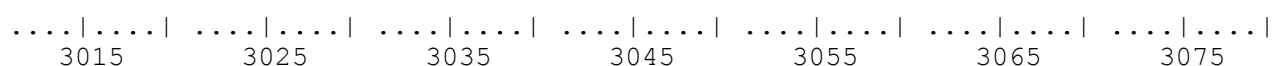
CGTTGGACGA CGATGTTGAT T-GCGTAGTC GTGTTCCATT TTCTGTCTAC ATGTTGATGA AgGAGTGTGT CGTTGGACGA CGATGTTGAT TTGCGTAGTC GTGTTCCATT TTCTGTCTAC ATGTTGATGA AGGA $\overline{\overline{G T G T G T}}$ CGTTGGACGA CGATGTTGAT TTGCGTAGTG GTGTTCCCTT TTCTGTTTAC ATGTTGATTC AGGA $\underline{\overline{G T G T G T}}$ C̈GTTGGACGA CGATGTTGAT TTGCGTAGTC GTGTTCCATT TTCTGTCTAC ÄTGTTGÄGA ÄGGAGTGTGT $\ldots|\ldots| \ldots|\ldots| \ldots|\ldots| \ldots|\ldots| \ldots|\ldots| \ldots|\ldots| \ldots|\ldots| \ldots|\ldots| \ldots|\ldots| \ldots|\ldots|$
3085
3095
3105
3115
3125
3135
3145

-T-TGTGTGT GTGTGTGTGT GTGAATGTGg GATATAGTGT GTTGGGTTGA TTATG--TGT TGATTGTTTT -T-TGTGTGT GTGTGTGTGT GTGCATGTGG GATATAGTGT GTTGGGTTGA TTATG--TGT TGATTGTTTT GTGTGTGTGT GTGTGTGTGT GTGAATGTGG GATCTAGTGT GTTGGGTTGA TTATGCATGT TGATTGTTTT $\ldots \ldots \ldots \ldots \ldots \ldots \ldots \ldots \ldots \ldots \ldots \ldots \ldots \ldots \ldots \ldots$ TGATGTTTT -T-TGTGTGT GTGTGTGTGT GTGAATGTGG GATATAGTGT GTTGGGTTGA TTATG--TGT TGATTGTTTT

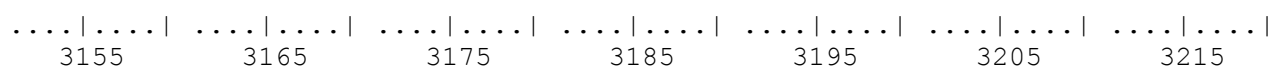

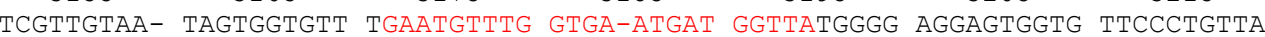

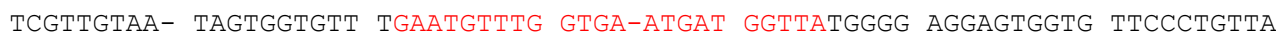

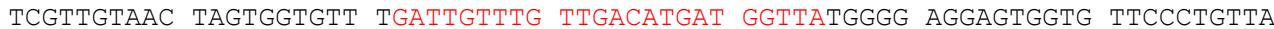

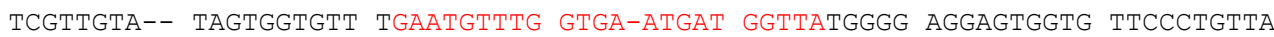

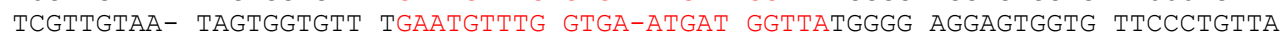

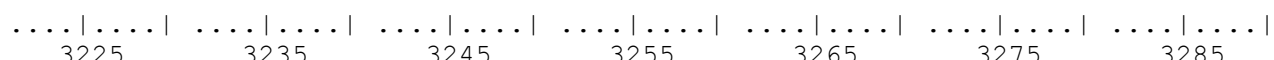
TGTTTCCGTT GTTCAGGTTG TGT------ --GCGTTGTG --------A TGTTCTAGTG TGTGTAATGC TGTTTCCGTT GTTCAGGTTG TGTGTGTTGT GTGTGTTGTG $-\cdots-----$ A TGTTCTAGTG TGTGTAAT GC

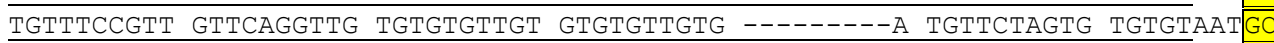

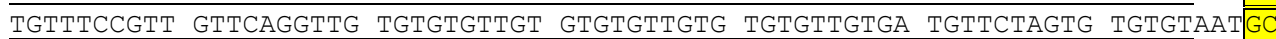
TGTTTCCGTT GTTCAGGTTG TGTGTGTTGT GTGTGTTGTG -------A TGTTCTAGTG TGTGTAAT GC

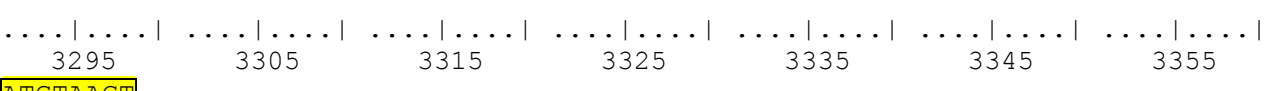

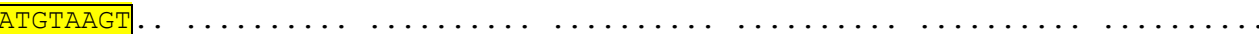

ATGTAAGTA C TCTCAACCAG TCCACTTGTT GTTTGTtAAC ATATGTGTGg GTGtAATGAg AgtGACATTG ATGTAAGTA ATGTAAGTA C TCTCAACCAG TCCACTTGTT GTTTGTTAAC ATATGTGTGg GTGTAATGAg AGTGACATTG

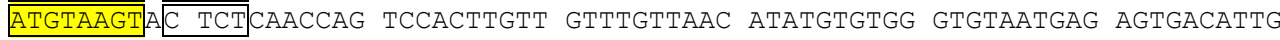
$\ldots|\ldots| \ldots|\ldots| \ldots|\ldots| \ldots|\ldots| \ldots|\ldots| \ldots|\ldots| \ldots|\ldots| \ldots|\ldots| \ldots|\ldots| \ldots \mid$
3365
3375
3385
3395
3405
3415
3425

ATGGT ATGGTTGGAC AgATTTCTCG TTGGACGACG ATGTTGATTT GCGTAGTCGT GTTCCATTTT CTGTCTACAT ATGGTTGGAC AGATTTCTCG TTGGACGACG ATGTTGATTT GCGTAGTCGT GTTCCATTTT CTGTCTACAT

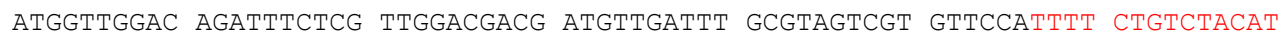


SmBr 18 scaff000011 EST Contig scaff000068 Contig-0

SmBr 18 scaff000011 EST Contig scaff000068 Contig-0

SmBr 18 scaff000011 EST Contig scaff000068 Contig-0

SmBr18 scaff000011 EST Contig scaff000068 Contig-0

SmBr 18 EST Contig scaff000068 Contig-0

SmBr18 scaff000011 EST Contig scaff000068 Contig-0

SmBr 18 scaff000011 EST Contig scaff000068 Contig-0

SmBr18 scaff000011 EST Contig scaff000068 Contig-0

$\mathrm{SmBr} 18$ scaff000011 EST Contig Contig-0 scaff000011 scaff000068

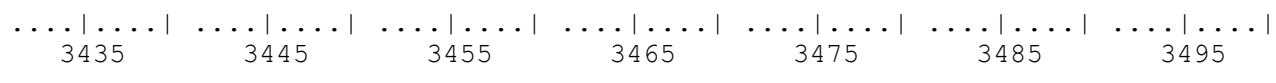

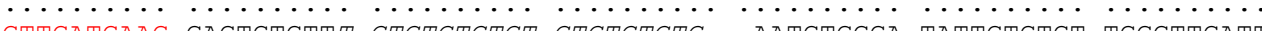
GTTGATGAAG GAGTGTGTTT GTGTGTGTGT GTGTGTGTG- -AATGTGGGA TATTGTGTGT TGGGTTGATT GTTGATGAAG GAGTGTGTTT GTGTGTGTGT GTGTGT--G- -AATGTGGGA TATAGTGTGT TGGGTTGATT GTTGATGAAG GAGTGTGTTT GTGTGTGTGT GTGTGT--GT GAATGTGGGA TATAGTGTGT TGGGTTGATT GTTGATGAAG GAGTGTGTTT GTGTGTGTGT GTGTGT--G- -AATGTGGGA TATAGTGTGT TGGGTTGATT

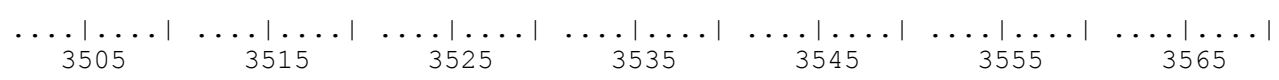

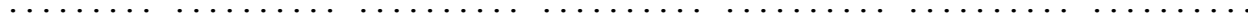

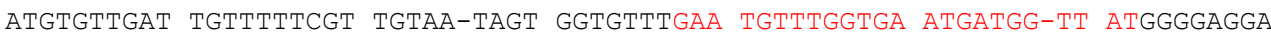

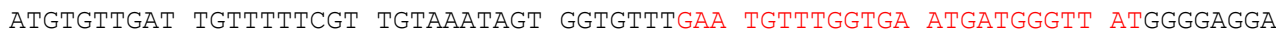

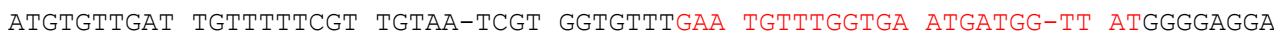

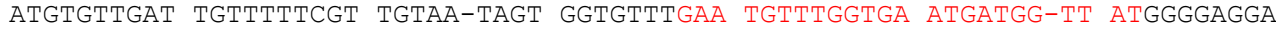

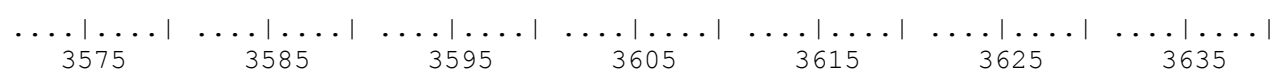

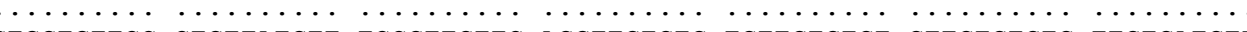
GTGGTGTTCC CTGTTATGTT TCCGTTGTTC AGGTTGTGTG TGTTGTGTGT GTTGTGTGTG TTGTGATGTT

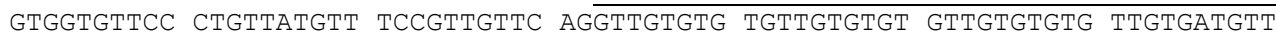

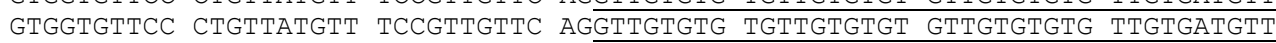

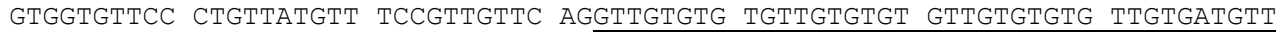

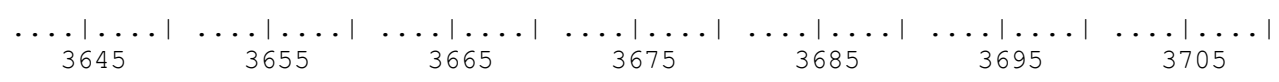

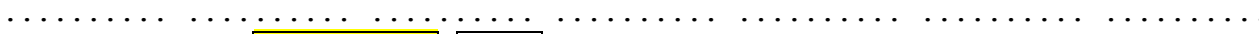
CTAGTGTGTG TAAT GCATGT AAGTA CTCTC AACCAGTCCA ATTGTTGTTT GTTAACATAT GTGTGGGTGT CTAGTGTGTG TAAT GCATGT AAGTA CTCTC AACCAGTCCA CTTGTTGTTT GTTAACATAT GTGTGGGTGT CTAGTGTGTG TAAT GCATGT AAGTA CTCTC AACCAGTCCA CTTGTTGTTT GTTAACATAT GTGTGGGTGT CTAGTGTGTG TAAT GCATGT AAGTA CTCTC AACCAGTCCA CTTGTTGTTT GTTAACATAT GTGTGGGTGT

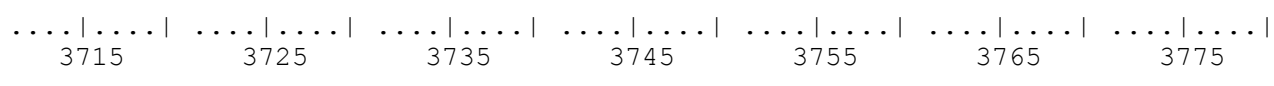
. AATGAGAGTG ACATTGATGG TTGGACAGAT TTCTCGTTGG ACGACGATGT TGATTTGCGT AGTCGTGTTC

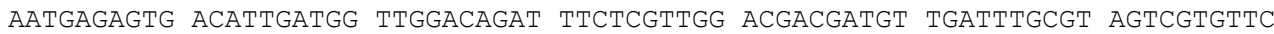

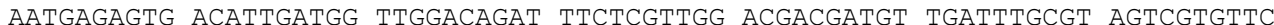

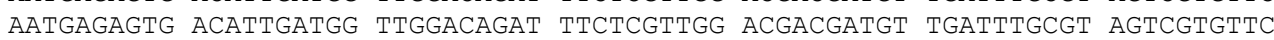

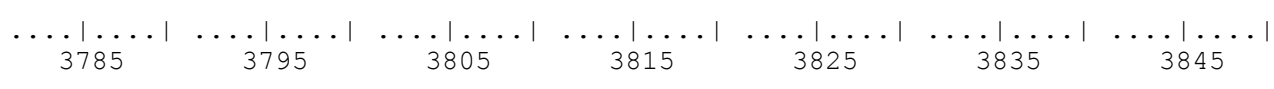

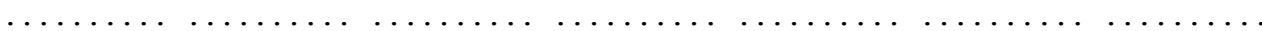

CATTTTCTGT CTACATGTTG ATGAAGGAGT GTGT--GTGT GTGTGTGTG- - -AATGTGG GATATAGTGT CATTTTCTGT CTACATGTTG ATGAAGGA GT GTGTTTGTGT GTGTGTGTGT GTGAATGTGG GATATAGTGT CATTTTCTGT CTACATGTTG ATGAAGGA GT GTGTTTGTGT GTGTGTGTGT G--AATGTGG GATATAGTGT

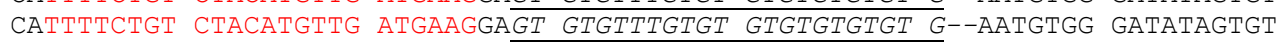

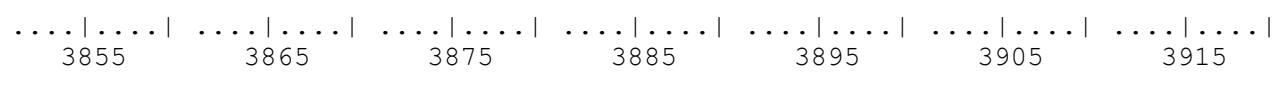
. . . . . . . . . . . . .

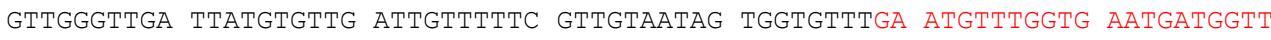

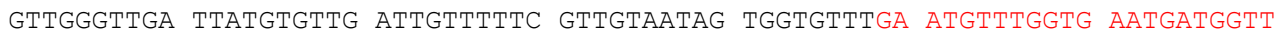

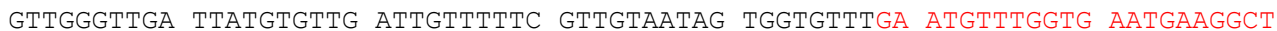

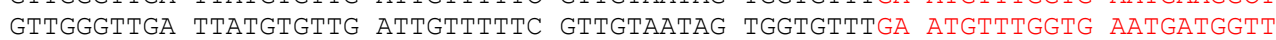

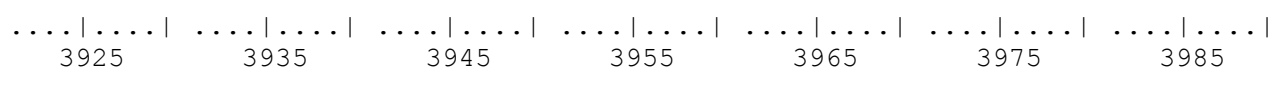
$\ldots \ldots \ldots \ldots \ldots \ldots \ldots \ldots \ldots \ldots \ldots \ldots \ldots$ ATGGGGAGGA GTGGTGTTCC CTGT-TAT-G TTTCCG-TTG TTCAGGTTGT GTGTGTTGTG TGTGTTGTGA ATGgGgAgGA GTGGTGTTCC CTGT-TAT-G TTTCCG-TTG TTCA $\overline{\text { GGTTGT GTGTGTTGTG TGTGTTGTGA }}$ GTGGgTTTCT TTG-T-TTTC TTGTATTtAg TT-CTGATT- TT-A--TT-T CTGT-TTGTC TTT-TTTTTA

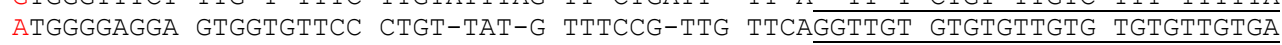

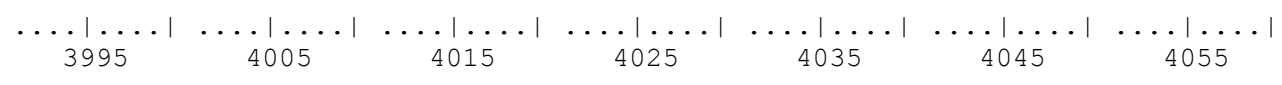

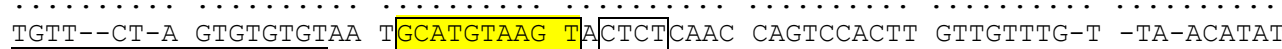
TGTT--CT-A GTGTGTGTAA T GCATGTAAG TA CTCTCAAC CAGTCCACTT GTTGTTTG-T -TA-ACATAT TGTTTGCGGA GTGT-T-T-- T-C-T-T--- TT-T-T---- --GT--A-TT TTTGTTTGGT CTCTA-ATAT TGTT--CT-A GTGTGTGTAA T GCATGTAAG TA CTCTCAAC CAGTCCACTT GTTGTTTG-T -TA-ACATAT $\ldots|\ldots| \ldots|\ldots| \ldots|\ldots| \ldots|\ldots| \ldots|\ldots| \ldots|\ldots| \ldots|\ldots| \ldots|\ldots| \ldots|\ldots|$

$\begin{array}{ccccccc}4065 & 4075 & 4085 & 4095 & 4105 & 4115 & \cdots 125\end{array}$ 
scaff000011 EST Contig scaff000068 Contig-0

$\operatorname{SmBr} 18$ scaff000011 EST Contig scaff000068 Contig-0

$\operatorname{SmBr} 18$ scaff000011 EST Contig scaff000068 Contig-0

SmBr 18 scaff000011 EST Contig scaff000068 Contig-0

SmBr 18 scaff000011 EST Contig scaff000068 Contig-0

SmBr18 scaff000011 EST Contig scaff000068 Contig-0

SmBr 18 scaff000011 EST Contig scaff000068 Contig-0

SmBr 18 scaff000011 EST Contig scaff000068 Contig-0

SmBr18 scaff000011 EST Contig scaff000068 Contig-0

SmBr18 scaff000011 EST Contig scaff000068 Contig-0

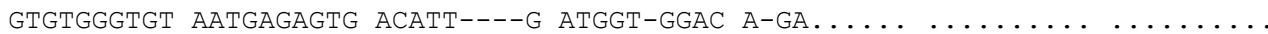
GTGTGGGTGT AATGAGAGTG ACATT---CG CTGGTTGGAC T-GATT-TCT CGTTGTTCGA GGTTGTGTGA -TGTTA-T-T AATTT-ATTT TCTTTTTTCG -TG-TTTTAT TTG-TTCTCT C-TT-TT--A GGTTGA-TGA GTGTGGGTGT AATGAGAGTG ACATT---CG ATGGTTGGAC T-GATTCTCT CGTTGTTCGA GGTTGAGTGA

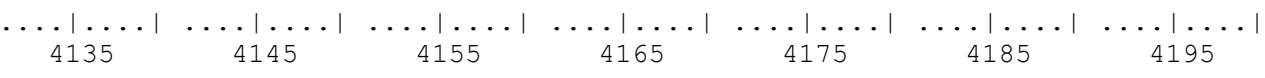

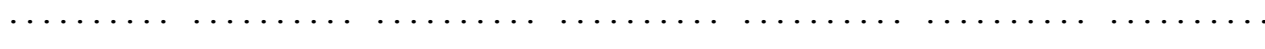

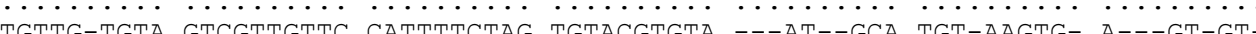
TGAAGAT-TA --CATT---C C-TT--CTCG T-T-C-TG-A GCCATCTG-A TGTGAAGTAC AATCGTCGTC TGAAGATGTA GTCATTGTTC CATTTTCTAg TGTACGTGTA GCCATCTGCA TGTGAAGTAC AATCGTCGTC

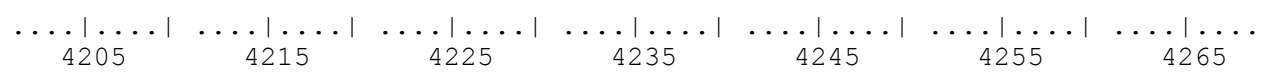

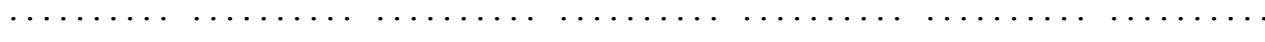

GTTTG-TGTG TGTGTGTGTG TGAATGTGGG ÄAT-TGTGT GTTGGGTTGA T-TATGTGT- T-G-A-TTGT GTTTGCT-TA T-TCT-TGC- TCAA-GTGG- A-ACGTGTAT GTT---TTTT TCTAT-TGTA TAGTACTTGT

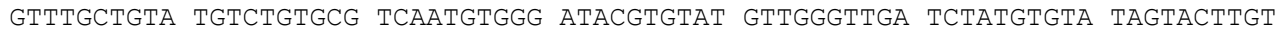

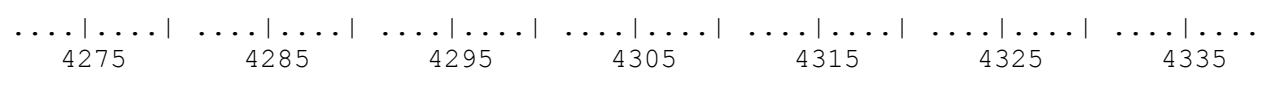

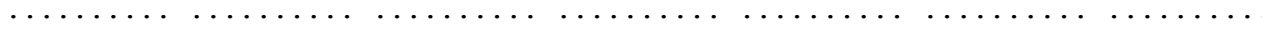

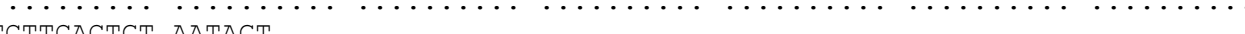
TAT--A-TAT A-TA-TTTTT TATCGTGTTt GTGTCGTAGA TCGTTTTTCA TTTGTTGTAA CTTTGTAGGT TATTCACTAT AATAGTTTTT TATCGTGTTT GTGTCGTAGA TCGTTTTTCA TTTGTTGTAA CTTTGTAGGT

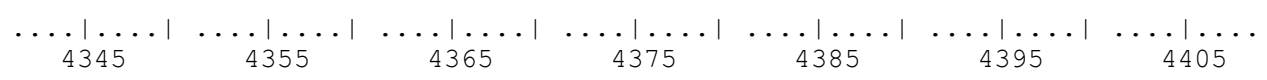

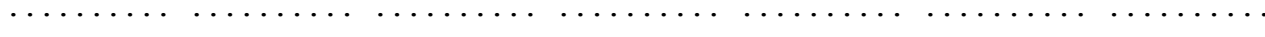

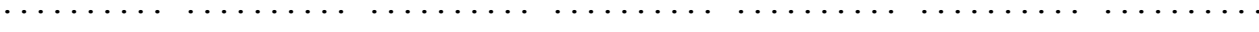

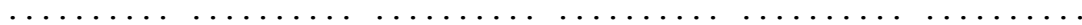
CAAAAATGAT CCTCTCTCCG TCGTTTTATT CGTTGCTTTG TTAATTACAC TTTTGACTTT TCTTTTACGA CAAAAATGAT CCTCTCTCCG TCGTTTTATT CGTTGCTTTG TTAATTACAC TTTTGACTTT TCTTTTACGA

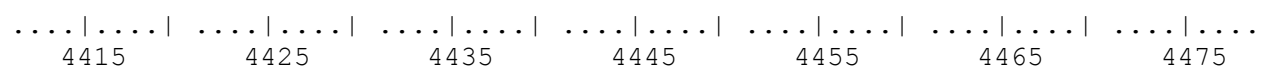

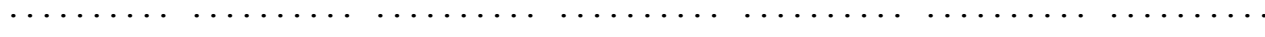

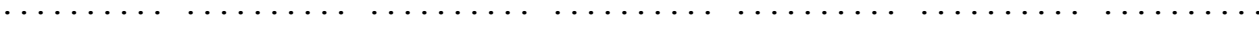

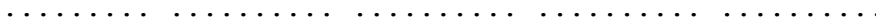
TTGTTTCTAT TTTTTTGACA GATAATTCCC TTTTAAACAT TCGAAATTAA AAATACGAAG TTGAGCGAAG TTGTTTCTAT TTTTTTGACA GATAATTCCC TTTTAAACAT TCGAAATTAA AAATACGAAG TTGAgCGAAG

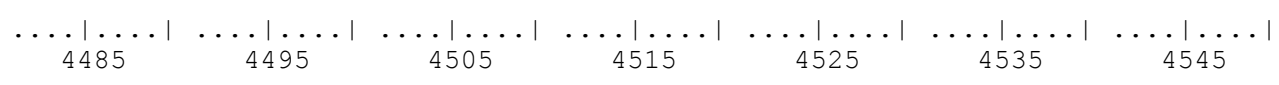

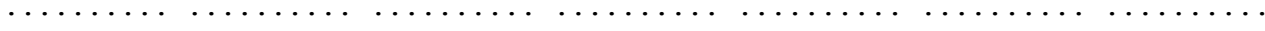

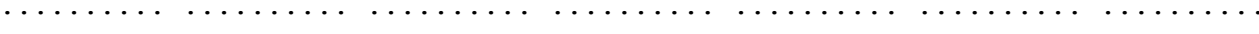
AgATCTCATt TCAACGAATt TTtTATCAAG CTGTACAATC GTACTAATAT GGAAAATtGT TTTTtTGtTA AgATCTCATT TCAACGAATT TTTTATCAAg CTGTACAATC GTACTAATAT GGAAAATTGT TTTTTTGTTA

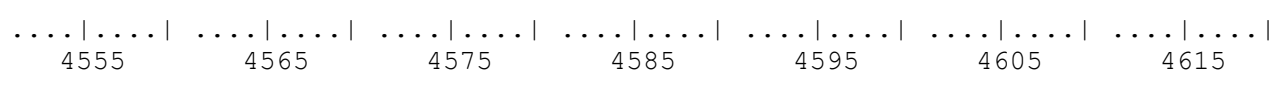

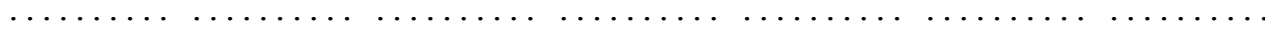

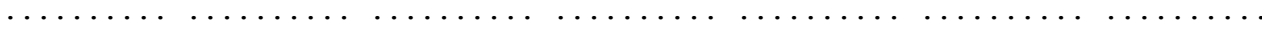
$\begin{array}{lllll}\ldots \ldots \ldots & \ldots \ldots \ldots & \ldots \ldots \ldots & \ldots \ldots \ldots & \ldots \ldots \ldots\end{array}$ AAGTACTAAT GGGAACACAA ATAACCGAGA TGATGGAAAA TAATCTTTCT ATTGAATCAT CTTGGTTATT AAgTACTAAT GGGAACACAA ATAACCGAGA TGATGGAAAA TAATCTTTCT ATTGAAtCAT CTTGGTTATt

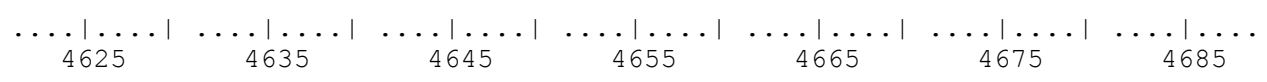

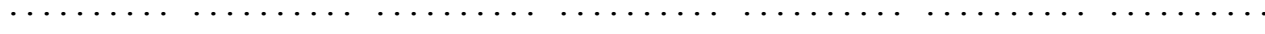

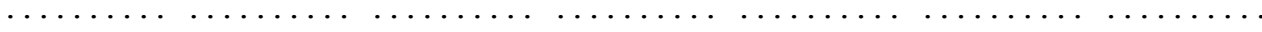
CGTGTGCACT TTAAGGGCTG AATTTATAAC AGAAGTTTGC TGAAACGGCC TCAAATCATT TAGTTTTTCC

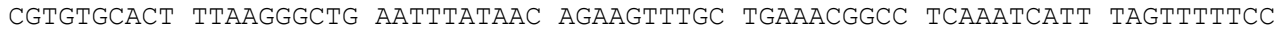

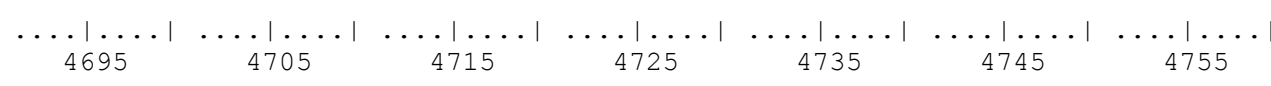

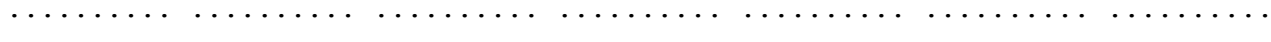

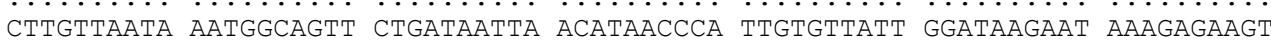

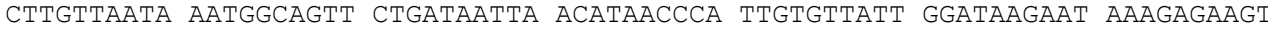


SmBr 18 scaff000011 EST Contig scaff000068 Contig-0

SmBr 18 scaff000011 EST Contig scaff000068 Contig-0

SmBr 18 scaff000011 EST Contig scaff000068 Contig-0

SmBr 18 scaff000011 EST Contig scaff000068 Contig-0

SmBr 18 scaff000011 EST Contig scaff000068 Contig-0

SmBr18 scaff000011 EST Contig scaff000068 Contig-0

SmBr18 scaff000011 EST Contig scaff000068 Contig-0

SmBr18 scaff000011 EST Contig scaff000068 Contig-0

SmBr 18 scaff000011 EST Contig scaff000068 Contig-0

SmBr 18 scaff000011

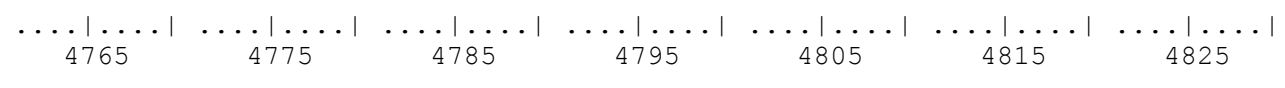

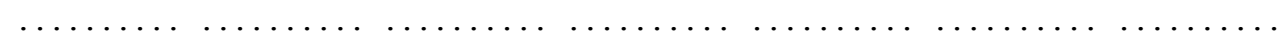

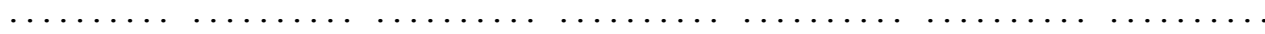

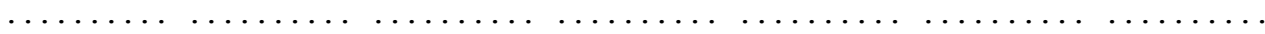
CAGCGAAGAA AATTTTCTTT TCCACTAAAT CGTTTACTTA AGCTGTACAT TCTTGTTTAT AATCATACGG CAgCGAAGAA AATTTTCTTT TCCACTAAAT CGTTTACTTA AgCTGTACAT TCTTGTTTAT AATCATACGG

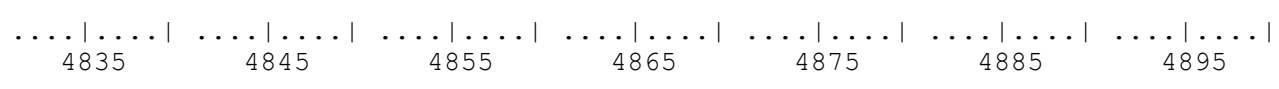

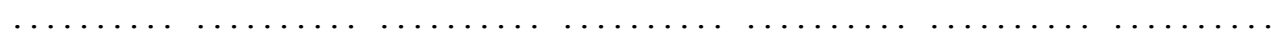

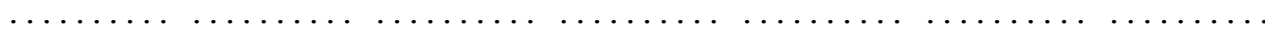

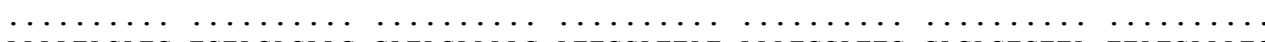
AAAATACATG TCTAGAgAAg GATAgAAAAg ATTGCATTAT AAATGGATTC GAGACTCTTA TTATCAAATG

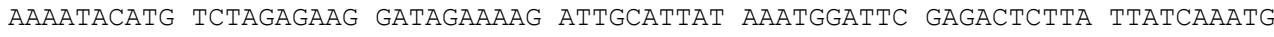

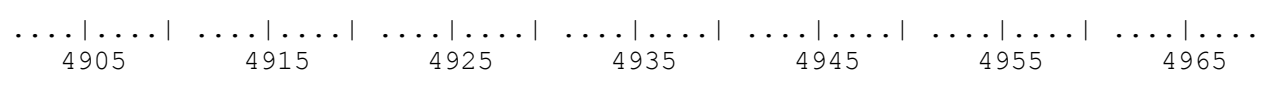

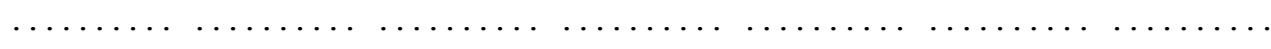

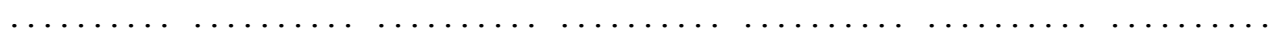

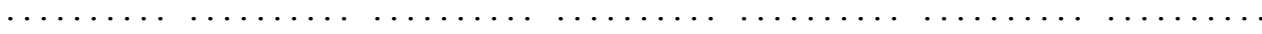

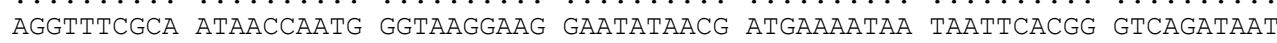
AgGtTtCGCA ATAACCAAtg GgtAAgGAAg GAAtAtAACG ATGAAAATAA TAATtCACGg gTCAgAtAAT

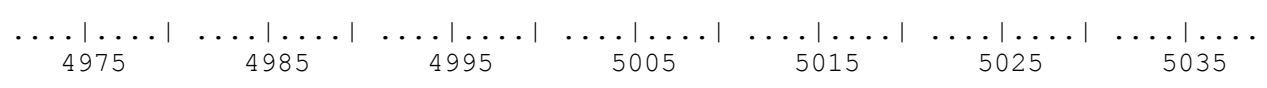

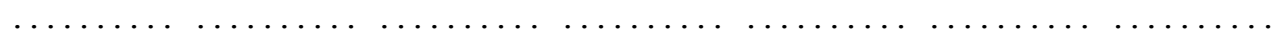
TGTCCAATTG ACAGGTATGA AGAAAAATGA CAAACATGA GGTCATAATA AAAACTGAAT AATAATCATG tgtccAAttg ACAgGtAtgA AgAAAAAtga cAAACAtgAa ggtcAtAAtA AAAACtgaAt AAtAAtcAtg

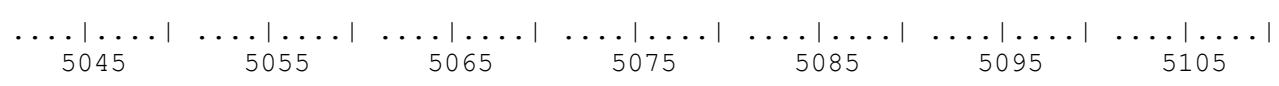

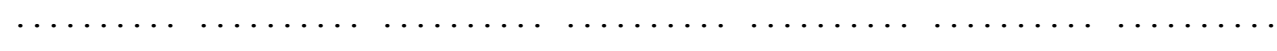

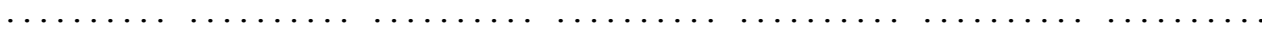
AAAACTTGTA ATAATAATAA TAATAATAAC TGATTAGAGT TTAAAATTAC AAAACAGTTA TCTAAAAAAT

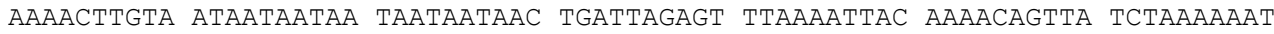

$\ldots|\ldots| \ldots|\ldots| \ldots|\ldots| \ldots|\ldots| \ldots|\ldots| \ldots|\ldots| \ldots|\ldots| \ldots|\ldots| \ldots|\ldots| \ldots \mid$
5115
5125
5135
5145
5155
5165
5175

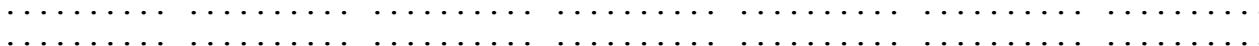

CÄTÄAтÄ CAATAATAAC CGAAGAAAAA CGAAGAAAAA AGGGAGAAAA ACCGAAACAT ACTACTAAAG AGAATCAGGG

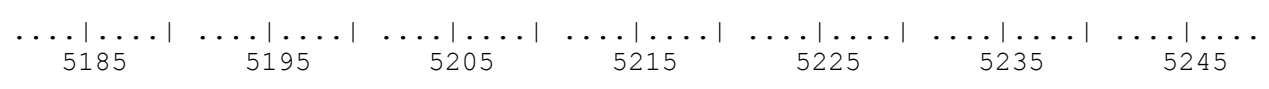

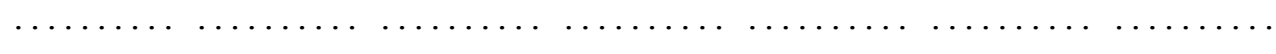

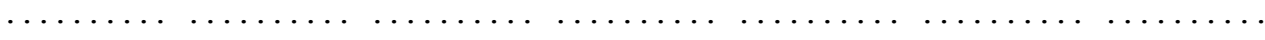
GCAAGGAAGG ATGAGAGGGG TTGGACCAGT

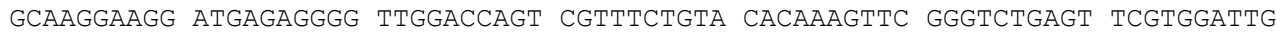

\begin{tabular}{|c|c|c|c|c|c|c|}
\hline$\cdots\left|{ }_{5255}{ }^{\prime}\right|$ & $\cdots\left|{ }_{5265} \cdots\right|$ & $\cdots\left|{ }_{5275}{ }^{\prime}\right|$ & $\cdots\left|{ }_{5285} \cdots\right|$ & $\cdots{ }_{5295}{ }^{\prime}$ & $\cdots \mid{ }_{5305}{ }^{\prime}$ & $\cdots \mid \cdots$ \\
\hline$\cdots$ & $\cdots$ & $\cdots \cdots$ & $\cdots \cdots$ & $\cdots$ & $\cdots$ & \\
\hline & & & & & & \\
\hline & $\cdots \cdots$ & $\cdots$ & $\cdots$ & $\cdots$ & $\cdots$ & \\
\hline $\mathrm{CC}$ & GGCAATGCAT & AAAI & $\mathrm{TTC}$ & CTTCGA & $\mathrm{ATTC}$ & $\mathrm{CTC}$ \\
\hline TCC & GGCAATGCAT & AAAATATTGA & TTCAATTTCC & CTTCGATCCA & ATTCATTTGA & $\mathrm{CTG}$ \\
\hline
\end{tabular}

\begin{tabular}{|c|c|c|c|c|c|c|}
\hline$\cdots \mid{ }_{5325}$ & 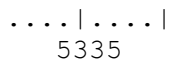 & $\begin{array}{c}\cdots|\ldots| \\
5345\end{array}$ & $\begin{array}{c}\cdots|\ldots| \\
5355\end{array}$ & $\cdots+1 \ldots 1$ & $\cdots+1 \ldots 1$ & $\begin{array}{c}\cdots \\
5385\end{array}$ \\
\hline$\cdots$ & $\cdots$ & $\cdots$ & $\cdots \cdots$ & $\cdots$ & $\cdots$ & • \\
\hline - & - & $\cdots$ & & & & \\
\hline & $\cdots$ & $\ldots .$. & & $\cdots$ & & \\
\hline . & GAA & TTGCAC & GTG & TTA & $\mathrm{GCT}$ & $\mathrm{AG}$ \\
\hline $\mathrm{AC}$ & GAAAACTCTT & TTGCAGTGAT & GTGTTCACAG & TTAATTAAАT & GCTCCTGTAT & AGT \\
\hline
\end{tabular}

\begin{tabular}{|c|c|c|c|c|c|c|}
\hline$\cdots \mid{ }_{5395}$ & $\cdots+\cdots \mid$ & $\cdots+{ }_{5415}$ & 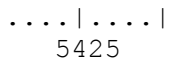 & $\cdots+{ }_{5435}$ & $\cdots+\cdots \mid$ & 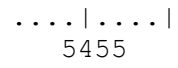 \\
\hline$\ldots \ldots \ldots$ & $\ldots \ldots \ldots$ & $\ldots \ldots \ldots$ & $\cdots \cdots \cdots$ & $\ldots \ldots \ldots$ & $\cdots \cdots \cdots$ & $\cdot$ \\
\hline . & $\cdots \cdots$ & $\cdots \cdots$ & $\cdots \cdots$ & $\cdots \cdots$ & . & \\
\hline
\end{tabular}


EST Contig scaff000068 Contig-0

SmBr18 scaff000011 EST Contig scaff000068 Contig-0

SmBr 18 scaff000011 EST Contig scaff000068 Contig-0

SmBr18 scaff000011 EST Contig scaff000068 Contig-0

$\mathrm{SmBr} 18$ scaff000011 EST Contig scaff000068 Contig-0

SmBr 18 scaff000011 EST Contig scaff000068 Contig-0

$\mathrm{SmBr} 18$ scaff000011 EST Contig scaff000068 Contig-0

SmBr 18 scaff000011 EST Contig scaff000068 Contig-0

SmBr 18 scaff000011 EST Contig scaff000068 Contig-0

$\operatorname{SmBr} 18$ scaff000011 EST Contig scaff000068 Contig-0

ATTGTTTTAC ATTCTCCTTT TAAAAGCCAT GTCGGATAGT GTTCTGAGAT GCGTTTTGGA AAGTGCTCGC ATTGTTTTAC ATTCTCCTTT TAAAAGCCAT GTCGgAtAgt GTTCTGAGAT GCGTTTTGgA AAgTGCTCGC

\begin{tabular}{|c|c|c|c|c|c|c|}
\hline$\cdots \mid{ }_{5465}$ & $\cdots+1 \ldots$ & $\cdots \mid{ }_{5485}$ & $\cdots|\cdots|$ & $\cdots+1 \ldots$ & $\cdots||_{5515}$ & $\cdots \mid{ }_{5525}$ \\
\hline$\cdots \cdots \cdots$ & $\cdots \cdots$ & $\cdots \cdots$ & $\cdots \cdots$ & $\cdots$ & $\cdots$ & \\
\hline$\ldots \ldots$ & $\ldots \ldots$ & $\cdots \cdots$ & $\ldots \ldots \ldots$ & $\cdots \cdots$ & $\cdots \cdots$ & \\
\hline$\ddot{G C G G C}$ & $\ddot{\text { CAATGTAGCC }}$ & $\ddot{C A C A C}$ & $\ddot{A} \dot{A G T A}$ & ATTTATAGAT & $\ddot{A C A C G T T G A T}$ & \\
\hline
\end{tabular}
tTtgtgCGgC CAATGtAgCC TGCCCCACAC tAgCAAgtAA ATtTAtAgAt ACACGTtGAt GTGgCCCAAA

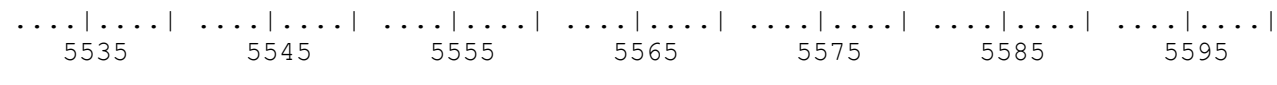

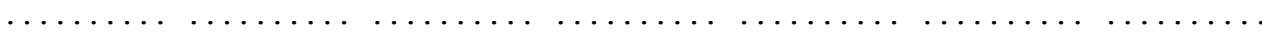

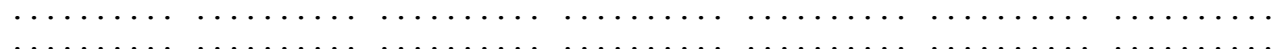
GAgGAAgtTT ATACTTAACA CATCGgAgAA tgGtgGgCCG GCTAGAgAAA ACAATTCTGA GCTTAGCTGA GAgGAAgTTT ATACTTAACA CATCGgAgAA tgGtgGgCCG GCtAgAgAAA ACAATTCTGA GCTTAgCTGA

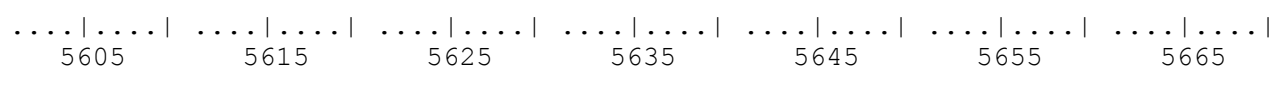

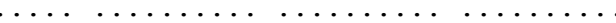

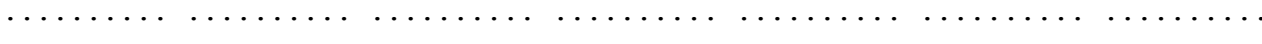

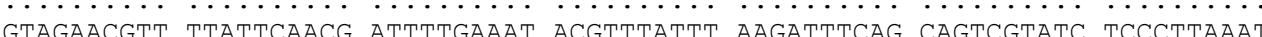
GTAGAACGTT TTATTCAACG ATTTTGAAAT ACGTTTATTT AAGATTTCAG CAGTCGTATC TCCCTTAAAT

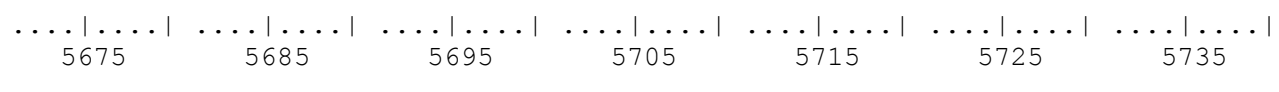
$\ldots \ldots \ldots$

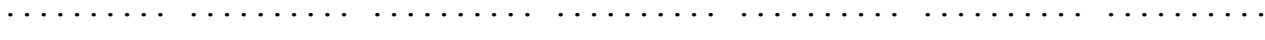

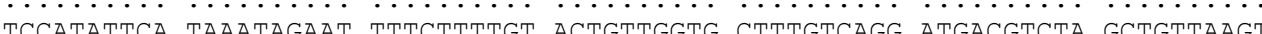
TCCATATTCA TAAATAGAAT TTTCTTTTGT ACTGTTGgTG CTTTGTCAGG ATGACGTCTA GCTGTTAAGT

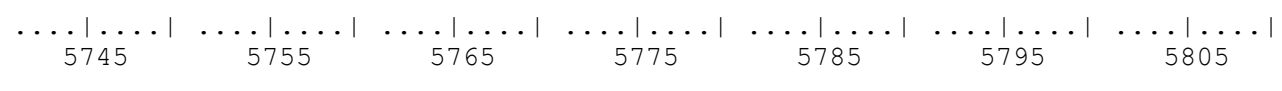

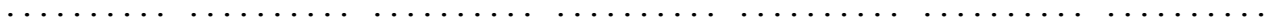
$\ldots \ldots \ldots \cdots \cdots \cdots \cdots \cdots \cdots \cdots \cdots \cdots \cdots \cdots \cdots$ TGCTACCGAC GAATCTAGGT GGATAACCAT TCCCAAAGAG AATCTGTCGA CGTCGAAGTA GTTCCTCATC TGCTACCGAC GAATCTAGGT GGATAACCAT TCCCAAAgAG AATCTGTCGA CGTCGAAGTA GTTCCTCATC

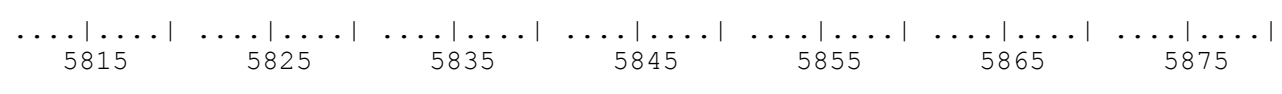

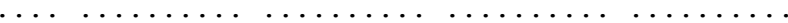
$\cdots \cdots \cdots \cdots \cdots \cdots \cdots \cdots \cdots \cdots \cdots \cdots \cdots \cdots \cdots \cdots \cdots \cdots \cdots \cdots$ TATTGTGTCA GTAGgACATA CTCGCCTGAT CCTTGAgGAT AAAGAGTGAA TCAAGTTCCT CTTTCTACTC TATTGTGTCA GTAGgACATA CTCGCCTGAT CCTTGAgGAT AAAgAgTGAA TCAAGTTCCT CTTTCTACTC

\begin{tabular}{|c|c|c|c|c|c|c|}
\hline$\cdots\left|{ }_{5885} \cdots\right|$ & $\cdots \mid{ }_{5895}{ }^{\prime}$ & $\cdots \mid{ }_{5905}$ & 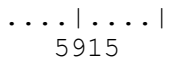 & 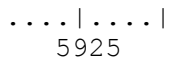 & $\cdots+1 \ldots 1$ & $\cdots+\cdots$ \\
\hline$\ldots \ldots$ & $\cdots$ & $\cdots$ & $\cdots$ & • & $\cdot$ & \\
\hline$\cdots$ & $\ldots \ldots \ldots$ & $\cdots \ldots \ldots$ & $\ldots \ldots \ldots$ & $\ldots \ldots \ldots$ & $\cdots \cdots$ & \\
\hline$\ddot{\mathrm{CCC}}$ & $\ddot{\dot{G G A}}$ & $\begin{array}{l}\cdots \\
\text { ITTAC }\end{array}$ & $\ddot{\text { TGCCCATTCC }}$ & $\ddot{\text { CGCTTT }}$ & $\begin{array}{c}\cdots \\
\text { CATC }\end{array}$ & \\
\hline
\end{tabular}
AGTGGTACCC GGCTATGGAA ATTCGTTtAC TGCCCATtCC AGGTCGCTTT TCTATACATC AAAAAAAAGA

\begin{tabular}{|c|c|c|c|c|c|c|}
\hline 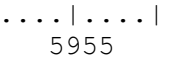 & $\begin{array}{c}\ldots|\ldots| \\
5965\end{array}$ & $\begin{array}{c}\ldots|\ldots| \ldots \mid \\
5975\end{array}$ & ${ }_{5985}^{\ldots \mid}$ & ${ }_{599}^{\ldots|\ldots|}$ & ${ }_{6005}^{\ldots \mid}$ & ${ }_{6015}$ \\
\hline$\cdots \cdots$ & $\cdots$ & $\cdots \cdots \cdots$ & $\cdots \cdots$ & $\cdots$ & . & \\
\hline$\cdots$ & $\cdots$ & $\cdots$ & $\cdots \cdots \cdots$ & $\cdots \cdots$ & $\cdots$ & \\
\hline $\begin{array}{c}\cdots \\
T G T\end{array}$ & $\ddot{\text { TCTGC }}$ & $\ddot{G T G}$ & $\ddot{A T A G}$ & $\ddot{\cdots A}$ & $\dot{\cdots A}$ & \\
\hline
\end{tabular}

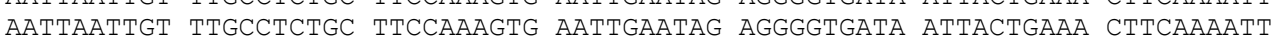

\begin{tabular}{|c|c|c|c|c|c|c|}
\hline$\cdots \mid{ }_{6025}{ }^{\prime}$ & $\cdots{ }_{6035}{ }^{\prime}$ & $\cdots{ }_{6045}$ & $\cdots\left|{ }_{6055} \cdots\right|$ & $\cdots{ }_{6065}{ }^{\prime}\left|{ }^{\prime}\right|$ & $\cdots\left|{ }_{6075} \cdots\right|$ & $\cdots \mid{ }_{6085} \cdots 1$ \\
\hline$\cdots \cdots$ & $\cdots$ & $\cdots$ & $\cdots$ & $\cdots$ & $\cdots$ & \\
\hline$\cdots$ & $\cdots \cdots$ & $\cdots \cdots$ & $\cdots \cdots$ & - & - & \\
\hline & . & .. & $\cdots$ & $\cdots$ & $\cdots$ & \\
\hline TCATTGAGGT & TTATATСTTC & TTCACAAATA & ATGAAAGTAT & CATCCATAGA & TCACCTATAT & AAGTGA \\
\hline TCATTGAGGT & TTATATСTTC & TTCACAAATA & ATGAAAGTAT & CATCCATAGA & TCACСТАТАТ & AAGTGAAAT \\
\hline
\end{tabular}


$\mathrm{SmBr} 18$ scaff000011 EST Contig scaff000068 Contig-0

$\mathrm{SmBr} 18$ scaff000011 EST Contig scaff000068 Contig-0

SmBr 18 scaff000011 EST Contig scaff000068 Contig-0

SmBr 18 scaff000011 EST Contig scaff000068 Contig-0

SmBr18 scaff000011 EST Contig scaff000068 Contig-0

SmBr18 scaff000011 EST Contig scaff000068 Contig-0

SmBr18 scaff000011 EST Contig scaff000068 Contig-0

SmBr18 scaff000011 EST Contig scaff000068 Contig-0

SmBr 18 scaff000011 EST Contig scaff000068 Contig-0

$\operatorname{SmBr} 18$ scaff000011

\begin{tabular}{|c|c|c|c|c|c|}
\hline$\cdots \underset{6095}{\cdots \mid}$ & $\cdots \mid{ }_{6105}$ & $\cdots|\cdots|$ & $\cdots|\cdots|$ & $\cdots|\cdots|$ & $\left.\cdots \underset{6145}{\cdots \mid} \cdots\right|_{6155}$ \\
\hline
\end{tabular}

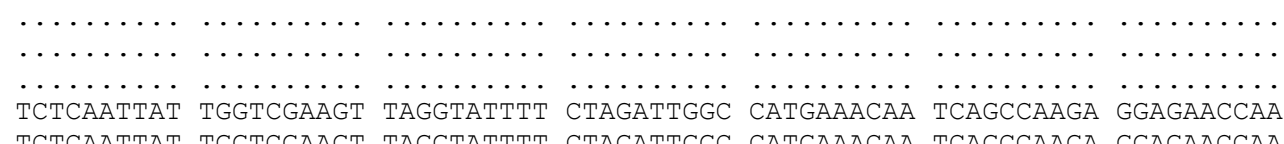
CAAGA GGAGAACCAA

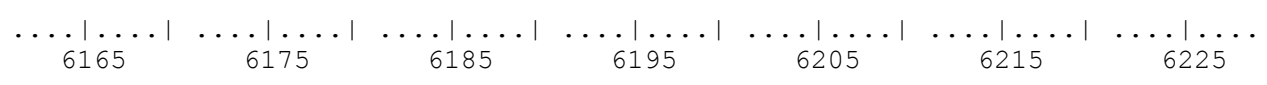

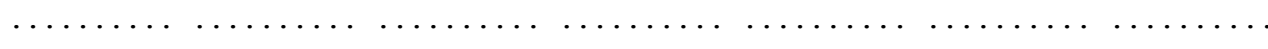

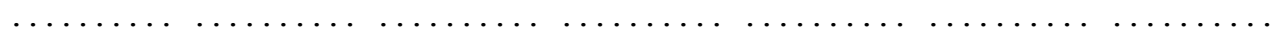

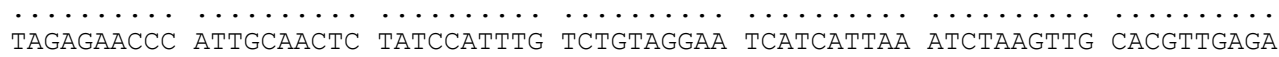

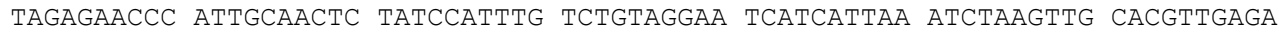

\begin{tabular}{|c|c|c|c|}
\hline$\cdots\left|{ }_{6235} \cdots\right|$ & $\cdots{ }_{6245} \cdots \mid$ & $\cdots\left|{ }_{6255} \cdots\right|$ & $\left.\cdots\right|_{6265} \cdots \mid$ \\
\hline
\end{tabular}

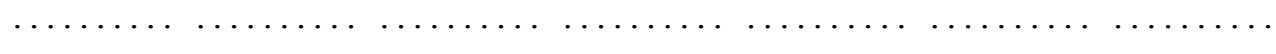

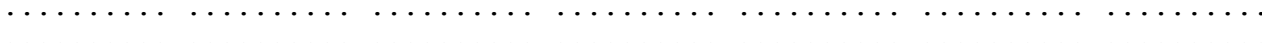
GTACTTCGTA GAAGTAGCTC TTCTAGATAA TGTTTTGGGA TACAAATATC AAGGTTATTG TTTTCAATAT

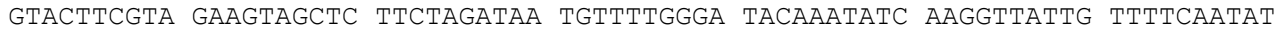

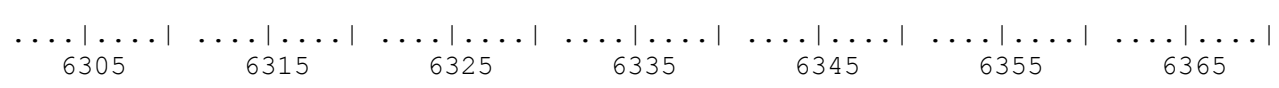

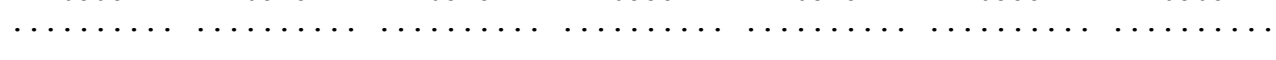

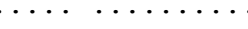
AgtTACATAA ÄÄÄATAGT TTCTGTGÄA

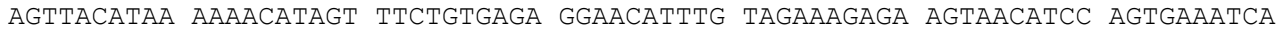

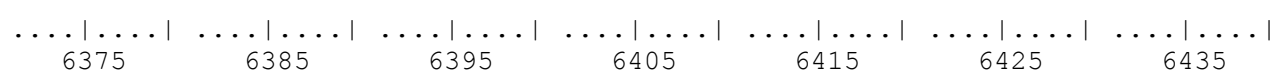

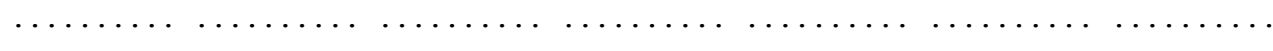

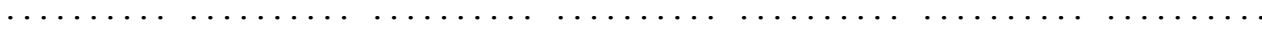

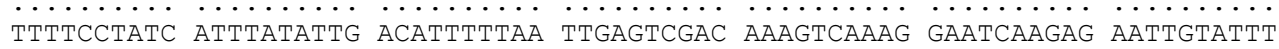

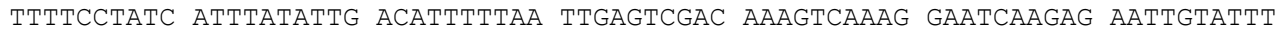

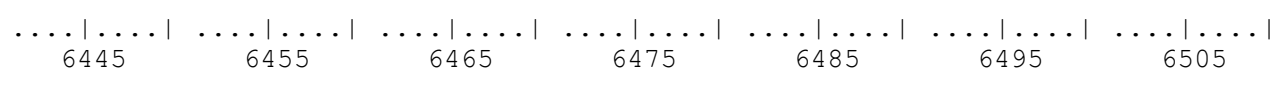

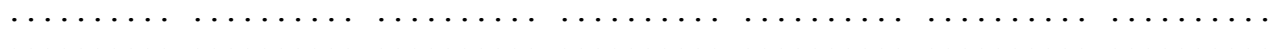

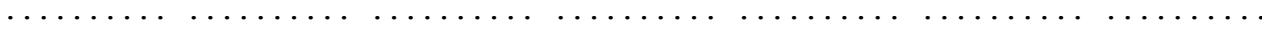
ATTGATATGA TTTTTAACGG ATTGTAAAAT ATCAACTGGC CATTTAGCAA TCAGATGATA CAGAGAGTCG

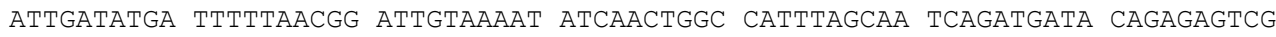

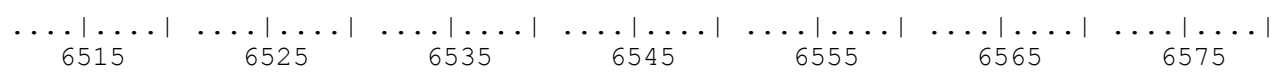

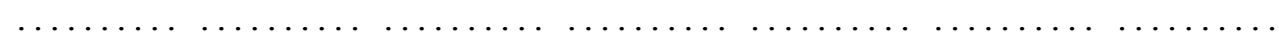

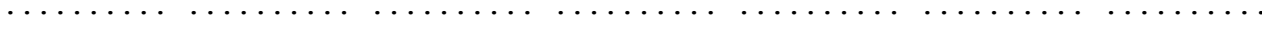
TCCATATCGA GCATAGGATG TAGAGGGACA TTATAACTAT GAACTTTTGT GAGTCCATAT AGTCTTGGTA

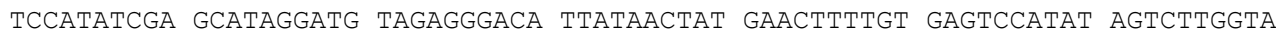

\begin{tabular}{|c|c|c|c|c|c|c|}
\hline$\cdots||_{6585} \cdots \mid$ & $\left.\cdots\right|_{6595} \ldots 1$ & $\left.\cdots\right|_{6605} \cdots \mid$ & 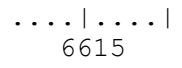 & $\left.\cdots\right|_{6625} \ldots \mid$ & $\left.\cdots\right|_{6635} \ldots \mid$ & $\left.\cdots\right|_{6645} \ldots \mid$ \\
\hline . & & - & & & & \\
\hline$\cdots$ & & & & $\ldots \ldots$ & $\cdots \cdots$ & \\
\hline TÄACGGÄACC & C.......... & ATATGTTCAT & AGAGGTCACA & ÄTMA....... & Ä......... & т.......... \\
\hline AACGGAACC & CGATGGTCTA & ATATGTTCAT & AGAGGTCACA & ATTGATAGTA & AАСТCATCTT & TCATCTTC \\
\hline
\end{tabular}

\begin{tabular}{|c|c|c|c|c|c|c|}
\hline$\cdots{ }_{6655} \ldots \mid$ & $\cdots{ }_{6665} \cdots \mid$ & $\cdots{ }_{6675}{ }^{\prime} \mid$ & $\cdots+\left.\right|_{6685} \ldots \mid$ & 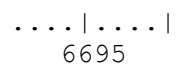 & $\cdots \mid{ }_{6705}$ & ${ }_{6715}^{\ldots|\ldots|}$ \\
\hline . & .. & $\cdots$ & $\ldots$ & $\cdots \cdots$ & $\cdots$ & \\
\hline
\end{tabular}

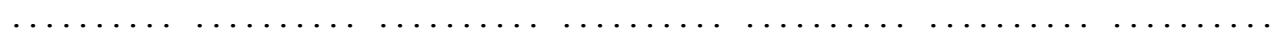
TAGGATTTTA ATAAGCATCT GTTCCGTTTT TTTCAGTTGT CTTTTTGAAC GGTTTCTTTG GAGAATTTGA

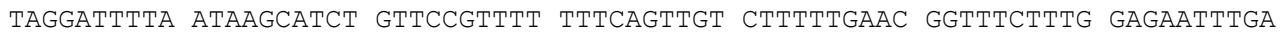

\begin{tabular}{|c|c|c|c|c|c|c|}
\hline$\cdots|\cdots|$ & ${ }_{6735}{ }^{\prime \mid}$ & $\cdots|\cdots|$ & $\cdots|\cdots|$ & $\begin{array}{c}\cdots|\ldots| \\
6765\end{array}$ & $\begin{array}{c}\ldots|\ldots| \\
6775\end{array}$ & $\begin{array}{c}\cdots|\ldots| \\
6785\end{array}$ \\
\hline & $\cdots$ & $\cdot$ & $\cdots$ & . & . & \\
\hline
\end{tabular}


EST Contig scaff000068 Contig-0

SmBr18 scaff000011 EST Contig scaff000068 Contig-0

SmBr 18 scaff000011 EST Contig scaff000068 Contig-0

SmBr18 scaff000011 EST Contig scaff000068 Contig-0

$\mathrm{SmBr} 18$ scaff000011 EST Contig scaff000068 Contig-0

SmBr 18 scaff000011 EST Contig scaff000068 Contig-0

$\mathrm{SmBr} 18$ scaff000011 EST Contig scaff000068 Contig-0

SmBr 18 scaff000011 EST Contig scaff000068 Contig-0

SmBr 18 scaff000011 EST Contig scaff000068 Contig-0

$\operatorname{SmBr} 18$ scaff000011 EST Contig scaff000068 Contig-0

CAGGATCATA TAATATCGAA CACAATTTGG TGACATAGTC AAGACGATCC ACAAAGACGA TTCTCAGACC

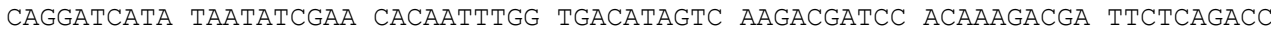

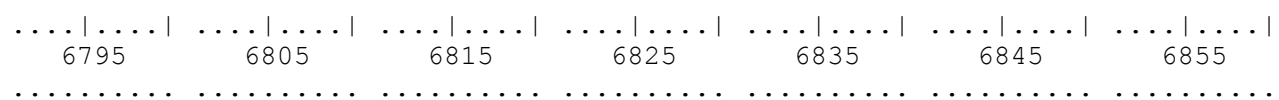

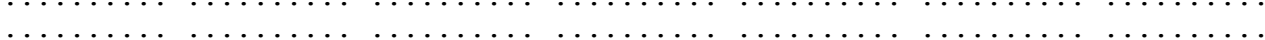
CTTGTCTAGG AGACACAACA CTAAATCCTG ATTTTTCGGA AGATCAGATA ATTGAGATCT ATGCTCTTTT

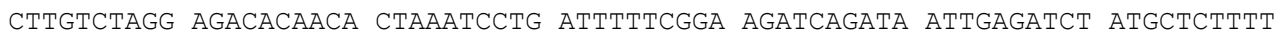

\begin{tabular}{|c|c|c|c|c|c|}
\hline$\cdots\left|{ }_{6865} \cdot \ldots\right|$ & $\cdots{ }_{6875} \cdots \mid$ & $\cdots \mid{ }_{6885} \cdot{ }^{\prime}$ & $\cdots \mid \begin{array}{l}6895 \\
\cdots 1\end{array}$ & $\begin{array}{c}\cdots \\
6905\end{array}$ & $\begin{array}{c}\cdots \\
6915\end{array}$ \\
\hline
\end{tabular}
$\ldots \ldots$

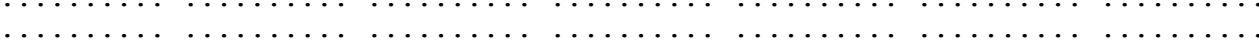
GTGAGTAATC GACTAATTGT AGGTATTTTC TTCACATATT GATAACAACA GTCTACAAGA GTTGTTTTGA GTGAGTAATC GACTAATTGT AgGTATTTTC TTCACATATT GATAACAACA GTCTACAAGA GTTGTTTTGA

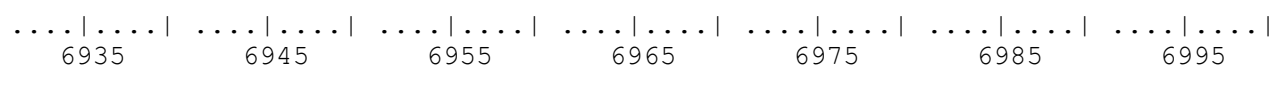

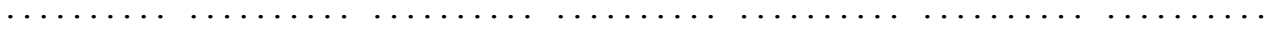
AАTGCTATA AATGCTCATA ATCTTTATTA GAtACTGGAA tAAAgtCACT TGTCTGATGA ATGAgGCTTt CAACCTAGAT

\begin{tabular}{|c|c|c|c|c|c|c|}
\hline$\cdots>1 \ldots 1$ & $\begin{array}{c}\cdots \mid \ldots 1 \\
7015\end{array}$ & $\begin{array}{c}\cdots|\ldots| \\
7025\end{array}$ & $\begin{array}{c}\cdots \\
7035\end{array}$ & 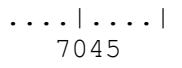 & $\begin{array}{c}\cdots \\
7055\end{array}$ & $\begin{array}{c}\cdots 7065 \\
70\end{array}$ \\
\hline$\cdots$ & $\cdots$ & $\cdots$ & $\cdots$ & $\cdots$ & $\cdots$ & \\
\hline${ }^{\circ}$ & $\cdots$ & $\cdots \cdots$ & $\cdots$ & e & & \\
\hline TMA. & AGTTCATTAA & TGTTAGTTGA & $\ddot{A G T A C T A C A A}$ & ÄATTTAGGAA & CÄАСТTTAAT & CTATÄÄATT \\
\hline ГTAGCGTTG & AGTTCATTAA & TGTTAGTTGA & AGTACTACAA & AATTTAGGAA & CAACATTTAT & $\mathrm{CT}$ \\
\hline
\end{tabular}

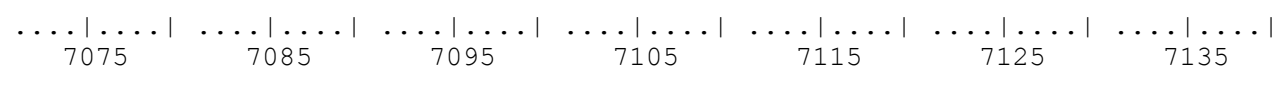

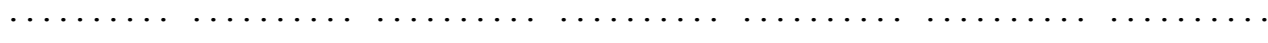

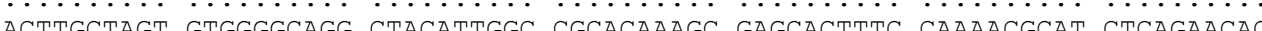
ACTTGCTAGT GTGGgGCAGG CTACATTGgC CGCACAAAGC GAGCACTTTC CAAAACGCAT CTCAGAACAC

\begin{tabular}{|c|c|c|c|c|c|}
\hline$\cdots|\cdots|$ & $\cdots|\cdots|$ & $\cdots|\cdots|$ & $\cdots \mid \ln _{7175}$ & $\cdots \underset{7185}{\cdots \mid}$ & $\cdots{ }_{7195} \cdots|\cdots| \ldots \mid$ \\
\hline
\end{tabular}
$\cdots \cdots \cdots \cdots \cdots \cdots \cdots \cdots \cdots \cdots \cdots \cdots \cdots \cdots \cdots \cdots \cdots \cdots \cdots \cdots \cdots \cdots \cdots \cdots \cdots \cdots \cdots \cdots$ TATCCGACAT GGCTTTTAAA AgGAGAATGT AAAACAATTA CTAGTTCTAT ACAGGAGCAT TTAATTAACT TATCCGACAT GGCTTTTAAA AgGAgAATGT AAAACAATTA CTAGTTCTAT ACAgGAgCAT tTAATTAACT

\begin{tabular}{|c|c|c|c|c|c|c|}
\hline$\cdots|\cdots|$ & $\cdots\left|{ }_{7225} \ldots\right|$ & $\cdots\left|{ }_{7235} \ldots\right|$ & $\cdots \mid \begin{array}{l}7245 \\
7 \mid\end{array}$ & $\cdots \mid \begin{array}{l}\cdots 255 \\
721\end{array}$ & $\cdots|\cdots|$ & $\ldots|\ldots|$ \\
\hline$\cdots \cdots$ & $\cdots$ & $\cdots$ & $\cdots$ & $\cdots$ & $\cdots$ & \\
\hline$\cdots$ & - & $\cdots$ & $\cdots$ & $\cdots$ & & \\
\hline$\cdots \cdots \cdots$ & & $\ldots \ldots \ldots$ & 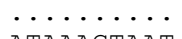 & $\ldots \ldots \ldots$ & & \\
\hline GTGAACACAT & CACTGCAAAA & GAGTTTTCTC & ATAAAGTAAT & CTACACAGTC & AAATGAATTG & GA \\
\hline GTGAACACAT & CACTGCAAAA & GAGTTTTCTC & ATAAAGTAAT & CTACACAGTC & AAATGAATTG & $\mathrm{GAT}$ \\
\hline
\end{tabular}

\begin{tabular}{|c|c|c|c|c|c|c|}
\hline $\begin{array}{c}\cdots|\ldots| \\
7285\end{array}$ & $\begin{array}{c}\ldots|\ldots| \\
7295\end{array}$ & $\begin{array}{c}\cdots|\ldots| \\
7305\end{array}$ & $\begin{array}{c}\ldots|\ldots| \\
7315\end{array}$ & $\begin{array}{c}\ldots|\ldots| \\
7325\end{array}$ & $\begin{array}{c}\ldots|\ldots| \\
7335\end{array}$ & $\begin{array}{c}\ldots|\ldots| \\
7345\end{array}$ \\
\hline$\cdots \cdots$ & $\cdots$ & $\cdots$ & $\cdots \cdots \cdots$ & $\cdots$ & $\cdots$ & \\
\hline$\cdots$ & $\cdots \cdots \cdots$ & $\cdots \cdots \cdots$ & $\cdots \cdots \cdots$ & $\cdots \cdots \cdots$ & $\ldots \ldots \ldots$ & \\
\hline$\dot{\mathrm{TC}}$ & 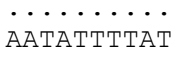 & $\dot{\mathrm{GCA}}$ & $\ddot{\cdots} \cdot \vec{A}$ & $\cdots \vec{A}$ & $\ddot{C G A}$ & \\
\hline
\end{tabular}
AAATTGAATC AAtATTTAA GCATTGCCGg AgCATTGGCA ATCCACGAAC TCAGACCCGA ACTTTGTGTA

\begin{tabular}{|c|c|c|c|c|c|c|}
\hline$\cdots>1 \ldots 1$ & $\cdots>1 \ldots 1$ & $\cdots 1 \ldots 1$ & $\cdots+13 . \cdots 1$ & $\cdots 1 \ldots 1$ & $\cdots \mid \begin{array}{l}7405 \\
\end{array}$ & $\begin{array}{c}\cdots|\ldots| \\
7415\end{array}$ \\
\hline$\cdots$ & $\cdots$ & $\cdots$ & $\cdots$ & $\cdots$ & $\cdots$ & \\
\hline$\cdots$ & $\cdots \cdots$ & $\cdots \cdots \cdot$ & $\cdots \cdots$ & - & - & \\
\hline & . . & - & $\cdots$ & -. & $\cdots$ & \\
\hline $\mathrm{CAC}$ & TGGTCCAACC & ССТСТСАТСС & TTCCTTGCCC & СTGATTCTCT & TTAGTGGTAG & TTCTGA \\
\hline CAGAAACGAC & TGGTCCAACC & ССТСТСАТСС & TTCCTTGCCC & СTGATTCTCT & TTAGTGGTAG & TTCTGATT \\
\hline
\end{tabular}


SmBr 18 scaff000011 EST Contig scaff000068 Contig-0

$\mathrm{SmBr} 18$ scaff000011 EST Contig scaff000068 Contig-0

SmBr18 scaff000011 EST Contig scaff000068 Contig-0

SmBr 18 scaff000011 EST Contig scaff000068 Contig-0

SmBr 18 scaff000011 EST Contig scaff000068 Contig-0

SmBr18 scaff000011 EST Contig scaff000068 Contig-0

SmBr18 scaff000011 EST Contig scaff000068 Contig-0

SmBr18 scaff000011 EST Contig scaff000068 Contig-0

SmBr 18 scaff000011 EST Contig scaff000068 Contig-0

SmBr 18 scaff000011

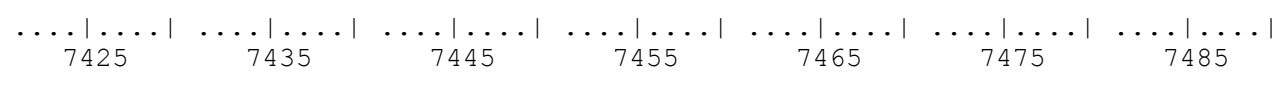

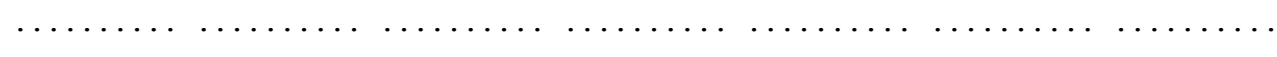

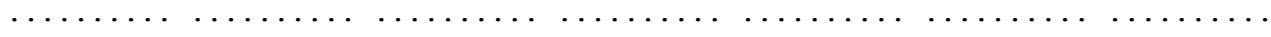

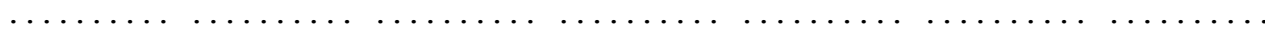

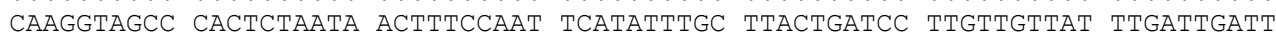

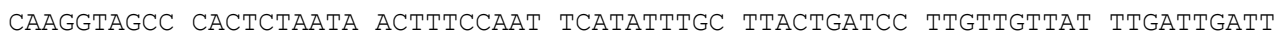

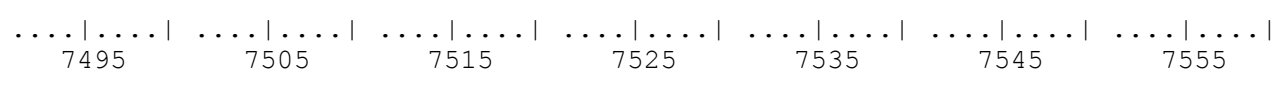

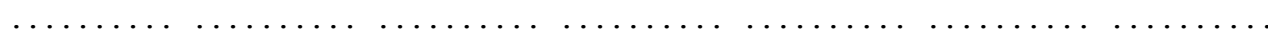

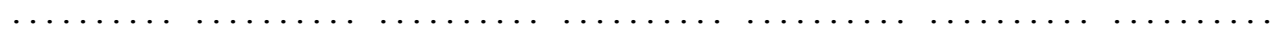

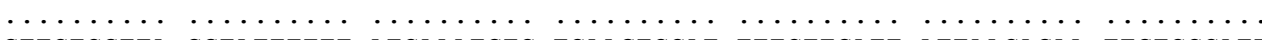
GTTCTCGTTA GGTATTTTTT ATCAAATGTC TCAAGTCCAT TTTCTTGATT ATTAACAGAA TTGTCCCATT GTTCTCGTTA GGTATTTTTT ATCAAATGTC TCAAGTCCAT TTTCTTGATT ATtAACAGAA TTGTCCCATT

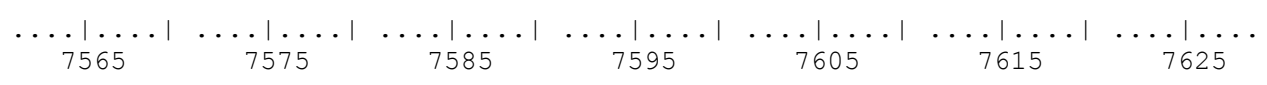

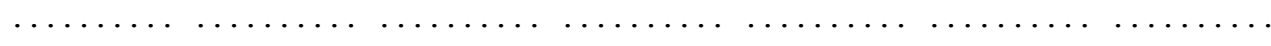

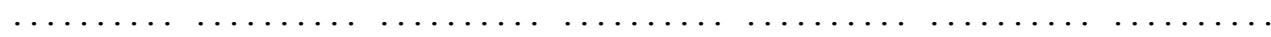

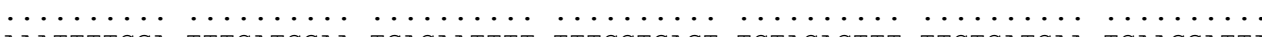
AAATTTTCGA TTTCATCGAA TGACAATTTT TTTCCTGAGT TCTACACTTT TTCTCATCAA TCAAGGATTA

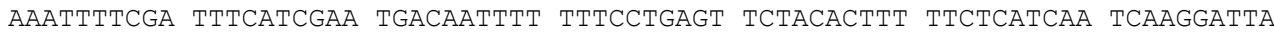

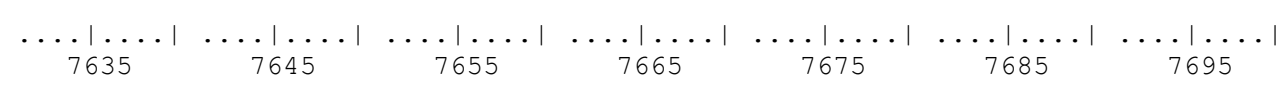

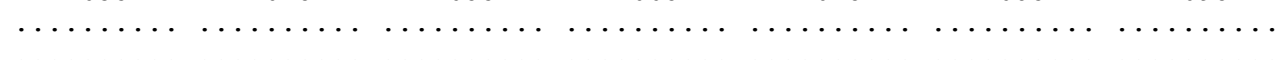

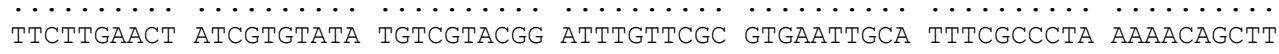

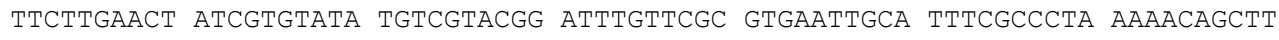

$\ldots|\ldots| \ldots|\ldots| \ldots|\ldots| \ldots|\ldots| \ldots|\ldots| \ldots|\ldots| \ldots|\ldots| \ldots|\ldots| \ldots|\ldots| \ldots \mid$
7705
7715
7725
7735
7745
7755
7765

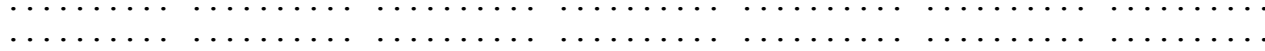
GCTGTCGTTC GTAATTCTTA ATtATCATÄ

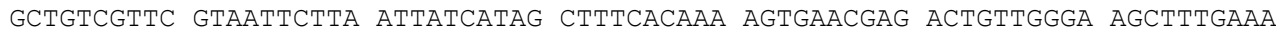

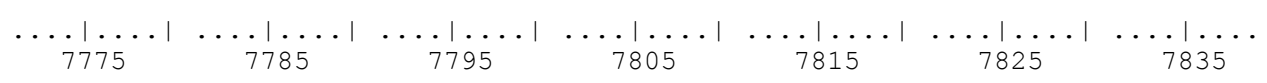

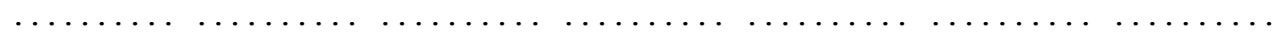

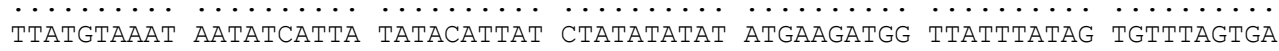

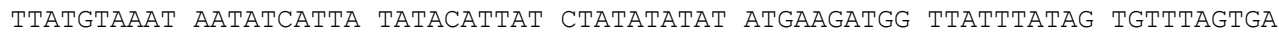

\begin{tabular}{|c|c|c|c|c|c|c|}
\hline$\cdots \mid \begin{array}{l}7845 \\
\end{array}$ & 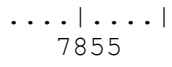 & 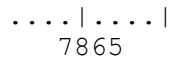 & $\begin{array}{c}\cdots \\
7875\end{array}$ & $\begin{array}{c}\cdots \\
7885\end{array}$ & $\begin{array}{c}\cdots \\
7895\end{array}$ & 7905 \\
\hline$\cdots \cdots$ & $\ldots$. & $\cdots$ & $\cdots \cdots$ & $\cdots$ & $\ldots$ & \\
\hline$\cdots$ & $\cdots \cdots$ & $\cdots \cdots$ & $\cdots \cdots$ & . & & \\
\hline $\begin{array}{c}\text { TTTTTATGTT } \\
\text { TTMA. }\end{array}$ & 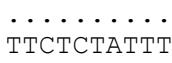 & TTTCСТCTCA & TATCTGTTTA & GATGTTTTCA & ATATTATTGA & $\ddot{\mathrm{TC}}$ \\
\hline TTTTTATGTT & тTстстАттT & тTТССТСТСА & TATCTGTTTA & GATGTTTTCA & ATATTATTGA & $\mathrm{TC}$ \\
\hline
\end{tabular}

\begin{tabular}{|c|c|c|c|c|c|c|}
\hline${ }_{7915}{ }_{71} \ldots \mid$ & 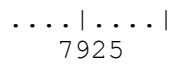 & $\cdots 1 \ldots 1$ & $\begin{array}{c}\cdots|\ldots| \\
7945\end{array}$ & $\cdots \underset{7955}{\cdots}$ & $\cdots+1 \ldots 1$ & $\begin{array}{l}.1 \ldots \\
7975\end{array}$ \\
\hline & & & & & & \\
\hline & . & & & & & \\
\hline & & $\cdots$ & $\cdots$ & $\cdots$ & & \\
\hline TA & TСТСТСАAАA & GAGTTGTTGT & TCAAAA & GCGTAP & $\mathrm{ACCCT}$ & $\mathrm{TT}$ \\
\hline ССТTA & TСТСТСАAАA & GAGTTGTTGT & AATGAC & GCGTAAGTGT & АСССТАТАСт & TTT \\
\hline
\end{tabular}

\begin{tabular}{|c|c|c|c|c|c|c|}
\hline 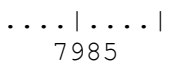 & $\cdots \mid \begin{array}{l}\cdots 995 \\
79 \mid\end{array}$ & $\cdots \mid{ }_{8005}$ & $\cdots|\ldots|$ & $\begin{array}{c}\cdots \mid \ldots 25 \\
801\end{array}$ & $\cdots|\ldots|$ & $\cdots$ \\
\hline$\cdots$ & $\ldots \ldots$ & $\cdots \cdots$ & $\cdots \cdots$ & $\cdots \cdots$ & . & \\
\hline$\cdots$ & $\cdots \cdots$ & . & . & . & & \\
\hline$\ddot{\mathrm{TT}}$ & TTGACTTTCG & GGTCTGATTT & ..... & $\ddot{A G C T G T A C T G}$ & TTTTGTATTG & \\
\hline TATGACTTT & TTGACTTTCG & GGTCTGATTT & TTCACTTGAG & AGCTGTACTG & TTTTGTATTG & TAT \\
\hline
\end{tabular}

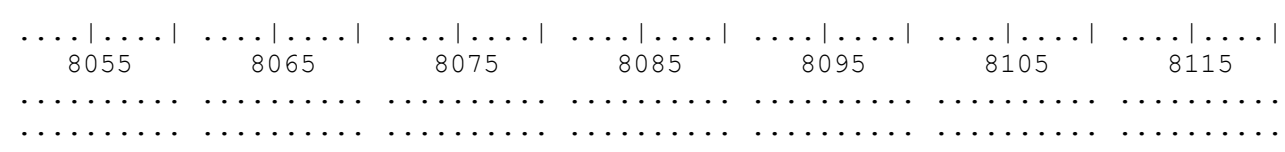


EST Contig scaff000068 Contig-0

SmBr18 scaff000011 EST Contig scaff000068 Contig-0

SmBr18 scaff000011 EST Contig scaff000068 Contig-0

$\operatorname{SmBr} 18$ scaff000011 EST Contig scaff000068 Contig-0

$\mathrm{SmBr} 18$ scaff000011 EST Contig scaff000068 Contig-0

$\mathrm{SmBr} 18$ scaff000011 EST Contig scaff000068 Contig-0

SmBr18 scaff000011 EST Contig scaff000068 Contig-0

SmBr18 scaff000011 EST Contig scaff000068 Contig-0

SmBr18 scaff000011 EST Contig scaff000068 Contig-0

SmBr18 scaff000011 EST Contig scaff000068 contig-0

AAGTTCCCGT TTATAATCCA AAACGTGAAA ACATTTAAAT AAAACACTCG AAAAATAAAT CAAACCTAGT AAGTTCCCGT TTATAATCCA AAACGTGAAA ACATTTAAAT AAAACACTCG AAAAATAAAT CAAACCTAGT

\begin{tabular}{|c|c|c|}
\hline $\begin{array}{l}.|\ldots \ldots| \\
8125\end{array}$ & $\cdots{ }_{8135}$ & $\cdots{ }_{8145}$ \\
\hline
\end{tabular}

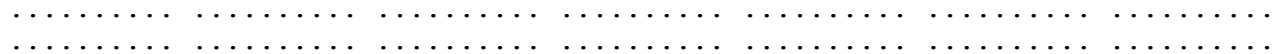
GACCAAATAT GCAATGAAGA CCATTCATAT CACGATTCTG TTCCAGAAAG TAATAAAATC CAGGGTTTTA

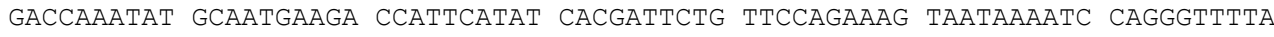

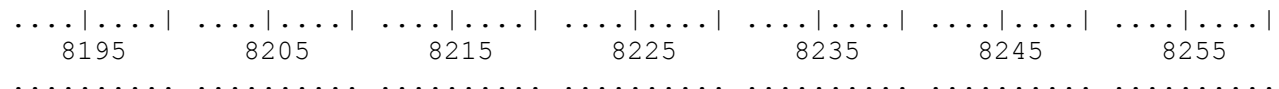

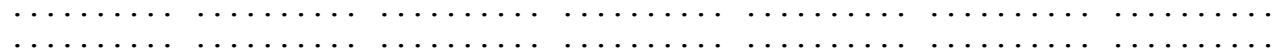
TCACTGCGAA ATGATCAGTA TTGAACCCTT TAAAATCTTT AAAATTATTA GAGTGTTTGA GGTCAGTGTT TCACTGCGAA ATGATCAGTA TTGAACCCTT TAAAATCTTT AAAATTATTA GAGTGTTTGA GGTCAGTGTT

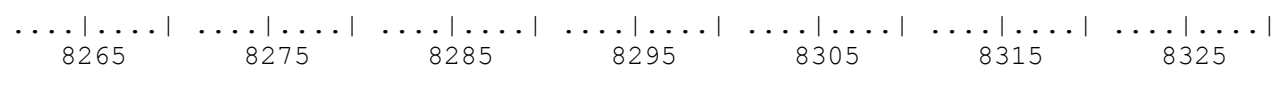

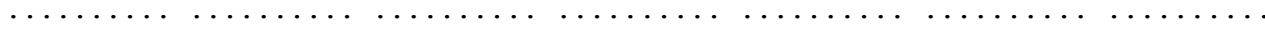
TCAATACGTA TATTTATTCA TGGAAATGTC AтCTACATGA T.

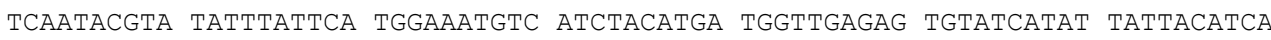

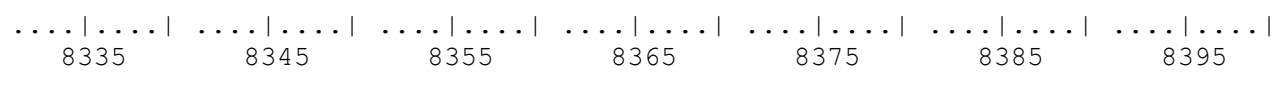
8375

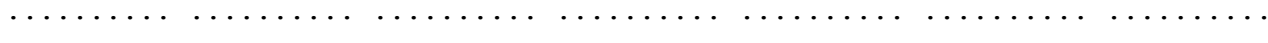

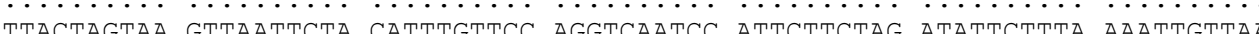
tTACTAGTAA GTTAATTCTA CATTTGTTCC AGGTCAATCC ATTCTTCTAG ATATTCTTTA AAATTGTTAA

\begin{tabular}{|c|c|c|}
\hline$\cdots{ }_{8405}$ & $\begin{array}{c}\cdots|\ldots \ldots| \\
8415\end{array}$ & $\begin{array}{l}. \mid \ldots \\
8425\end{array}$ \\
\hline
\end{tabular}
.

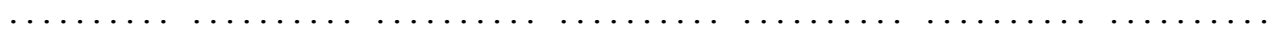

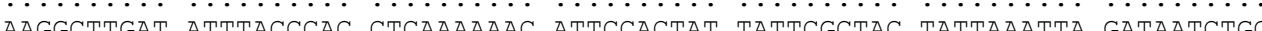
AAGGCTTGAT ATTTACCCAC CTCAAAAAAC ATTCCACTAT TATTCGCTAC TATTAAATTA GATAATCTGC

\begin{tabular}{|c|c|c|}
\hline $\begin{array}{c}\cdots|\ldots| \\
8475\end{array}$ & $\begin{array}{c}\cdots|\ldots| \\
8485\end{array}$ & $\cdots{ }_{8495} \ldots \mid$ \\
\hline
\end{tabular}
.

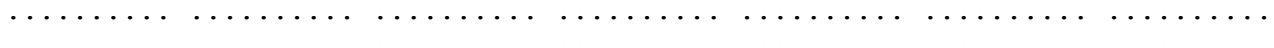
ATCTGCTTTT AGAAAAAGCT ACAGGATTCT GTCACTTCCA TCTATTTAAA CCTTAATTAT TACTAATATC ATCTGCTTTT AGAAAAAGCT ACAGGATTCT GTCACTTCCA TCTATTTAAA CCTTAATTAT TACTAATATC

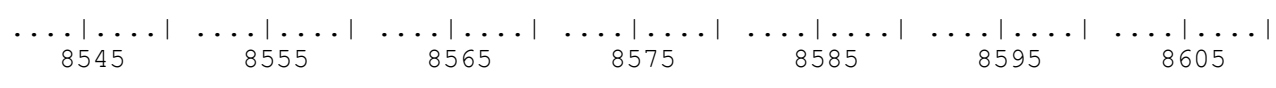

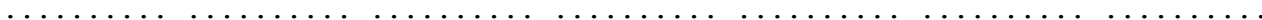

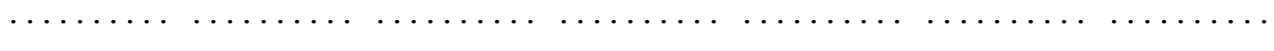
CCTAATATCT TATATGAATT TTTGTGCAAG TTTTCATGTC ATGGACCTAG GCATACAAAA GTTTTATAAT CCTAATATCT TATATGAATT TTTGTGCAAG TTTTCATGTC ATGGACCTAG GCATACAAAA GTTTTATAAT

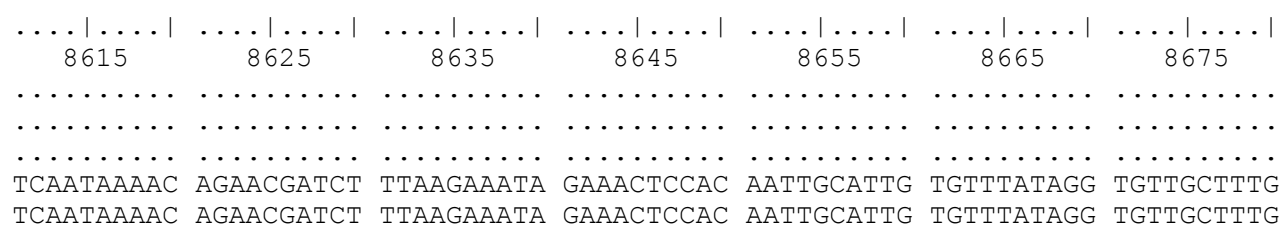

\begin{tabular}{|c|c|c|c|c|c|c|}
\hline$\cdots{ }_{8685}|\ldots|$ & $\cdots{ }_{8695}{ }^{\prime} \ldots \mid$ & $\cdots \underset{8705}{\cdots 1}$ & ${ }_{8715}$ & $\cdots \underset{8725}{\ldots \mid}$ & $\cdots \underset{8735}{\ldots 1}$ & $\cdots \underset{8745}{\cdots}$ \\
\hline & & & & & & \\
\hline & & & & & & \\
\hline CGTTTTGTG & AATTCGTATA & CTACTTGGAT & AGTTTTTTTC & TTCTTCATAG & TATCTTGTGA & $\mathrm{CTTCl}$ \\
\hline CGTTTTGTG & AATTCGTATA & CTACTTGGAT & AGTTTTTTTC & TTCTTCATAG & TATCTTGTGA & СТTCAAT \\
\hline
\end{tabular}




\begin{tabular}{|c|c|c|c|c|c|c|c|}
\hline & $\cdots \mid{ }_{8755}$ & $\cdots{ }_{8765}{ }^{\prime} \mid$ & $\cdots \mid{ }_{8775}$ & ${ }_{8785}$ & $\cdots \mid{ }_{8795}$ & $\cdots|\cdots|$ & 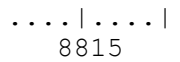 \\
\hline SmBr 18 & $\ldots \ldots \ldots$ & $\ldots \ldots \ldots$ & $\ldots \ldots \ldots$ & $\ldots \ldots \ldots$ & $\ldots \ldots \ldots$ & $\ldots \ldots \ldots$ & $\ldots \ldots$ \\
\hline scaff000011 & $\ldots \ldots \ldots$ & $\ldots \ldots \ldots$ & $\ldots \ldots \ldots$ & $\ldots \ldots \ldots$ & $\ldots \ldots \ldots$ & $\ldots \ldots \ldots$ & $\ldots \ldots$ \\
\hline EST Contig & $\ldots \ldots \ldots$ & $\ldots \ldots \ldots$ & $\ldots \ldots \ldots$ & $\ldots \ldots \ldots$ & $\ldots \ldots$ & $\ldots \ldots \ldots$ & $\ldots \ldots \ldots$ \\
\hline scaff000068 & TGTTATTCCA & AАCTTTTCGG & CATATTGACA & AACATATTTG & AATATAGAGC & СTATTTGGAT & TTATTTTTTA \\
\hline \multirow[t]{2}{*}{ Contig-0 } & TGTTATTCCA & AACTTTTCGG & CATATTGACA & AACATATTTG & AATATAGAGC & CTATTTGGAT & TTATTTTTTA \\
\hline & $\cdots \mid{ }_{8825}$ & $\cdots\left|{ }_{8835}{ }^{\prime}\right|$ & $\cdots\left|{ }_{8845} \ldots\right|$ & $\cdots{ }_{8855}$ & $\cdots\left|{ }_{8865}\right|$ & $\cdots \mid{ }_{8875}$ & $\cdots||_{8885} \ldots \mid$ \\
\hline SmBr18 & $\ldots \ldots \ldots$ & $\ldots \ldots \ldots$ & $\ldots \ldots \ldots$ & $\ldots \ldots \ldots$ & $\ldots \ldots \ldots$ & $\ldots \ldots \ldots$ & $\ldots \ldots \ldots$ \\
\hline scaff000011 & $\ldots \ldots \ldots$ & $\ldots \ldots$ & $\ldots \ldots \ldots$ & $\ldots \ldots \ldots$ & $\ldots \ldots \ldots$ & $\ldots \ldots \ldots$ & $\ldots \ldots \ldots$ \\
\hline EST Contig & $\ldots \ldots \ldots$ & $\ldots \ldots \ldots$ & $\ldots \ldots \ldots$ & $\ldots \ldots \ldots$ & $\ldots \ldots \ldots$ & $\ldots \ldots \ldots$ & $\ldots \ldots \ldots$ \\
\hline scaff000068 & САAАAАAАТА & TAAAAAGTAT & TTGTGTATAT & ATTCCTTGAT & TTGATTTCTT & СTTCCCATAG & ACGTTCTCGA \\
\hline \multirow[t]{2}{*}{ Contig-0 } & CAAAAAAATA & TAAAAAGTAT & TTGTGTATAT & ATTCCTTGAT & TTGATTTCTT & СTTCCCATAG & ACGTTCTCGA \\
\hline & $\cdots \mid{ }_{8895}$ & $\cdots \mid{ }_{8905}$ & $\cdots \mid{ }_{8915}$ & $\cdots{ }_{8925}$ & & & \\
\hline SmBr18 & $\ldots \ldots \ldots$ & $\ldots \ldots \ldots$ & $\ldots \ldots \ldots$ & $\ldots \ldots \ldots$ & & & \\
\hline scaff000011 & $\ldots \ldots \ldots$ & $\ldots \ldots$ & $\ldots \ldots \ldots$ & $\ldots \ldots \ldots$ & & & \\
\hline EST Contig & $\ldots \ldots \ldots$ & $\ldots$ & $\ldots \ldots \ldots$ & $\ldots \ldots \ldots$ & & & \\
\hline scaff000068 & CTGTTAGATT & ATCAACAAGA & ATTGATTAAT & СААСТАAТСА & & & \\
\hline Contig-0 & CTGTTAGATT & ATCAACAAGA & ATTGATTAAT & САACTAATCA & & & \\
\hline
\end{tabular}

Yellow squared letters $=$ CA88 nuclear repetitive DNA sequence end. Squared letters $=$ CA88 nuclear repetitive DNA sequence begin.

Underlined italic $=\mathrm{SmBr} 18$ microsatellite.

Underlined $=$ GT rich region.

In red forward and reverse primers sites. 
TABLE

Predictive variables

Group 1 - Socioeconomic and demographic features

\begin{tabular}{|c|c|}
\hline Variable & Description $^{a}$ \\
\hline HDI-91 & Human development index in year 1991 \\
\hline HDI-00 & Human development index in year 2000 \\
\hline HDII-91 & Income human development index in year 1991 \\
\hline HDII-00 & Income human development index in year 2000 \\
\hline HDIL-91 & Longevity human development index in year 1991 \\
\hline HDIL-00 & Longevity human development index in year 2000 \\
\hline HDIE-91 & Education human development index in year 1991 \\
\hline HDIE-00 & Education human development index in year 2000 \\
\hline Inc without & Families without income $(\%)$ \\
\hline Inc 1 wage & Families with income lower than 1 monthly minimum wage (\%) \\
\hline Inc $1-5$ wages & Families with income from 1-5 monthly minimum wage (\%) \\
\hline Inc $5-10$ wages & Families with income from $5-10$ monthly minimum wage $(\%)^{b}$ \\
\hline Inc $10-15$ wages & Families with income from $10-15$ monthly minimum wage (\%) \\
\hline Inc above 15 & Families with income higher than 15 monthly minimum wage (\%) \\
\hline Ed 1 & Family heads with less than 1 year of instruction or without an education (\%) \\
\hline Ed 1-3 & Family heads with 1-3 study years of literacy level (\%) \\
\hline Ed 4-7 & Family heads with 4-7 study years of literacy level (\%) \\
\hline Ed 8-10 & Family heads with 8-10 study years of literacy level (\%) \\
\hline Ed 11-15 & Family heads with 11-15 study years of literacy level (\%) \\
\hline DAbove15E & Family heads with more than 15 study years of literacy level (\%) \\
\hline EdNonDet 7 & Family heads with non determined study (\%) \\
\hline Urb & Residence in urban area (\%) \\
\hline Rural & Residence in rural area (\%) \\
\hline
\end{tabular}

$a$ : source: SNIU(2005); $b$ : legislation in Brazil establishes a value for a monthly minimum wage, which corresponds nowadays to a value of about U\$200.00.

Group 2 - Basic sanitation

\begin{tabular}{ll}
\hline Variable & Description $^{a}$ \\
\hline WaterPubServ & Residence with access to water supply provided by public service (\%) \\
WaterWells & Residence with access to water supply by means of wells (\%) \\
WaterAnother & Residence with another type of access to water (\%) \\
SewRiverLake & Residence with sewage pumped straight into the sea, rivers or lakes $(\%)$ \\
SewTrench & Residence with sewage connected to trench $(\%)$ \\
SewRudCesspit & Residence with sewage connected to rudimentary cespit (\%) \\
SewSepCesspit & Residence with sewage connected to septic cesspit $(\%)$ \\
Sew & Residence with sewage connected to general network $(\%)$ \\
SewAnother & Residence with another type sewage $(\%)$ \\
WithSan & Residence with toilets $(\%)$ \\
WithoutSan & Residence with no toilets $(\%)$
\end{tabular}

a: source: $\operatorname{SNIU}(2005)$.

Group 3 - Presence of water collections and dense vegetation in the summer

\begin{tabular}{ll}
\hline Variable & Description $^{a}$ \\
\hline BlueS & Blue band in the summer \\
EVIS & Enhanced vegetation index in the summer \\
MirS & Middle infrared band in the summer \\
NDVIS & Normalized difference vegetation index in the summer \\
NirS & Near infrared band in the summer \\
VegS & Linear mixture model - vegetation in the summer \\
RedS & Red band in the summer \\
SoilS & Linear mixture model - soil in the summer \\
ShadeS & Linear mixture model - shade in the summer
\end{tabular}

$a$ : source: MODIS. 
Group 4 - Presence of water collections and dense vegetation in the winter

\begin{tabular}{ll}
\hline Variable & Description $^{a}$ \\
\hline BlueW & Blue band in the winter \\
EVIW & Enhanced vegetation index in the winter \\
MirW & Middle infrared band in the winter \\
NDVIW & Normalized difference vegetation index in the winter \\
NirW & Near infrared band in the winter \\
VegW & Linear mixture model - vegetation in the winter \\
RedW & Red band in the winter \\
SoilW & Linear mixture model - soil in the winter \\
ShadeW & Linear mixture model - shade in the winter \\
\hline
\end{tabular}

a: source: MODIS.

Group 5 - Climate

\begin{tabular}{|c|c|}
\hline Variable & Description $^{a}$ \\
\hline PrecW & Average of accumulated precipitation in the winter \\
\hline PrecS & Average of accumulated precipitation in the summer \\
\hline TmaxW & Average of daily maximum temperature in the winter \\
\hline $\operatorname{TmaxS}$ & Average of daily maximum temperature in the summer \\
\hline TminW & Average of daily minimum temperature in the winter \\
\hline Tmins & Average of daily minimum temperature in the summer \\
\hline \multicolumn{2}{|c|}{$a$ : source: CPTEC/INPE. } \\
\hline \multicolumn{2}{|r|}{ Group 6 - Terrain } \\
\hline Variable & Description $^{a}$ \\
\hline AWater1 & $\begin{array}{l}\text { Average of accumulated water - amount of water that may exist in the mu- } \\
\text { nicipality, calculated from the Dem }\end{array}$ \\
\hline AWater2 & $\begin{array}{l}\text { Median of accumulated water - amount of water that may exist in the mu- } \\
\text { nicipality, calculated from the Dem }\end{array}$ \\
\hline Dem & Digital elevation model of terrain \\
\hline Dec & Slope declivity \\
\hline
\end{tabular}

a: source: SRTM. 


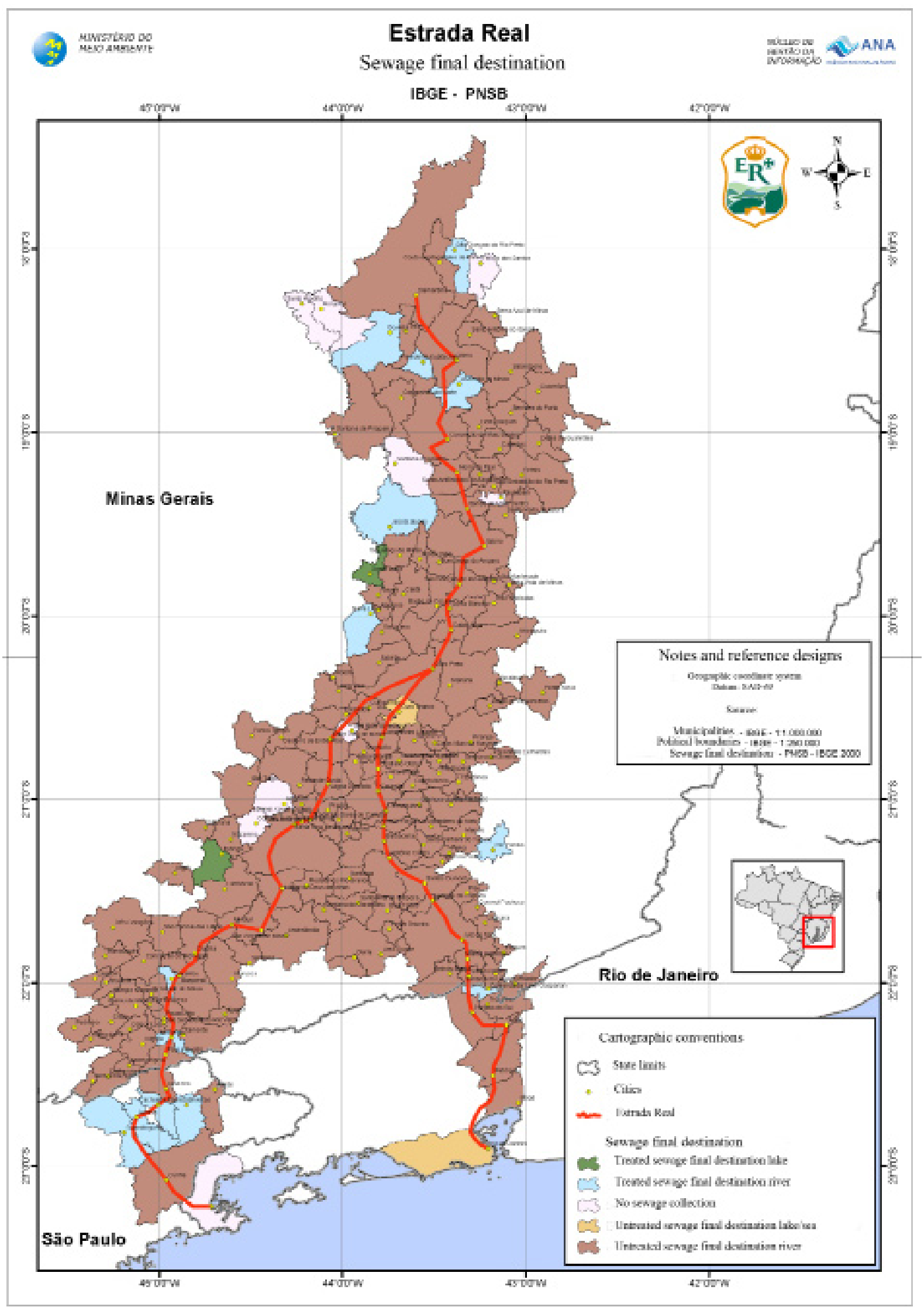

Fig. 1: map showing the municipalities of the Estrada Real project in Minas Gerais, Rio de Janeiro and São Paulo, Brazil and sewage destination (Brazilian Ministry of Environment). 